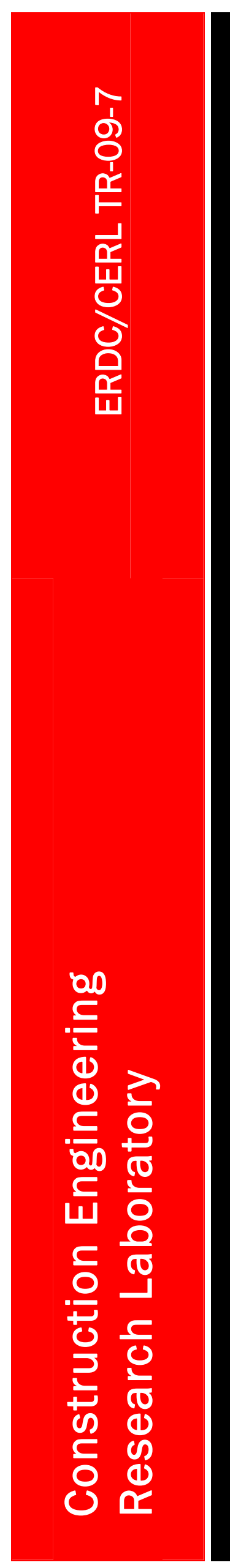

\title{
Gopher Tortoise Survey Handbook
}

Lora S. Smith, Jonathan Stober, Harold E. Balbach, and William D. Meyer

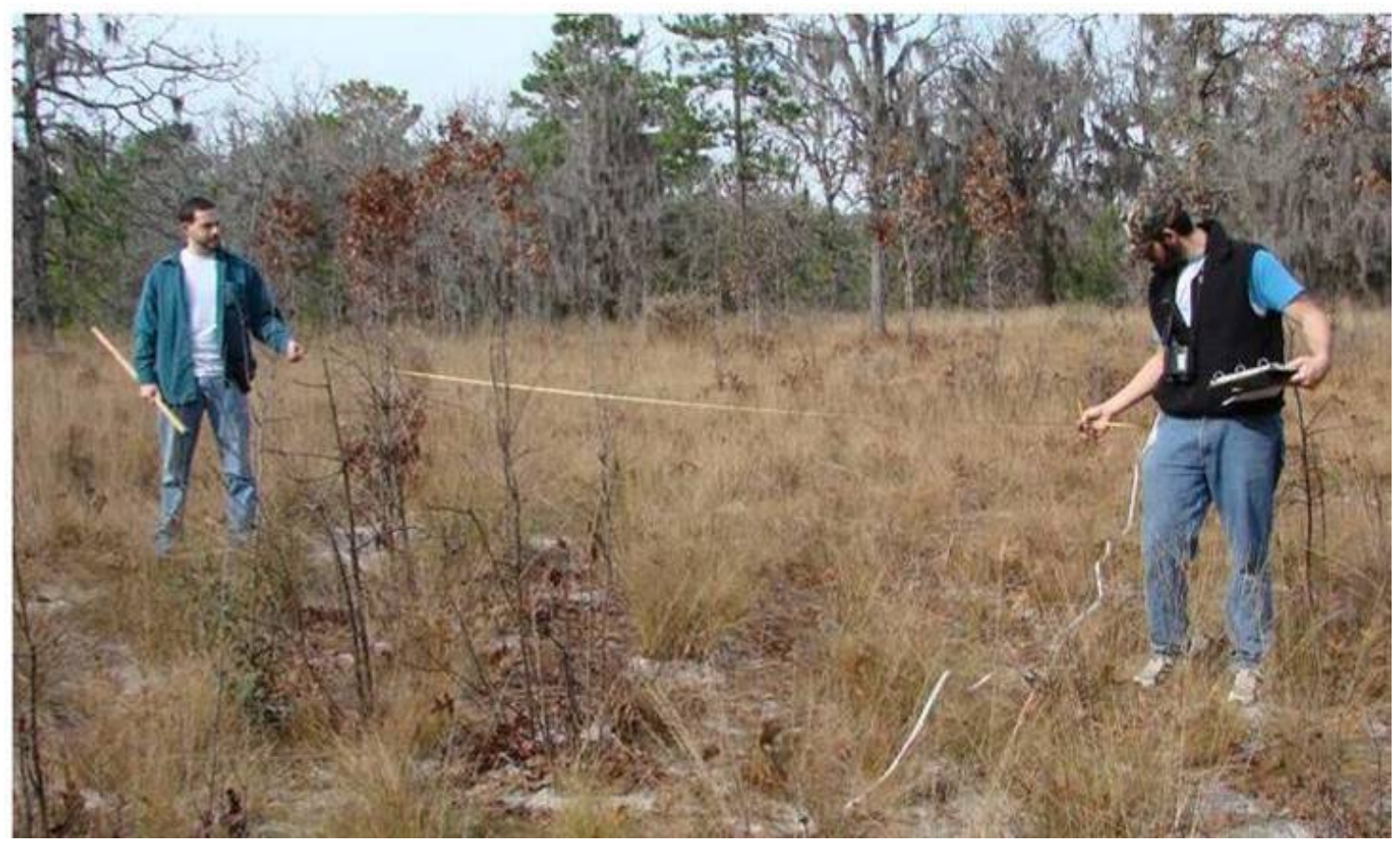





\section{Gopher Tortoise Survey Handbook}

Dr. Harold E. Balbach and William D. Meyer

Construction Engineering Research Laboratory (CERL)

U.S. Army Engineer Research and Development Center

2902 Newmark Dr.

Champaign, IL 61824

Dr. Lora S. Smith, Dr. Jonathan Stober

Jones Ecological Research Center at Ichauway

Route 2 Box 2324

Newton, GA 39870

Final Report

Approved for public release; distribution is unlimited.

Prepared for U.S. Army Corps of Engineers

Washington, DC 20314-1000 


\begin{abstract}
The gopher tortoise (Gopherus polyphemus) has some legal protection, Federal or state, in all of the states where they occur. Threats to the species vary regionally, and it is often desirable to determine in a statistically acceptable manner whether tortoise populations are being maintained or are declining. There is currently no reliable information on the status and trends of populations across the range, although most studies appear to show that declines are almost universal. Gopher tortoise surveys may be conducted for different purposes, e.g., detection of tortoise presence; estimating population size, density, and trends; and temporal monitoring to assess effects of habitat management or restoration. A survey's specific objectives determine its timing and method. Survey and population monitoring methods have not been standardized. This makes it difficult to establish reliable gopher tortoise population information because it is not possible to reliably compare reports among different studies. This handbook outlines standardized methods for gopher tortoise surveys, and suggests which methodologies are best applied to each type of need and habitat setting. The conclusion is that Line Transect Distance Sampling is the most statistically reliable when accurate measurement of tortoise population numbers is needed.
\end{abstract}

DISCLAIMER: The contents of this report are not to be used for advertising, publication, or promotional purposes. Citation of trade names does not constitute an official endorsement or approval of the use of such commercial products. All product names and trademarks cited are the property of their respective owners. The findings of this report are not to be construed as an official Department of the Army position unless so designated by other authorized documents. 


\section{Table of Contents}

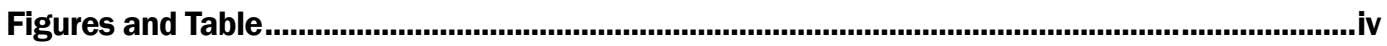

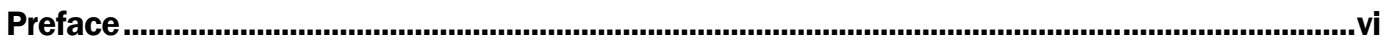

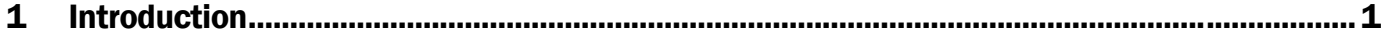

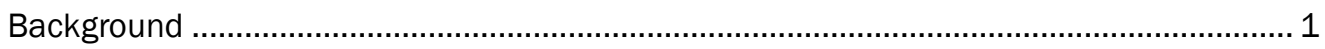

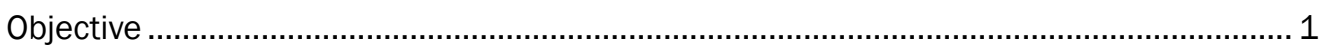

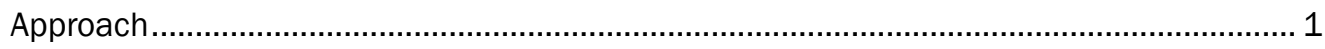

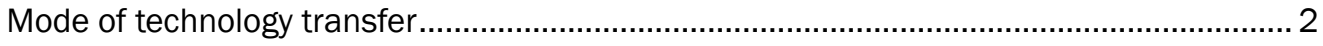

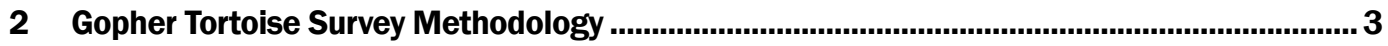

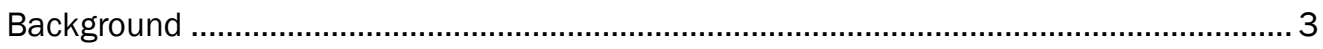

Determination of suitable habitat ............................................................................ 4

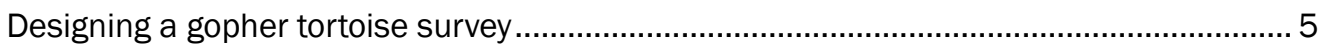

Survey methods - line transect distance sampling........................................................ 8

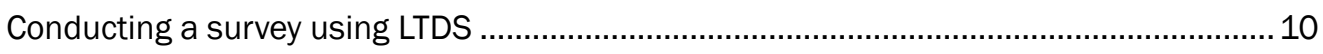

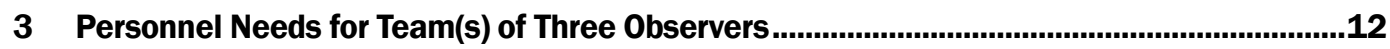

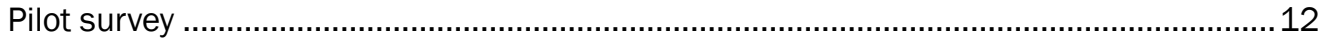

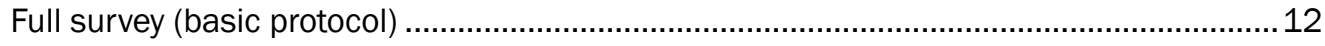

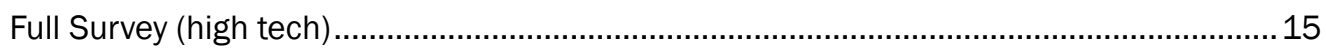

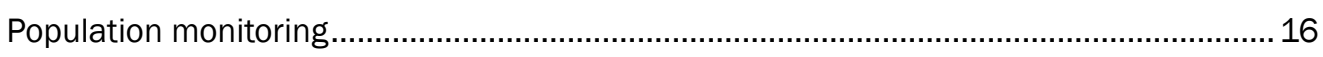

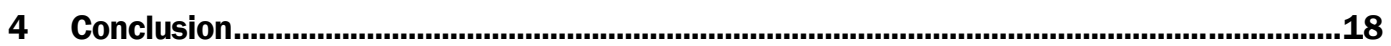

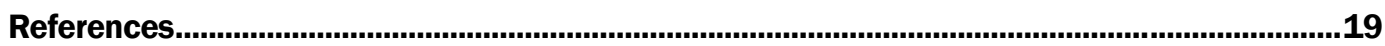

Appendix A: Survey Examples using Line Transect Distance Sampling....................................21

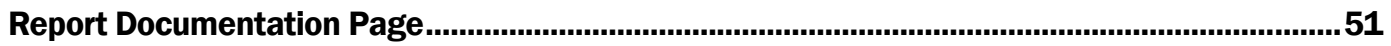




\section{List of Figures and Table}

\section{Figures}

1 Comparison of a gopher tortoise burrow (a) to that of a nine-banded armadillo burrow (b); gopher tortoise burrows are typically half moon shaped and have a mound of soil outside the entrance, whereas armadillo burrows are oval shaped and often occluded by leaf or pine litter

2 Laying out a line transect for distance sampling of gopher tortoises. Equipment needed includes a sighting compass and tape measure.

3 Measuring the perpendicular distance of a gopher tortoise burrow from the transect centerline with a forestry tape measure. Distances of burrows greater than $15 \mathrm{~m}$ from the centerline can be taken with a tape measure.

4 Distance sampling for tortoise burrows along a $100 \mathrm{~m}$ long transect. Distances to burrows (red dots) are measured perpendicular to the transect center line to the mouth of burrows observed (red dots). The assumption is that all burrows on or near the center line are observed. Burrows more distant from the center line may be missed (black dots); however, Program Distance calculates the detection function (an estimate of the burrows missed) based on distance measures of the burrows observed. All burrows are examined with a burrow camera system to determine if they are occupied, and only occupied burrows are used in Distance analysis.

5 Using a camera system to determine whether a burrow is occupied by a gopher tortoise. Observers should be trained in use of the camera system. Unless the operator is certain that they have observed a tortoise, or reached the end of a tortoise burrow, occupancy should be recorded as undetermined

6 Use of calipers to measure the width of a gopher tortoise burrow. Measurements should be taken in metric units $(\mathrm{mm}$ or $\mathrm{cm})$ at $50 \mathrm{~cm}$ inside the burrow entrance........... 15

A1 Sampling frame (in green), which includes the entire site, excluding unsuitable tortoise habitat: flood plain, wetlands, agricultural areas and wildlife food plots .............. 23

A2 Distribution of pilot survey transects ..................................................................................2

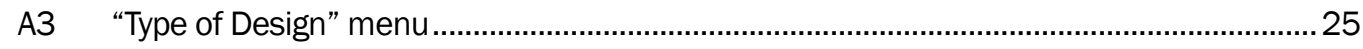

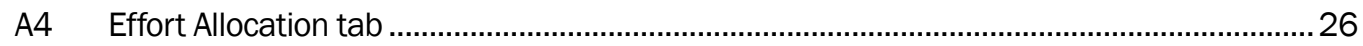

A5 "Sampler" tab of the Design Properties menu ............................................................... 27

A6 “Coverage Probability" tab of the Design Properties menu ................................................ 27

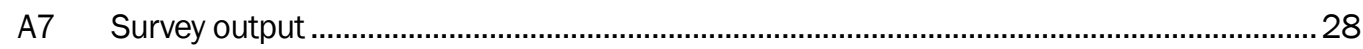

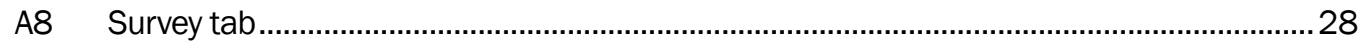

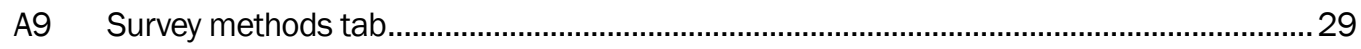

A10 Equal probability sample with $250 \mathrm{~m}$ transects spaced at $700 \mathrm{~m}$ intervals. The eastern terminus of each transect is identified as the starting point..................................30

A11 Sample population example ...................................................................................... 31

A12 Available gopher tortoise habitat and pilot survey design .................................................32

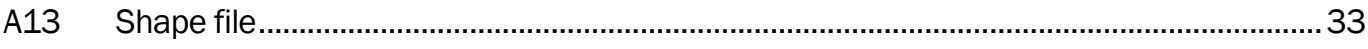

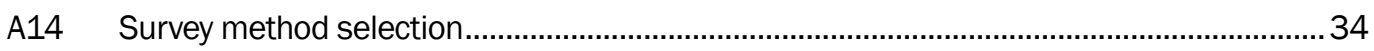


A15 Start and end point coordinates of transect ................................................................

A16 Survey results and map of transects to be surveyed .......................................................35

A17 Distribution of the 21 transects selected within suitable tortoise habitat ...........................36

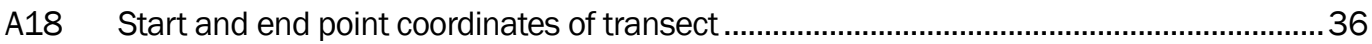

A19 Additional transects placed perpendicular to the previous survey ......................................37

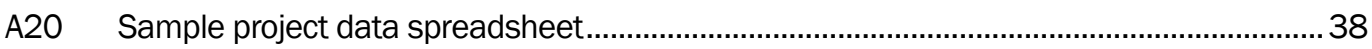

A21 Sample Program Distance template ………….................................................................39

A22 Sample Program Distance data analysis......................................................................... 39

A23 Analysis of individual measured distances data .................................................................. 41

A24 "Intervals" tab - do not click on "transform distance data into intervals for

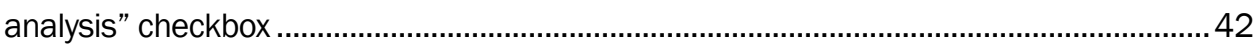

A25 Results of individual measured distances data analysis..................................................42

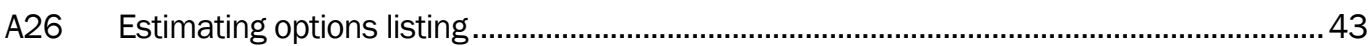

A27 Detection Fct/Golbal/Model output.............................................................................. 43

A28 Graph of fitted probability density function .........................................................................4

A29 Output of Detection Fct/Global/K-S GOF test............................................................ 45

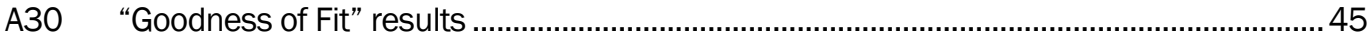

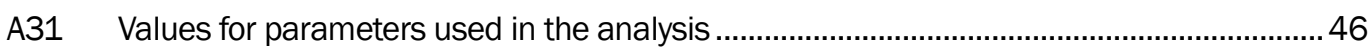

A32 Estimation summary - detection probability tab ............................................................. 46

A33 Estimates for $D$ and $N$ for the area surveyed .................................................................. 47

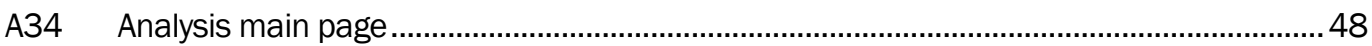

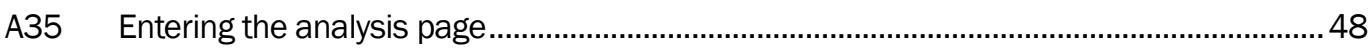

A36 Use the data filter to run one model at a time ................................................................... 49

A37 Acceptable CV (20.76\%) for a small population with a limited sample size ........................ 49

A38 Review the different models on analysis main page ..........................................................50

\section{Table}

1 Metadata associated with standardized tortoise surveys .............................................. 17 


\section{Preface}

This study was conducted for the Army Environmental Quality research program (PE 62720A896) under project number G9K2FL, "GT CCA Support” The technical monitor was Scott Belfit, DAIM-ED.

The work was completed under the direction of the Ecological Process Branch (CN-N) of the Installations Division (CN), Construction Engineering Research Laboratory (CERL). The CERL Principal Investigators and contract monitors was Dr. Harold E. Balbach and William D. Meyer. The handbook development was performed by the Joseph W. Jones Ecological Research Center, Newton, GA. Dr. Lora Smith was the Jones Center Principal Investigator, and Mr. Jonathan Stober is a wildlife biologist at the Center. The study was performed in response to a proposal submitted under the ERDC Broad Agency Announcement, and completed under agreement number W9132T-06-P-0072. Alan B. Anderson is Chief, CN-N, and Dr. John T. Bandy is Chief, CN. The associated Technical Director was Dr. William D. Severinghaus. The Director of CERL is Dr. Ilker Adiguzel.

CERL is an element of the U.S. Army Engineer Research and Development Center (ERDC), U.S. Army Corps of Engineers. The Commander and Executive Director of ERDC is COL Gary E. Johnston, and the Director of ERDC is Dr. James R. Houston. 


\section{Introduction}

\section{Background}

Gopher tortoises are legally protected in the six states where they occur (Smith et al. 2006). Although threats to the species vary regionally, there is a critical need for information on the status and trends of populations across the range. A major factor hindering this goal is the current and historical lack of standardization of survey and population monitoring methods, such that one cannot reliably compare reports among different studies. This handbook outlines standardized methods for gopher tortoise surveys.

A critical first step in determining an appropriate survey methodology is to identify the specific objective(s) of the project. Objectives are often guided, if not defined, by available resources (i.e., funding), size of the area of concern, and other factors such as accessibility to the survey site. However, objectives should ultimately define the development of a sampling strategy and sampling frame for a given tortoise survey. In most cases, gopher tortoise surveys are conducted for one of three purposes:

1. To detect tortoise presence

2. To estimate population size and density

3. To detect trends to assess threats or the effects of habitat management or restoration.

Once identified, the objectives should be used to define both the spatial and temporal scales of the sampling regime.

\section{Objective}

The objective of this work is to present standardized methods for gopher tortoise surveys that will allow users to estimate population size and density and to monitor trends in tortoise populations over time.

\section{Approach}

This handbook outlines the key steps for developing a tortoise population monitoring effort, including how to define suitable habitat (i.e., sampling frame), and criteria for choosing a sampling approach. It also provides standardized methods for estimating tortoise densities and for long-term 
monitoring of the status and trends of populations using Line Transect Distance Sampling (Buckland et al. 2001). Two examples of potential applications of these methods are also provided.

\section{Mode of technology transfer}

The information included in this report is one portion of the materials prepared by the Engineer Research and Development Center to assist installation natural resources and threatened and endangered species program managers. The primary means of communicating the gopher tortoise health manual information will be through publication in the scientific literature, as well as through the availability of this report.

This report will be made accessible through the World Wide Web (WWW) at URL: http://www.cecer.army.mil 


\section{Gopher Tortoise Survey Methodology}

\section{Background}

Gopher tortoise survey methods have traditionally focused on counting burrows in suitable habitat using sampling plots (quadrants or strip transects) or total burrow counts to derive estimates of tortoise density (Cox et al. 1987; Carthy et al. 2005). These methods did not estimate detection probability or "detectability"; they assumed that all burrows were observed in the survey. This assumption is rarely met in wildlife surveys, and can be a serious source of error (Thompson et al. 1998; Pollock et al. 2004). A measure of detectability must be incorporated into survey designs when population density estimation is the chosen objective. Additionally, because gopher tortoise burrow occupancy can vary significantly among populations (Burke 1989; McCoy and Mushinsky 1992; Smith et al. 2004), neither burrow density nor burrow activity status are suitable surrogates for tortoise density, especially when different sites must be compared.

Gopher tortoises excavate and use multiple burrows within and between seasons; therefore, counting burrows overestimates the number of tortoises present on a site, and produces biased results. Accurate tortoise population estimates can only be obtained by examining each burrow using a burrow camera system (Smith et al. 2004) to determine whether burrows are occupied by a tortoise at the time of the survey. Rather than using a burrow camera, the traditional method of assessing whether a gopher tortoise burrow was occupied was based on the "activity status" of the burrow (Auffenberg and Franz 1982).

The activity assessment of burrows is highly subjective and is unreliable for population estimation (Smith et al. 2004). Many surveys also used a standard burrow occupancy estimate, often referred to as a "correction factor" to convert the number of burrows into the number of tortoises. The standard occupancy estimate that has been widely used for more than 25 years (61.4 percent of active and inactive burrows) was based on work done by Auffenberg and Franz (1982) on a long-term study site. These data were never intended to be broadly applied and numerous studies have since demonstrated that burrow occupancy can vary from 20 percent to more than 90 percent at different sites (Burke 1989; Breininger et al. 
1991; McCoy and Mushinsky 1992, Smith et al. 2004; Witz et al. 1991; R. Ashton, pers. comm.).

More recently, a value of 50 percent occupancy for all burrows regardless of activity status has been proposed (Ashton and Ashton, in press). However, this figure was derived by averaging occupancy values from different sites and thus likely represents either an over- or under-estimate of the actual burrow occupancy at any given site. The use of burrow activity status as an indicator of tortoise numbers and reliance on a standard burrow occupancy estimate have resulted in biased, inaccurate, and questionable data on the status and trends of tortoise populations across the range. Furthermore, it is not possible to assess the status and trends of gopher tortoise populations across its range without standardization of survey methods at each population. Accurate gopher tortoise surveys require direct observations of animals, rather than burrows. This will clearly require more effort than simply counting burrows. However, the additional effort will derive statistically reproducible data that may be compared across time and across different sites, and that can be presented to managers and regulatory officials with confidence.

This handbook outlines the key steps for developing a tortoise population monitoring effort, including how to define suitable habitat (i.e., sampling frame) and criteria for choosing a sampling approach. It also provides standardized methods for estimating tortoise densities and for long-term monitoring of the status and trends of populations using Line Transect Distance Sampling (Buckland et al. 2001). Appendix A to this report provides two examples of potential applications of these methods. The ultimate goal is to provide efficient and statistically robust survey methods that will yield comparable data for populations across the range of the species.

\section{Determination of suitable habitat}

Gopher tortoises occur in a wide range of natural communities as well as in many disturbed or anthropogenic habitats. Therefore, vegetation alone is not the most reliable indicator of gopher tortoise presence. Although diverse herbaceous ground cover and an open canopy are important components of tortoise habitat, soil type is the single most reliable indicator of suitable habitat. Gopher tortoises require deep, sandy, well-drained soils for burrowing. However, they may also occur in lower numbers on somewhat poorly drained soils, such as those of pine flatwoods communities. Soil surveys have been published by the Natural Resource Conservation 
Service for many regions and can provide fine-scale detail on soil types (http://soils.usda.gov/). Mississippi has designated priority soil types for tortoise surveys. In other states, priority tortoise habitat is designated by natural communities (Florida Natural Areas Inventory, Guide to the Natural Communities of Florida: http://www.fnai.org/PDF/Natural_Communities_Guide.pdf).

If the goal is to estimate population size and/or to monitor a population through time, all areas with suitable soils should be surveyed. Tortoises can persist in small patches of suitable vegetation along roadsides, edges of retention ponds, power line right-of-ways, tree fall gaps, and old fields; therefore, in appropriate soils, these areas should also be included in a sampling frame. In defining suitable tortoise habitat for surveys, a composite of factors should be considered including soil type, vegetation, landuse, and fire history. This should be done with the recognition that this is the basis from which a statistical inference will be made for the population of interest. Wetland and floodplain soils should be excluded because they generally represent unsuitable habitat; however, they can be stratified separately if surveys of these habitats are required. The sample area should include only actual or potential tortoise habitat, since sampling in areas not suitable for tortoises will skew the resulting density measures inappropriately. This can be relatively complex if the area is not uniform in terms of soils or terrain, and will require an assessment of both soils and vegetative cover.

Once the survey objectives and potential habitats are identified, a sampling approach for the population of interest can be developed. In some situations, it may be necessary to stratify the sample to determine tortoise density in habitats that are potentially very different (e.g., sandhill and flatwoods).

\section{Designing a gopher tortoise survey}

Line transect distance sampling (LTDS, Buckland et al. 2001) is the recommended method for gopher tortoise surveys. Distance sampling is efficient and can provide robust, unbiased estimates of tortoise density (Averill-Murray and Averill-Murray 2005; Anderson et al. 2001; Carthy et al. 2005; Krzysik 2002; Swann et al. 2002). The open source software program Distance 5.0 (http://www.ruwpa.st-and.ac.uk/distance/, Thomas et al. 2003) can be used to create survey designs and to analyze data in a variety of ways. Line transect distance sampling can be used to sample both high and low density populations and is easy to employ at large scales. 
The first step in designing a survey using LTDS is to define the specific habitat or ecosystem to be sampled, called the "sampling universe." For example, the Department of Defense may wish to know the tortoise population density on a particular military installation; hence, the sampling universe is the entire military installation. The second step is defining the area within the sampling universe to be surveyed, called the "sampling frame" (e.g., a GIS map with suitable habitat delineated or the population of desired inference). The third step is distributing a sample across the sampling frame. To avoid bias, the sample should be randomly selected or placed within all available suitable habitat.

Once a potential sampling frame is defined, a Geographic Information System (GIS) can be used to create a shape file of it. Next, a pilot survey must be conducted to determine the tortoise encounter rate (Buckland et al. 2001, p 240), i.e., the number of tortoises observed along a length of transect. While conducting the pilot survey, the sampling frame should be evaluated to determine if it accurately delineates suitable tortoise habitat; if not, it can be adjusted accordingly. The pilot survey data will be used to determine the sampling intensity needed for the full survey.

However, a pilot survey can also be used to verify tortoise presence/absence (if this is not known), or to determine the distribution of tortoises across a sampling frame (see below). For surveys to estimate population density, observation of $10-15$ tortoises is usually sufficient to determine the encounter rate (see Appendix A). The encounter rate can then be used to calculate the total length of transect necessary to detect at least $60-80$ tortoises as recommended by Buckland et al. (2004), and to derive estimates with the desired confidence intervals and coefficient of variation (Buckland et al. 2001, page 242). Although Buckland et al. (2001) recommend observation of at least 60-80 tortoises across all transects to estimate population size, in some cases, smaller sample sizes (40 or less) may be adequate (Buckland et al. 2004). The number of transects needed and the expected variation of the estimate (CV) can be determined based on past estimates of population density, but a pilot survey is strongly recommended if possible.

In homogeneous habitat, an "equal probability" sample can be used to estimate tortoise abundance. In an equal probability sample, all available habitat has an equal probability of being included and all habitat is sampled with equal intensity. Equal probability sampling avoids complicated weighted analysis and is the recommended technique. In heterogeneous 
sites, consider using systematic stratified sampling (e.g., by habitat type) to minimize within-stratum variability. All of these decisions should be made in consultation with someone familiar with distance sampling as well as the objectives of the survey and conditions of the area to be surveyed.

In large areas of contiguous habitat, either randomly spaced parallel transects or systematic random transects can be used. In a systematic random survey, transects are spaced parallel to each other at fixed distances apart. In a systematic sample, the beginning of the first transect is randomly placed satisfying the statistical condition for a simple random sample. For larger parcels of habitat (>500oha), a systematic segmented grid or a systematic segmented trackline sampling could be used to disperse the sample over a large area. A systematic random survey is often the preferred method for most situations. Transects should be oriented perpendicular to topographic contours and parallel to any known gradient, if possible. The transect distribution, length, orientation, start and end points, and data analysis can be developed using GIS shape files and DISTANCE 5.0 software (See Example 1 in Appendix A to this report [p 21]).

For extremely small tortoise populations (e.g., 15-30 animals) it will be necessary to use "repeated surveys" to obtain sufficient observations of tortoises. For repeated surveys, transects can be sampled multiple times and the data can be pooled to derive an abundance estimate (See Example 2 in Appendix A to this report [p 30]). In some circumstances, e.g., when there are fewer than 10 tortoises on a small tract of land $(<200 \mathrm{ha})$, an intensive total survey should be considered. In a total survey, parallel, adjacent transects can be sampled such that all the suitable habitat within the site is covered using LTDS methods. If the site is large (>100oha), but has very few tortoises $(<20)$, at least 15 percent of suitable habitat should be surveyed using LTDS methods outlined below. If basic distance data is collected on sites with small populations or very low population densities, the data can serve as a pilot survey, or in some situations the data can be pooled over time to derive density estimates. When a total survey is conducted on small populations ( $<10$ tortoises), a second, independent observer or team of observers should be used to estimate detectability (Nichols et al. 2000). Program Distance can also be used for analysis of Double observer data.

As a general rule, to detect changes in population size over time, sampling should be intensive enough to produce a coefficient of variation (CV) that 
is approximately $\leq 20$ percent. A CV of 15 to 20 percent is a practical expectation for most monitoring projects. Attaining a lower CV (e.g., 10 percent) would be prohibitively costly in terms of labor/effort. In contrast, once the $\mathrm{CV}$ exceeds 20 percent, the statistical power and confidence and ability to detect trends in monitoring data is substantially reduced.

For long-term objectives, define the measure of success in advance, e.g., increased tortoise density or recruitment (increased number of hatchling or juvenile burrows). To monitor population trends, the same transects should be re-sampled over time. The gopher tortoise is a long-lived species and demographic changes typically occur over long time periods. Therefore, after an initial baseline survey is conducted, surveys should be repeated at regular intervals (e.g., every $3-5$ years) to monitor long-term population trends. It is important to remember that population trends can only be determined when methods are standardized over time and when estimates include a measure of detectability and confidence intervals.

\section{Survey methods - line transect distance sampling}

Five basic assumptions of LTDS, must be met for accurate population density estimates:

1. All burrows and tortoises directly on the transect center-line are found.

2. Objects are detected at their initial location, prior to any response to the observer (not an issue since a large proportion of the time the burrow entrance is used as a tortoise location).

3. Transect length is measured accurately.

4. Perpendicular distance from the transect to the burrow is measured accurately.

5. The transects themselves, or systematic placement of the transects is random.

Since surveys will be focused on observations of occupied burrows, the location of the burrow entrance will serve as the location of the individual. Any tortoises observed on the surface will be recorded in the same manner as a burrow.

Gopher tortoise burrows can be identified by the characteristic half moon shape (the shape of a tortoise shell in profile) and the mound of soil at the burrow entrance. Burrows of nine-banded armadillo can be mistaken for gopher tortoise burrows, and armadillos can sometimes modify and use tortoise burrows. However, armadillo burrows are oval in shape, are gen- 
erally $<1.5 \mathrm{~m}$ long, and do not typically have a large mound of soil at the entrance (Figure 1). During the active season for tortoises (generally from March-October), the soil at the burrow entrance is often freshly turned and tortoise tracks may be present. However, tortoises may abandon burrows and unoccupied burrows can persist for some time. Therefore, it is critical that tortoise burrows be examined with a camera to confirm tortoise presence rather than to assess presence on subjective features, such as tracks.

It is also critical that field personnel receive training in the use of a burrow camera system and identification of occupied burrows. Even experienced observers cannot always determine whether a burrow is occupied (see the Example in Appendix A to this report [p 21]) and it is important to report the percentage of burrows for which occupancy could not be determined. In addition, the burrow cameras that are commercially available are $>5.8 \mathrm{~cm}$ in diameter, which is too large to scope hatchling and small juvenile burrows. Therefore, surveys with these camera systems provide an estimate of the adult (reproductive) population and a portion of the juvenile population. If the objective is to estimate juvenile densities, a smaller 2.5-cm diameter camera can be made or purchased to survey small burrows.

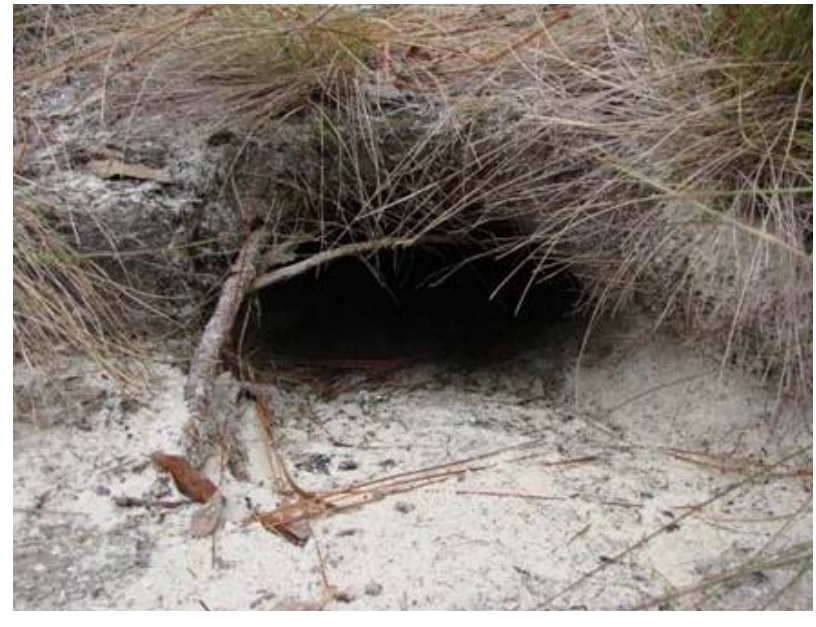

a.

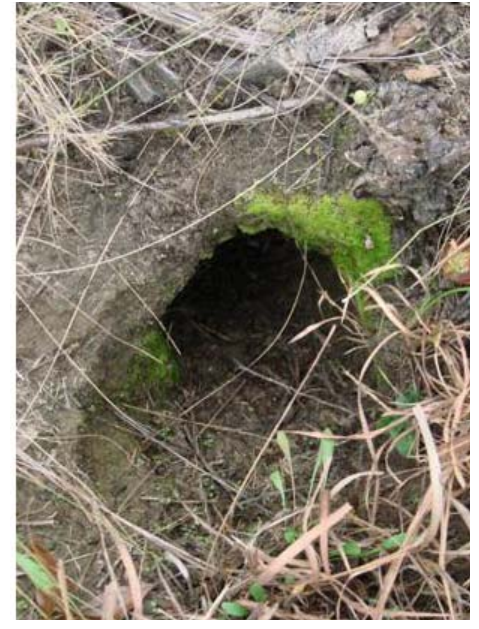

b.

Figure 1. Comparison of a gopher tortoise burrow (a) to that of a nine-banded armadillo burrow (b); gopher tortoise burrows are typically half moon shaped and have a mound of soil outside the entrance, whereas armadillo burrows are oval shaped and often occluded by leaf or pine litter. 
If the objective of a survey is simply to confirm tortoise occurrence on a site, all burrows encountered can be examined with a camera until a tortoise is observed, after which no more sampling is required. For some purposes, this simple confirmation of presence may initially be adequate for preliminary planning actions. This informal method would require significantly less effort than estimating population size, and if the length of transect and number of tortoises is recorded, the information could later be used as a pilot study using LTDS (see below). Negative evidence from surveys (i.e., not finding burrows or live tortoises) cannot be interpreted as confirmation that tortoises are absent from a site. This conclusion can only be reached if the survey has adequate statistical power (Bausell and $\mathrm{Li}$ 2002).

\section{Conducting a survey using LTDS}

The protocol outlined in this section can be applied to most tortoise surveys using line transect distance sampling, and can be adapted to different situations depending on the objectives and scale of a project, as well as to the available resources. However, all effort should be made to incorporate factors mentioned in the previous section and to meet the basic assumptions of the protocol. Two different protocols are outlined below, one that relies on basic field equipment (a compass and measuring tape) and another that relies on newer, field computer/GPS technology.

The tradeoffs between the basic and high tech protocols are as follows: the basic protocol requires less initial investment in equipment, but requires more time in the field and for data management. The more technological approach requires an initial investment in a field computer/GPS (approximately $\$ 4000$ at current prices), and additional training for the survey crew, but is much more efficient (e.g., data are handled electronically, and no compass or tape measure is needed). Used correctly, the field computer/GPS is more precise and efficient than using a compass and tape measure.

The following equipment will be needed:

- compass

- tape measure (100m)

- forestry tape (loggers tape)

- flagging, pin flags

- burrow calipers 
- Global Positioning System (GPS)*

- ArcGIS and ArcPAD or similar software

- distance 5.0 software (http://www.ruwpa.st-and.ac.uk/distance/)

- burrow camera (5-6m scope length) ${ }^{\dagger}$

- datasheets (see Appendix A)

- flexible conduit (2-3m) extension for burrows deeper than $5 \mathrm{~m}$ (attach with duct tape).

*The use of a GPS with real-time submeter accuracy and field computer (e.g., Trimble Nomad) is recommended. This system requires ArcGIS and ArCPAD, or similar software.

† Burrow camera systems are commercially available for $\$ 2000-\$ 6000$ (e.g., Sandpiper Technologies, Inc. and Fuhrman Diversified, Inc.), or can be custom made. Equipment can also be rented (http://www.sandpipertech.com/rentals.html). Sandpiper Technologies offers a $2.5 \mathrm{~cm}$ diameter camera that can be used to search very small burrows. However, the smaller diameter cameras do not have sufficient range of view for use in adult burrows. 


\section{Personnel Needs for Team(s) of Three Observers}

\section{Pilot survey}

The first step in using Line Transect Distance Sampling is to conduct a pilot survey to determine the tortoise encounter rate (the number of animals observed per total transect length sampled). As a general rule, pilot surveys should cover the range of suitable tortoise habitats on a site, and must yield from $5^{-15}$ observations of tortoises (10-15 preferred) to develop a full survey. The pilot survey should also be used to verify the delineation of suitable tortoise habitat that will ultimately define the sample frame for the survey. Pilot survey transects can be of variable length, and surveys simply require recording observations of tortoises either above ground or in burrows (using a burrow camera system). The encounter rate is then used to extrapolate the total length of transect necessary to develop a full survey to estimate tortoise density with the degree of precision (CV) outlined in the objectives. The following examples give details.

\section{Full survey (basic protocol)}

The Geographic Information System (GIS) shape file of the sampling frame should be imported into DISTANCE 5.0 software and the transect distribution, length, orientation, start and end points, and data analysis can be developed (see Appendix A). Transect starting points generated in Distance can be reformatted as XY coordinates in Notepad and uploaded into a Global Positioning System (GPS) as waypoints. The field survey team can then navigate to the starting point of each transect with the GPS.

At each transect starting point, a tape measure should be laid out in a preselected compass heading for the transect (Figure 2); the tape measure will serve as the transect centerline. One person should search the centerline and the area close to the centerline for burrows. Two additional observers should search on either side of the centerline weaving back and forth to detect burrows from the centerline outward. Transect width can be truncated (Buckland et al. 2001; p 15) so that the search effort is contained within a fixed perpendicular distance (e.g., 20 or 30m) of the centerline to increase sampling efficiency. However truncating transect width in the field should only be considered when sample sizes are sufficient. 


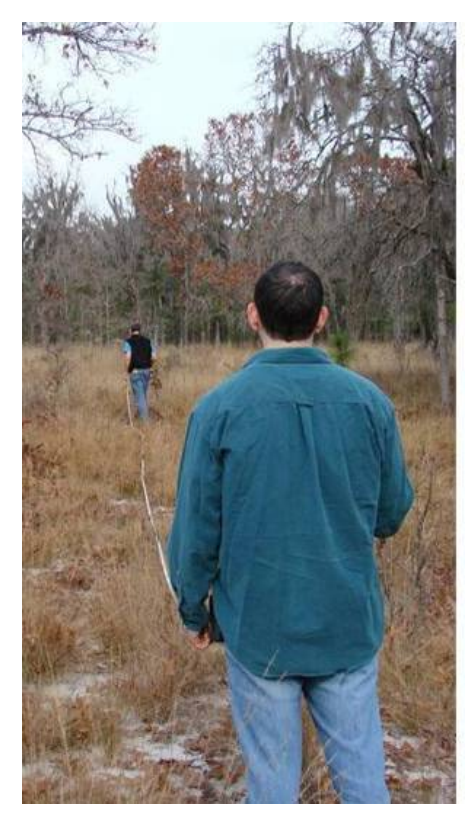

Figure 2. Laying out a line transect for distance sampling of gopher tortoises. Equipment needed includes a sighting compass and tape measure.

When a burrow is observed, the perpendicular distance measurement from the transect centerline to the burrow opening $( \pm 1.0 \mathrm{~m})$ should be taken with either a logger's tape or measuring tape (Figures 3 and 4). All burrows should then be marked with a flag to prevent inadvertently counting the same burrow twice.

Immediately after surveying a transect, or at regular intervals (e.g., every $250 \mathrm{~m}$ ), observers should return to the flagged burrows to search for tortoises with a burrow camera (Figure 5). Observers must be trained in use of the camera system because, even with a camera, it can be difficult to determine with certainty whether a tortoise is present in a burrow (Smith et. al. 2004). Burrows can be too long for the camera scope, or it may be impossible to navigate the camera around curves or obstructions in the burrow. If the burrow is deeper than the 5-6 $\mathrm{m}$ probe, an additional $2-3 \mathrm{~m}$ of 3/4-in. diameter PVC or Pix conduit can be duct-taped to the probe and to extend its length to 8-9 $\mathrm{m}$. All burrows that are scoped should be categorized as either:

1. Tortoise observed,

2. No tortoise observed for entire length of burrow, or

3. Unable to determine if occupied.

The width of all burrows should be measured $50 \mathrm{~cm}$ inside the opening using burrow calipers (Figure 6) to provide information on the demography of population (Alford 1980; and Martin and Layne 1987). 


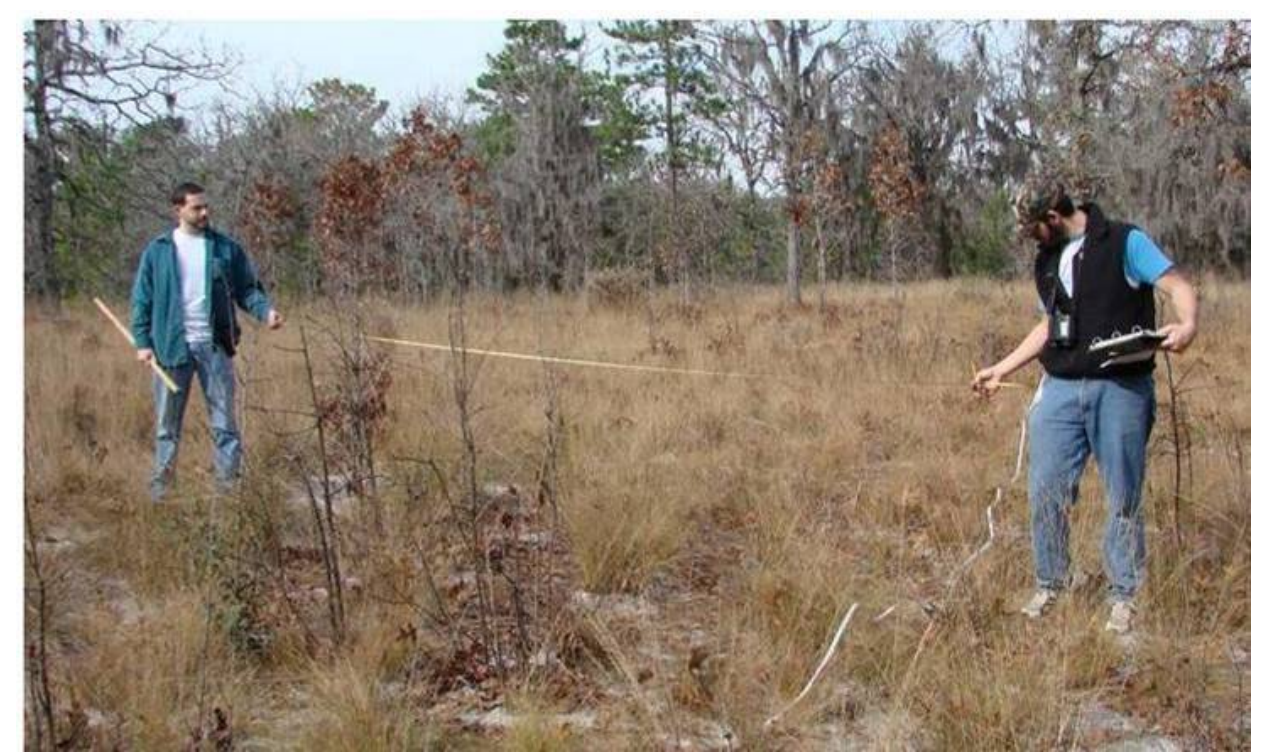

Figure 3. Measuring the perpendicular distance of a gopher tortoise burrow from the transect centerline with a forestry tape measure. Distances of burrows greater than $15 \mathrm{~m}$ from the centerline can be taken with a tape measure.

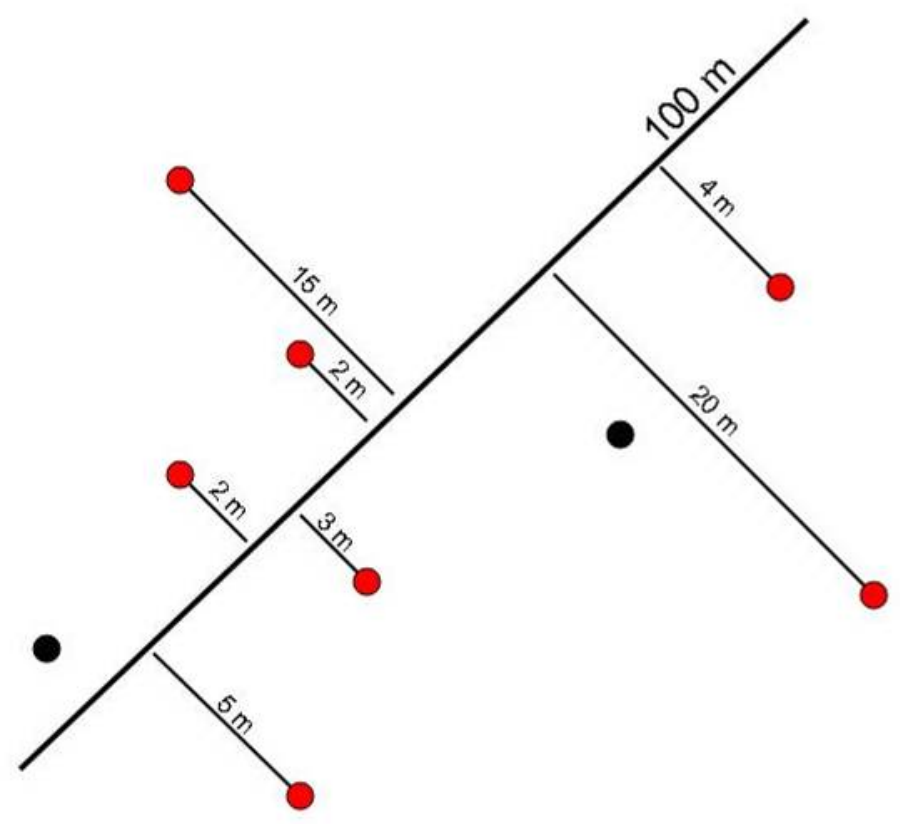

Figure 4. Distance sampling for tortoise burrows along a $100 \mathrm{~m}$ long transect. Distances to burrows (red dots) are measured perpendicular to the transect center line to the mouth of burrows observed (red dots). The assumption is that all burrows on or near the center line are observed. Burrows more distant from the center line may be missed (black dots); however, Program Distance calculates the detection function (an estimate of the burrows missed) based on distance measures of the burrows observed. All burrows are examined with a burrow camera system to determine if they are occupied, and only occupied burrows are used in Distance analysis. 

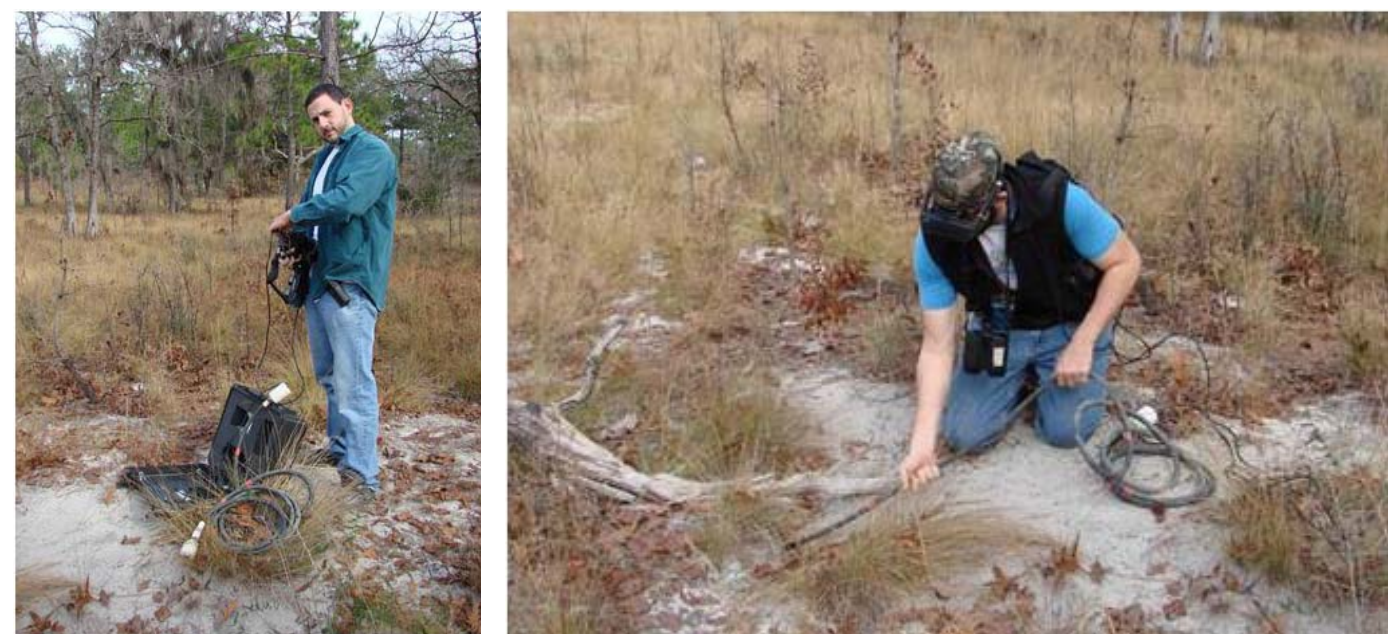

Figure 5. Using a camera system to determine whether a burrow is occupied by a gopher tortoise. Observers should be trained in use of the camera system. Unless the operator is certain that they have observed a tortoise, or reached the end of a tortoise burrow, occupancy should be recorded as undetermined.
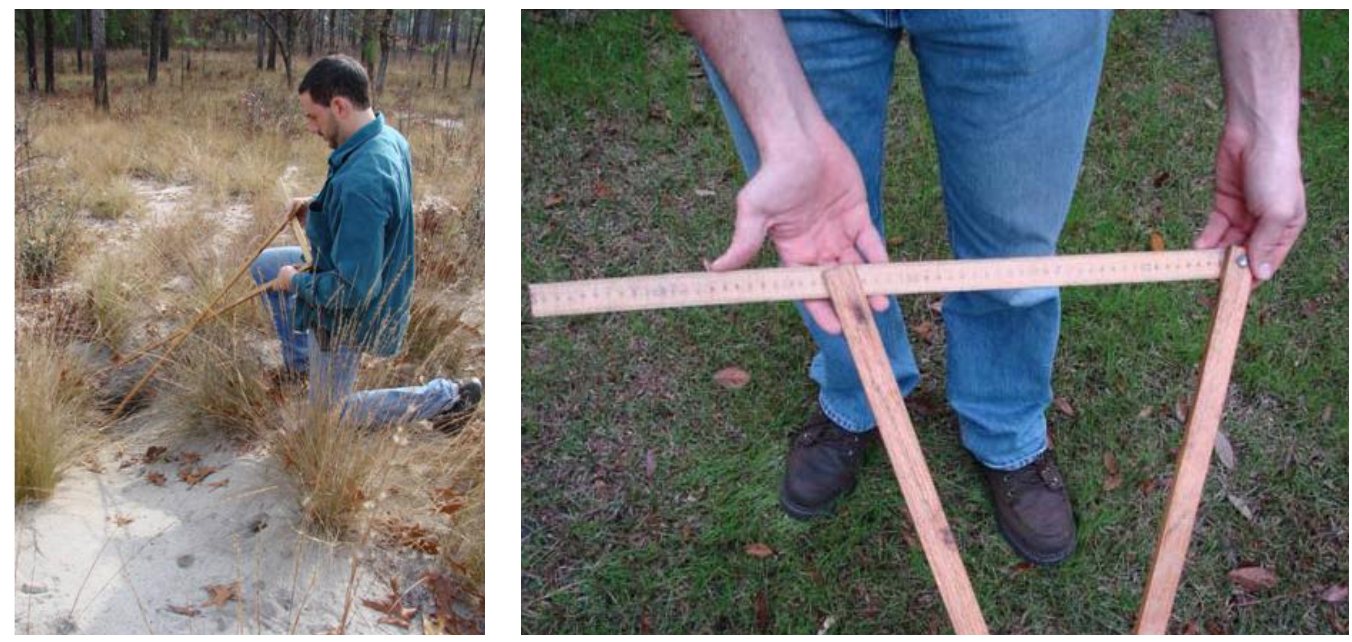

Figure 6. Use of calipers to measure the width of a gopher tortoise burrow. Measurements should be taken in metric units $(\mathrm{mm}$ or $\mathrm{cm}$ ) at $50 \mathrm{~cm}$ inside the burrow entrance.

Measuring both burrow width and height can help discriminate between tortoise and non-tortoise burrows based on width-to-height ratios (T. Tuberville, pers. comm.), although it is best to use well-trained observers. Observers should record observations of any burrow associates (commensal species) as well as tortoises when scoping burrows.

\section{Full Survey (high tech)}

The survey is created and conducted in the same format as in the basic survey above. However, rather than transferring the waypoints into a GPS 
unit, the XY coordinates are imported into ArcGIS (Tools - Add XY command) and are turned into a shapefile. The end points of the transects are then turned into lines (transects) using the points to lines command in Hawth's analysis tools (http://www.spatialecology.com/htools/). Digital photos of the survey site can be obtained through a GIS digital map clearinghouse. Load the digital photo(s), and the shapefiles of the points and transects onto the field computer, and then open ArcPAD and create a project composed of the shapefiles and photos. Create a point shapefile to collect, at a minimum, tortoise burrow data and transect start and end points. The field survey team can then navigate to the starting point of each transect with the GPS and navigate along the transect using the aerial photos visible on the screen. GPS points are taken for all burrows observed during surveys and the perpendicular distances from the centerline are calculated back in the office using ArcGIS. For this system to work it is critical that the equipment have a real time (WAAS signal) unit with sub meter accuracy. Real time WAAS signal facilitates navigation and allows collection of highly accurate location data.

In the field, one person should search the centerline and the area close to the centerline for burrows. Two additional observers should search on either side of the centerline weaving back and forth to detect burrows from the centerline outward. Once a burrow is encountered along the transect, a GPS location is taken at the burrow mouth and the associated attribute information is recorded [e.g., burrow depth, burrow width, occupancy, etc. (See Appendix A)]. This process is repeated at each tortoise burrow or tortoise observed in the field. It is also a good practice to collect a GPS point at the beginning and end of the surveyed transect to determine the actual length of transect surveyed (http://www.xtoolspro.com/ xtools can be used to calculate the length of transect actually surveyed in the field.) Using a GPS unit with less accuracy will introduce error and undermine the assumption that perpendicular distances are measure accurately. The burrow location shapefile will be transferred back to the desktop ArcGIS and a Near command performed to obtain the perpendicular distance from the transect line to the burrow. This information is then transferred over into Program Distance for analysis.

\section{Population monitoring}

If the survey protocols are consistently applied, periodic re-sampling at permanent transects can be used to monitor tortoise densities over time. This is particularly important when the objective is to monitor effects of management or restoration activities on tortoise populations. To detect 
trends, each sampling effort should yield a coefficient of variation that is 20 percent, or ideally between 10-15 percent. To detect population trends over a 20-year period, it is recommended that monitoring programs include re-sampling at 3- to 5-year intervals. Less frequent re-sampling will also allow for detection of trends, but only over a longer time scale. A more intensive and more frequent sampling regime may be required for critically endangered populations or populations perceived as declining, to detect and react to trends quickly. More intensive and frequent sampling may also be important to determine if populations are responding to newly implemented management practices.

Table 1. Metadata associated with standardized tortoise surveys.

\begin{tabular}{|c|c|}
\hline Transect \# & Unique ID of transect \\
\hline Linear distance along transect $(\mathrm{m})$ & $\begin{array}{l}\text { Distance along center-line to burrows-helps to relocate bur- } \\
\text { rows to be scoped. Not needed if GPS used. }\end{array}$ \\
\hline $\begin{array}{l}\text { Perpendicular distance from } \\
\text { centerline }(\mathrm{m})\end{array}$ & $\begin{array}{l}\text { Measure this consistently from burrow entrance to transect } \\
\text { center line. Not needed if GPS used. Use Near command to } \\
\text { calculate in ArcGIS. }\end{array}$ \\
\hline Burrow diameter $(\mathrm{cm})$ & $\begin{array}{l}\text { Measure the burrow width of burrow } 50 \mathrm{~cm} \text { inside the open- } \\
\text { ing, this measurement is a surrogate for tortoise length to } \\
\text { describe the demography of the population. Only data from } \\
\text { occupied burrows will be used in analyses. }\end{array}$ \\
\hline Burrow scoped (Yes or No) & Was an attempt made to scope this particular burrow? \\
\hline $\begin{array}{l}\text { Burrow occupancy (tortoise } \\
\text { observed, no tortoise, } \\
\text { undetermined) }\end{array}$ & $\begin{array}{l}\text { Confirm the results of burrow scoping. Record occupancy as } \\
\text { "no tortoise" only if the end of the burrow is clearly observed. } \\
\text { Record occupancy as undetermined unless the end of the } \\
\text { burrow is clearly identified or a tortoise is observed. Be } \\
\text { aware that tortoises can occupy flooded burrows, which can- } \\
\text { not be examined with a burrow scope. }\end{array}$ \\
\hline Burrow length (m) & $\begin{array}{l}\text { Can be measured using cable from burrow scope (measured } \\
\text { in half-meter intervals). }\end{array}$ \\
\hline Burrow activity status & $\begin{array}{l}\text { Active burrows have clear opening with tortoise tracks or } \\
\text { slide marks; Inactive burrows have clear opening to burrow, } \\
\text { but no tracks or slide marks; old burrows have debris in the } \\
\text { opening and may be partially or completely collapsed. }\end{array}$ \\
\hline Burrow content & List burrow associates observed, if any. \\
\hline Comments & List any additional comments or notes. \\
\hline
\end{tabular}




\section{Conclusion}

This handbook presents standardized methods for gopher tortoise surveys that will allow users to estimate population size and density and to monitor trends in tortoise populations over time. Chapter 2 outlines key steps for developing a tortoise population monitoring effort, including how to define suitable habitat (i.e., sampling frame) and criteria for choosing a sampling approach. Chapter 3 provides standardized methods for estimating tortoise densities and for long-term monitoring of the status and trends of populations using Line Transect Distance Sampling. Appendix A to this report provides two examples of potential applications of these methods. 


\section{References}

Alford, R. A. 1980. Population structure of Gopherus polyphemus in northern Florida. J ournal of Herpetology 14:177-182.

Anderson, D. R., K. P. Burnham, B. C. Lubow, L. Thomas, P. S. Corn, P. A. Medica, and R. W. Marlow. 2001. Field testing line transect estimation of desert tortoise abundance. J ournal of Wildlife Management 65:583-597.

Ashton, R. E., Jr., and P. S. Ashton. In Press. The Natural History and Management of the Gopher Tortoise (Gopherus polyphemus Daudin). Malabar, FL: Krieger Press.

Auffenberg, W., and R. Franz. 1982. The status and distribution of the gopher tortoise (Gopherus polyphemus), pp 95-126 in North American tortoises: Conservation and ecology. R. B. Bury (ed.). Wildlife Research Report 12. Washington, DC: U.S. Department of the Interior Fish and Wildlife Service.

Averill-Murray, R. C., and A. Averill-Murray. 2005. Regional-scale estimation of density and habitat use of the desert tortoise (Gopherus agassizii) in Arizona. J ournal of Herpetology 39:65-72.

Bausell, R. B., and Y.-F. Li. 2002. Power analysis for experimental research. New York: Cambridge University Press.

Breininger, D. R., P. A. Schmalzer, and C. R. Hinkle. 1991. Estimating occupancy of gopher tortoise (Gopherus polyphemus) burrows in coastal scrub and slash pine flatwoods. J ournal of Herpetology 25:317-321.

Buckland, S. T., D. R. Anderson, K. P. Burnham, J. L. Laake, D. L. Borchers, and L. Thomas. 2001. Introduction to distance sampling: Estimating abundance of biological populations. Oxford University Press, Great Britain.

Buckland, S. T., D. R. Anderson, K. P. Burnham, J. L. Laake, D. L. Borchers, and L. Thomas. 2004. Advanced distance sampling: Estimating abundance of biological populations. Oxford, England: Oxford University Press.

Burke, R. 1989. Burrow-to-tortoise conversion factors: Comparison of three gopher tortoise survey techniques. Herpetological Review 20:92-94.

Carthy, R. R., M. K. Oli, J. B. Wooding, J. E. Berish, and W. D. Meyer. 2005. Analysis of gopher tortoise population estimation techniques. ERDC/CERL TR-05-27. Champaign, IL: Engineer Research and Development Center, Construction Engineering Research Laboratory (ERDC-CERL)

Cox, J., D. Inkley, and R. Kautz. 1987. Ecology and habitat protection needs of gopher tortoise (Gopherus polyphemus) populations found on lands slated for largescale development in Florida. Unpublished report. Office of Environmental Service, Florida Game and Freshwater Fish Commission, Tallahassee, FL. 
Krzysik, A. J. 2002. A landscape sampling protocol for estimating distribution and density patterns of desert tortoises at multiple spatial scales. Chelonian Conservation and Biology 4:366-379.

Martin, P. L., and J. L. Layne. 1987. Relationship of gopher tortoise body size to burrow size in a southcentral Florida population. Florida Scientist 50:264-267.

McCoy, E. D., and Mushinsky, H. R. 1992. Studying a species in decline: gopher tortoises and the dilemma of "correction factors." Herpetologica 48:402-407.

Nichols, J. D., J. E. Hines, J. R. Sauer, F. W. Fallon, J. E. Fallon, and P. J. Heglund. 2000. A double-observer approach for estimating detection probability and abundance from point counts. Auk 117:393-408.

Pollock, K. H., H. Marsh, L. L. Bailey, G. L. Farnsworth, T. R. Simons, and M. W. Alldredge. 2004. Separating components of detection probability in abundance estimation: An overview with diverse examples. Pages $43-58$ in Sampling Rare or Elusive Species, W. L. Thompson, ed. Washington, DC: Island Press.

Smith, R. B., T. D. Tuberville, A. L. Chambers, K. M. Herpich, and J. E. Berish. 2004. Gopher tortoise burrow surveys: External characteristics, burrow cameras, and truth. Applied Herpetology 2:161-170.

Swann, D. E., R. C. Averill-Murray, and C. R. Shwalbe. 2002. Distance sampling for Sonoran desert tortoises. J ournal of Wildlife Management 66:969-975.

Thomas, L., J. L. Laake, S. Strindberg, F. F. C. Marques, S. T. Buckland, D. L. Borchers, D. R. Anderson, K. P. Burnham, S. L. Hedley, J. H. Pollard, and J. R. B. Bishop. 2003. Distance 4.1 Research Unit for Wildlife Population Assessment. University of St. Andrews, UK.

Witz, B. W., and D. S. Wilson. 1991. Distribution of Gopherus polyphemus and its vertebrate symbionts in three burrow categories. American Midland Naturalist 126:152-158. 


\section{Appendix A: Survey Examples using Line Transect Distance Sampling}

The examples that follow should be accompanied by the help provided in the Distance software as well as Buckland et al. 2001 and 2004. Refer to the following web site for details of distance sampling and software: http://www.ruwpa.st-and.ac.uk/distance/

\section{Example 1: Use of conventional distance sampling techniques on small to large tracts of land}

This example demonstrates use of conventional distance sampling techniques and can be applied on small (100 ha) to large (100,000 ha) tracts of land with extensive potential tortoise habitat, where observations are likely to approach 60-80 tortoises. This example presents a survey design from an 11,500 ha property with about 7700 ha of suitable tortoise habitat. The steps in designing the survey are outlined below.

\section{Define the goals and objectives of the survey}

Goals: To evaluate and track demographic changes in a large gopher tortoise population. Specifically, the goal is to develop and implement a sitewide monitoring protocol for gopher tortoises and to describe the population structure, estimate density and abundance, and detect trends. These data will be used to evaluate management strategies (including habitat restoration) for the species.

Objectives: To be able to detect a significant trend in gopher tortoise population size across the site over a 10-year time frame, and to derive population estimates with a $\mathrm{CV}<20$ percent.

\section{Define the population to be sampled}

The term population is used to designate the area from which the sample is taken; statistical inference is limited to the sampled population. In this case, the population is the tortoises found within 7700 ha of suitable habitat within the property boundary. 


\section{Choose a sampling method}

Line transect distance sampling (Buckland et al. 2001).

\section{Data to be collected}

Table A1 lists the data collected for this example. It is a best data management practice to design a database and to create a datasheet with associated metadata prior to initiating surveys. Ideally, the database should have pulldown menus and overwrite protections to aid in data entry and quality control. Data for long-term monitoring studies will be collected infrequently, and consistency in data collection is critical; therefore, it is important to clearly define metadata associated with a project, and it is critical for the database to have sufficient storage capacity for potentially large data sets. The best technology currently available for management of spatial data is a Global Positioning System with real time correction and a field computer and ArcGIS.

\section{Define the sampling frame}

The sampling frame (the area to be sampled, see Figure A1) can be based on property boundaries, management boundaries, habitat, soils, or other data. If there are potentially large differences in tortoise population densities across the area to be sampled, sampling should be stratified by logical variables such as management regime, habitat type, soil type, etc. For this example, the property boundary is defined as the sampling frame, and then identified and removed unsuitable habitat (active wildlife food plots, floodplain, and wetlands) from within the frame. It uses an "equal probability" design, which means that the sample was drawn from the entire area of suitable habitat within the sampling frame.

\section{Design and conduct a pilot survey}

The purpose of the pilot survey is to determine the encounter rate for tortoises on the site. The encounter rate is the number of animals observed along a particular length of transect, which is used to calculate the sampling intensity needed to obtain a density estimate with a specified CV (in this example $<20$ percent). For the pilot survey, it was proposed to survey approximately $4000 \mathrm{~m}$ of transects. Thirteen transects, totaling $>4000 \mathrm{~m}$ in length, were randomly placed across the site (Figure A2). 


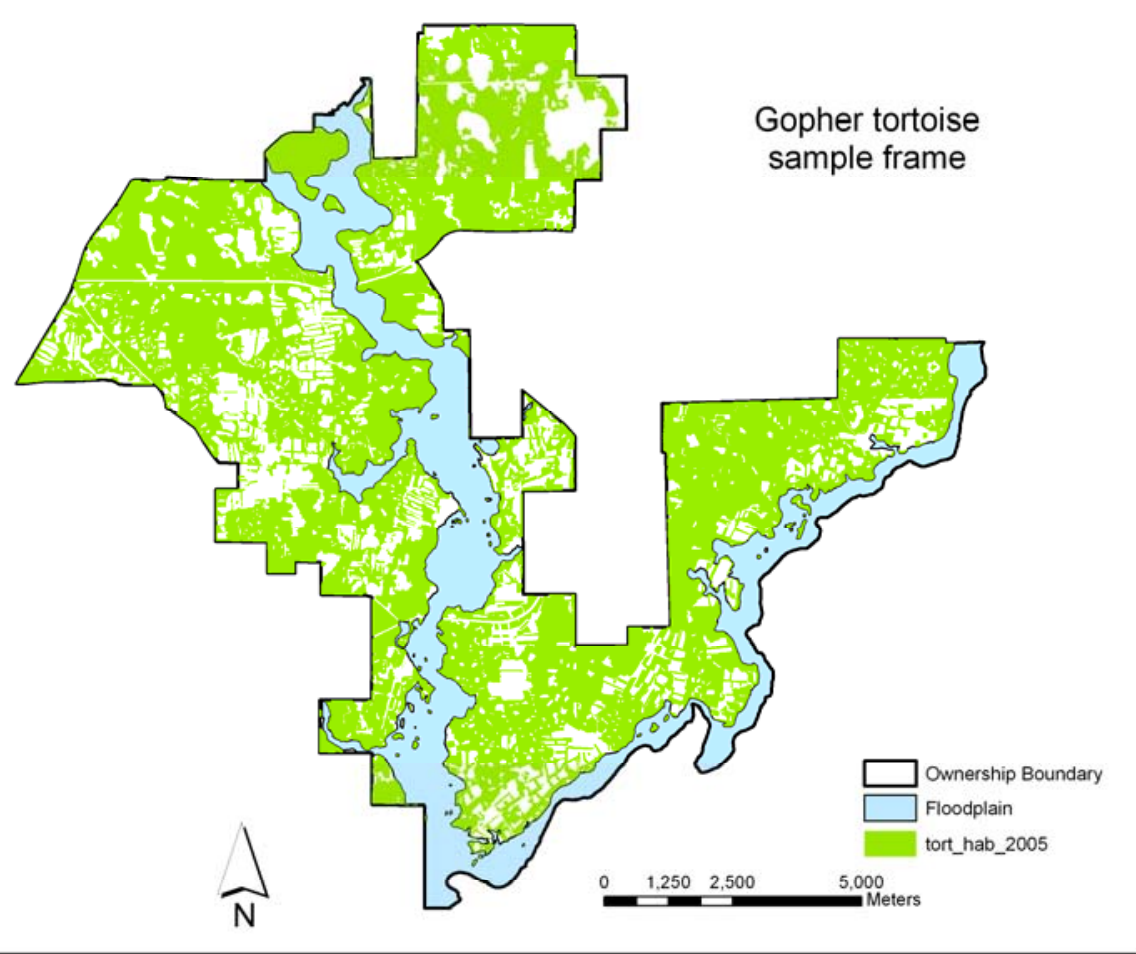

Figure A1. Sampling frame (in green), which includes the entire site, excluding unsuitable tortoise habitat: flood plain, wetlands, agricultural areas and wildlife food plots.

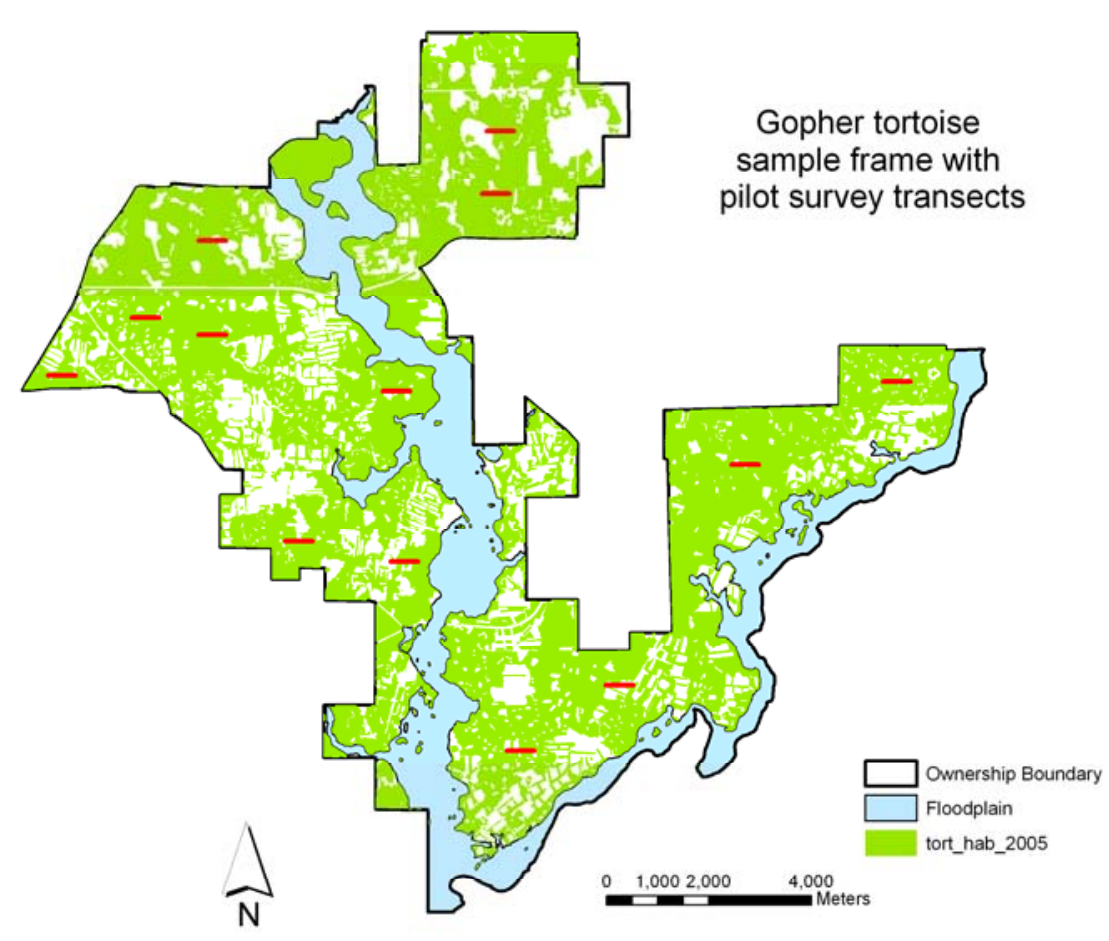

Figure A2. Distribution of pilot survey transects. 
The pilot survey used the LTDS method to survey the transects (depicted above) and to locate tortoises. Perpendicular distance measurements are not needed for calculating the tortoise encounter rate.

The formula for calculating the encounter rate is:

$L_{0} / n_{\circ}$

where:

$L_{o}=$ the total length of the transects

$n_{o}=$ the number of tortoises encountered.

In the pilot survey, 23 tortoises $\left(\mathrm{n}_{0}\right)$ were encountered on $4628 \mathrm{~m}\left(\mathrm{~L}_{\mathrm{o}}\right)$ of transect. Therefore, the encounter rate was:

$4628 / 23=201$

To determine the total length of transects (L) needed to achieve an estimate with a CV of 15 percent, use the following formula:

$$
L=\left(b / c v(D)^{2}\right) \times\left(L_{0} / n_{0}\right)
$$

where:

$$
\begin{aligned}
\mathrm{D}= & \text { density } \\
\mathrm{cv}(\mathrm{D})= & \text { the desired cv for the density estimate } \\
\mathrm{b}= & \text { the dispersion parameter (or the variance inflation factor), which } \\
& \text { for planning purposes can be treated as a constant value of } 3 \text { (See } \\
& \text { pg } 241 \text { in Buckland et al. [2001] for discussion). }
\end{aligned}
$$

For this example:

$$
L=\left(3 /(0.15)^{2}\right) \times 4628 / 23=26,800 \mathrm{~m}(\text { or } \sim 27 \mathrm{~km})
$$

The transect length (L) should be rounded up, in this case to $27 \mathrm{~km}$. It would be necessary to survey at least $27 \mathrm{~km}$ of transects across the site to achieve a CV of 15 percent. (Note: the selection of a CV of 15 percent was arbitrary, but for most studies, it is desirable to obtain a CV between 10 and 20 percent).

Alternatively, the encounter rate can be used to calculate the expected CV if, for example, only 20,000 $\mathrm{m}$ of transect could be sampled due to budgetary constraints. In this case, with $20,000 \mathrm{~m}$ of transect, the $\mathrm{CV}$ would be 17 percent, which is an acceptable value for most ecological studies:

$$
\operatorname{cv}(\mathrm{D})=\left(\mathrm{b} /(\mathrm{L}(\mathrm{no} / \mathrm{Lo}))^{1 / 2}=\left(3 /(20,000(23 / 4628))^{1 / 2}=0.1737 * 100=17.37 \%\right.\right.
$$




\section{Selection of the sample}

The encounter rate from the pilot study described above was used to develop a survey design in Program Distance. The sampling frame has already been imported as a GIS shape file into Distance for the pilot survey. Follow the instructions below to design the full survey. Consult the manual to import a shape file of the sampling frame and grid construction. Note: Before using Program Distance, it is strongly recommended that users review the manual (including the example data sets provided) to understand how data is structured and stored. The Users Guide and Help menu in Program Distance provides instruction on how data and projects are stored, and how to import GIS shape files into Distance.

\section{Survey design}

In Program Distance, under the design tab "Type of Design" header, select the type of sampler as "Line" and class as "systematic segmented grid sampling” (Figure A3).

Then click on the properties box to the right and make the following selections. On the Effort Allocation tab under edge sampling select "Plus" (Figure A4). By selecting Plus, you will add additional transect length (the half width of your transect) at the edge of the sample frame to prevent underestimating populations distributed along edges of the area to be sampled. If you do not select "plus" samplers, you risk biasing your survey. Under "Sampler Segment Handling" there are several options, depending on how fragmented the sample frame is. Typically, one should allow for split samplers, but discard samplers that are $<25$ percent of a standard transect length.

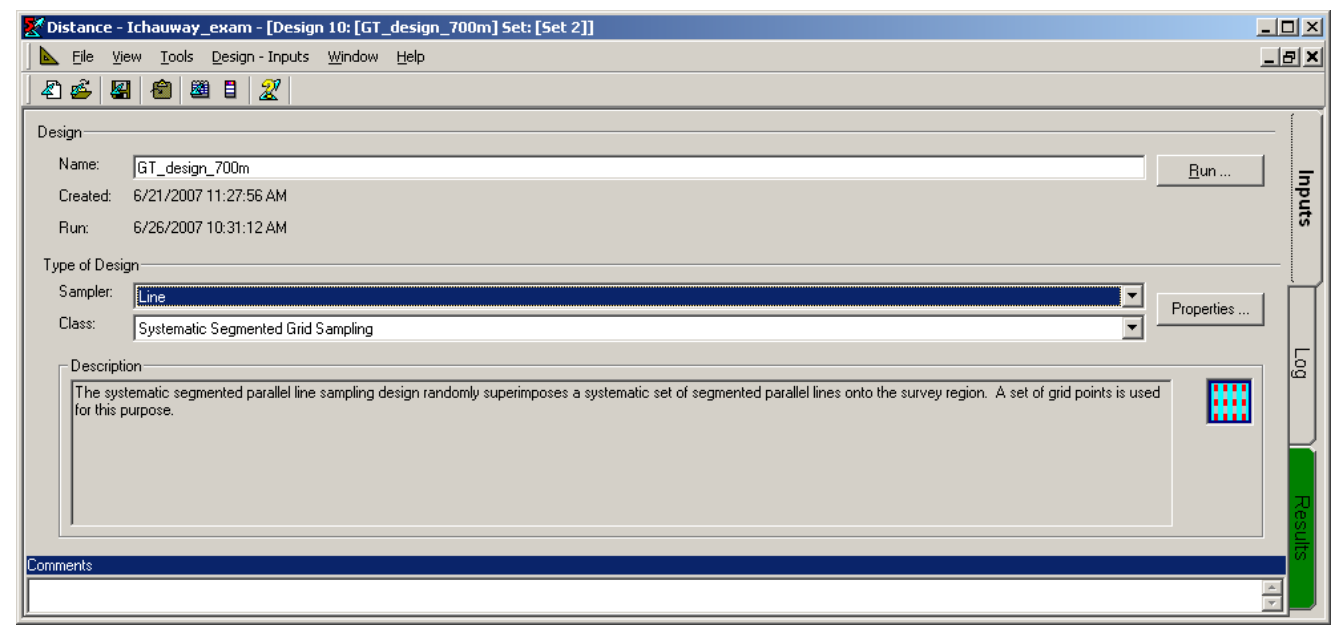

Figure A3. “Type of Design” menu. 


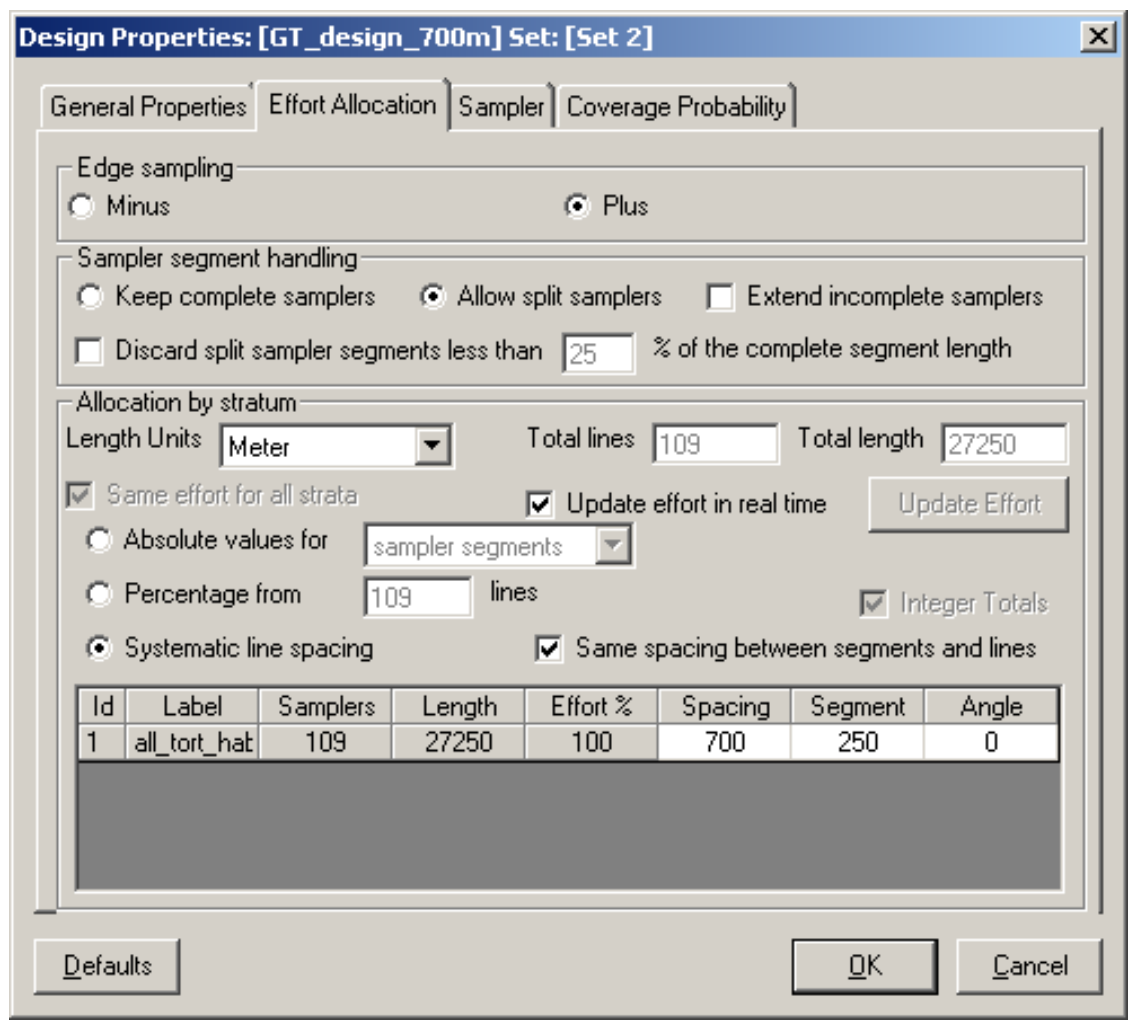

Figure A4. Effort Allocation tab.

Under "Allocation by stratum," unequal probability sample designs can be created for different strata. However, this example uses an Equal probability sample. Start by placing transects at $700 \mathrm{~m}$ intervals under the Spacing column. Under the Segment column, use a standard transect length of $250 \mathrm{~m} ; 250 \mathrm{~m}$ were selected as the standard transect length so that most transects fall within one burn unit, so the entire transect could be surveyed after a prescribed fire. However, transects can be of any length or even continuous across the site. In the Angle column, you may specify the orientation of the transects. Leaving the Angle at "o" provides an east-west transect orientation. Once values have been entered for the variables outlined above, the results will give the number of samplers and the length of transects surveyed. At this point, you can adjust transect spacing or length to achieve the total transect length estimated in the pilot survey.

Go to the Sampler tab (Figure A5) and enter half the width of the transects to be surveyed, e.g., $20 \mathrm{~m}$ (in other words a total sampling width of $\sim 40 \mathrm{~m}$ ). This does not mean you are restricting sampling in the field to a width of $20 \mathrm{~m}$ around the center-line, rather, this is just an estimate of the area that will be covered by the survey crew. This can also be thought of as the surveys expected strip width (ESW). On the final tab (Coverage Probability) make the selections shown in Figure A6. 


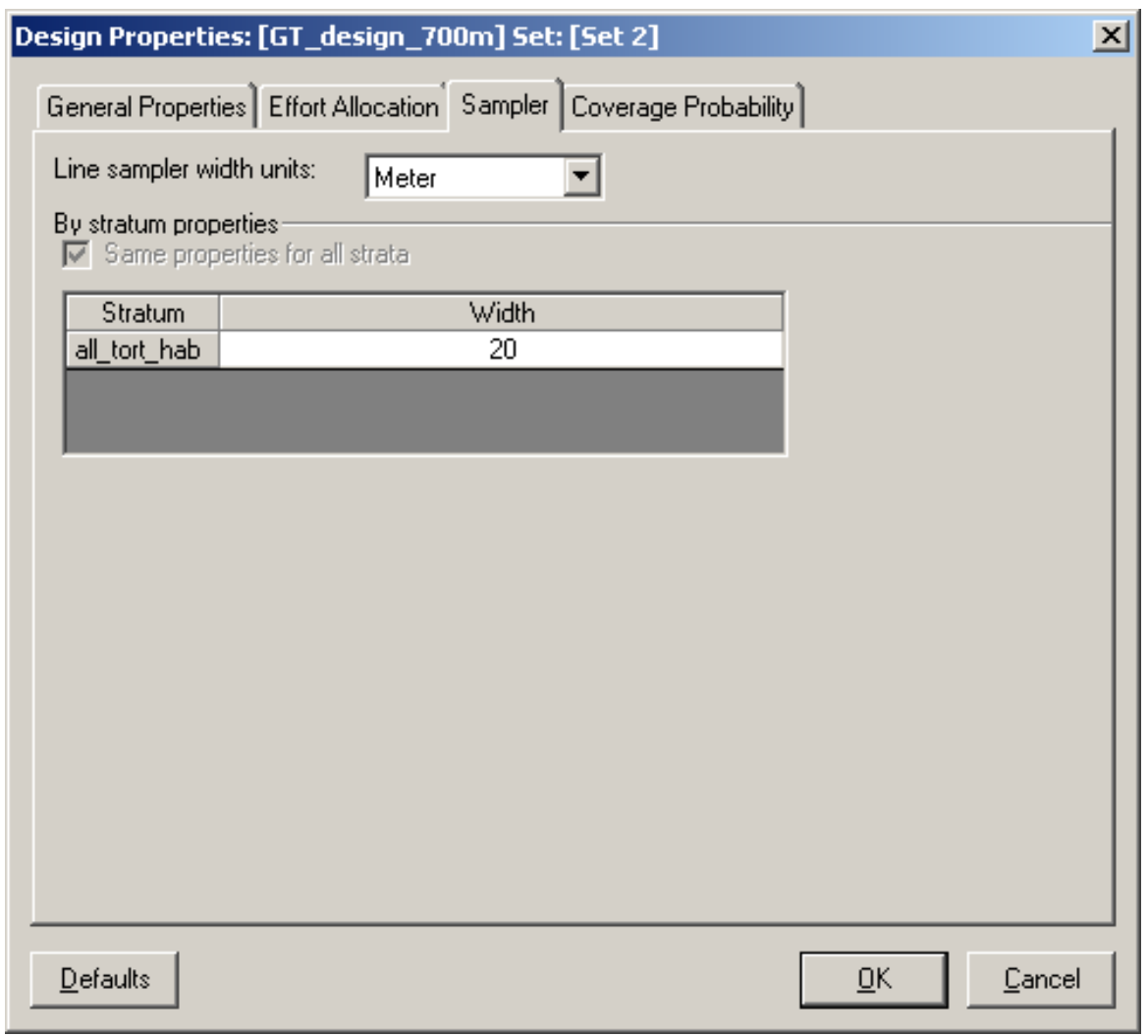

Figure A5. "Sampler" tab of the Design Properties menu.

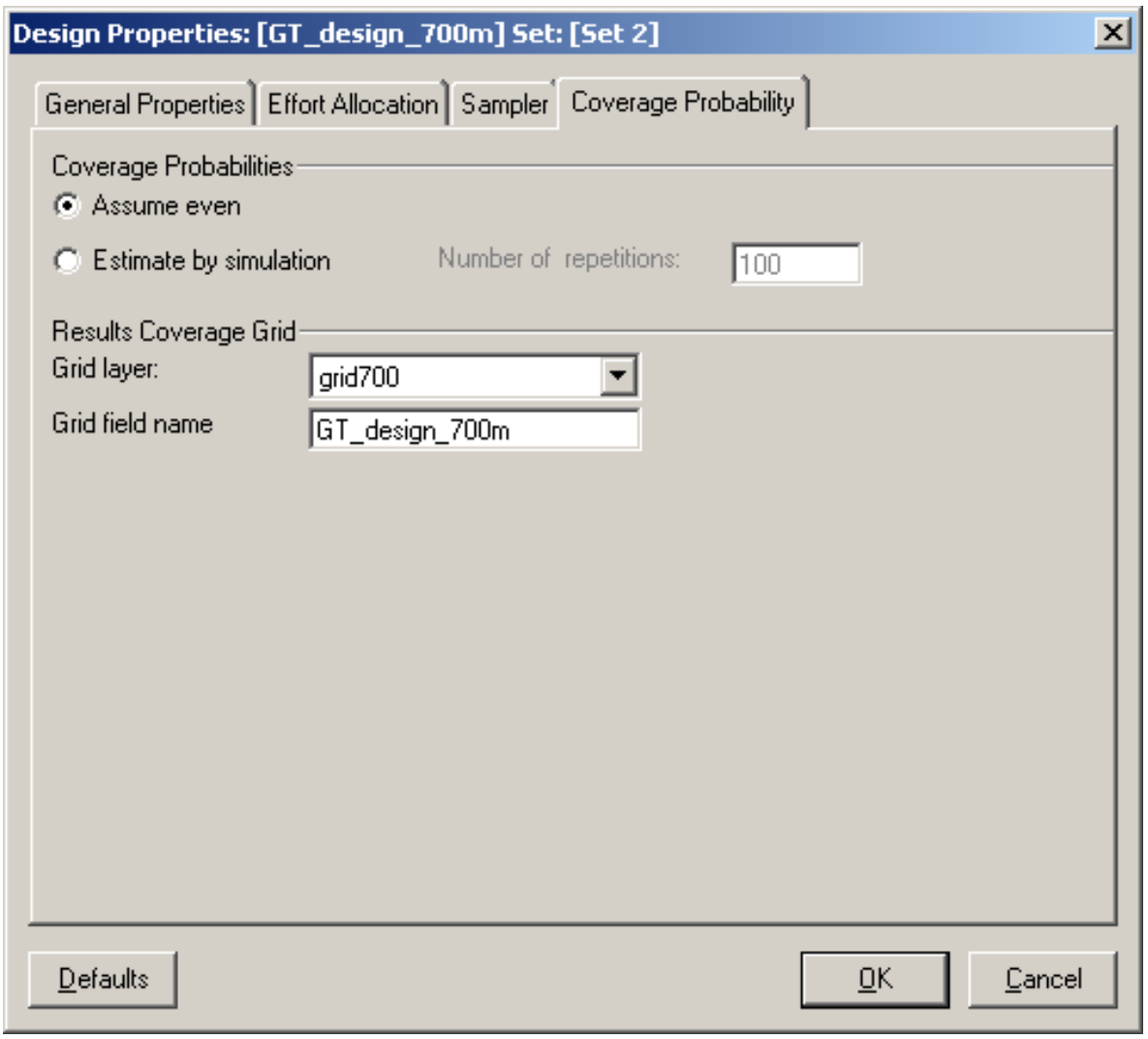

Figure A6. "Coverage Probability" tab of the Design Properties menu. 
Assume coverage probabilities are even, select a grid, and then click $\underline{\mathrm{OK}}$. Then run the program. Next click $\underline{\mathrm{OK}}$ and run the design. Then go to the Survey tab and take this design and create a survey. You should get the output shown in Figure A7.

The survey output (Figure A7) will summarize the amount of area to be surveyed, the proportion of the area to be surveyed, and other details. You will now take this design and go to the Survey tab.

Survey tab: Select the design created in the last step (Figure A8).

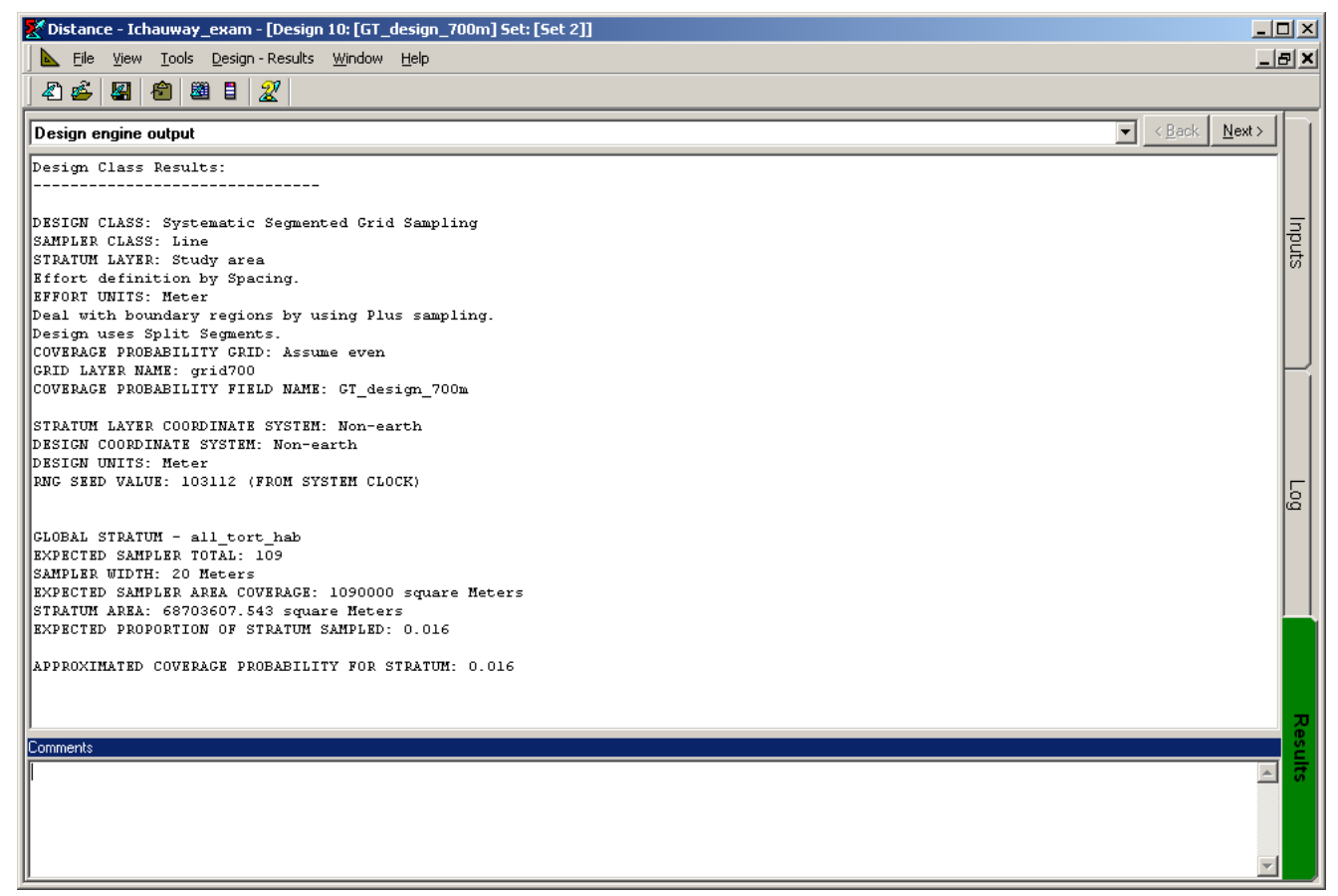

Figure A7. Survey output.

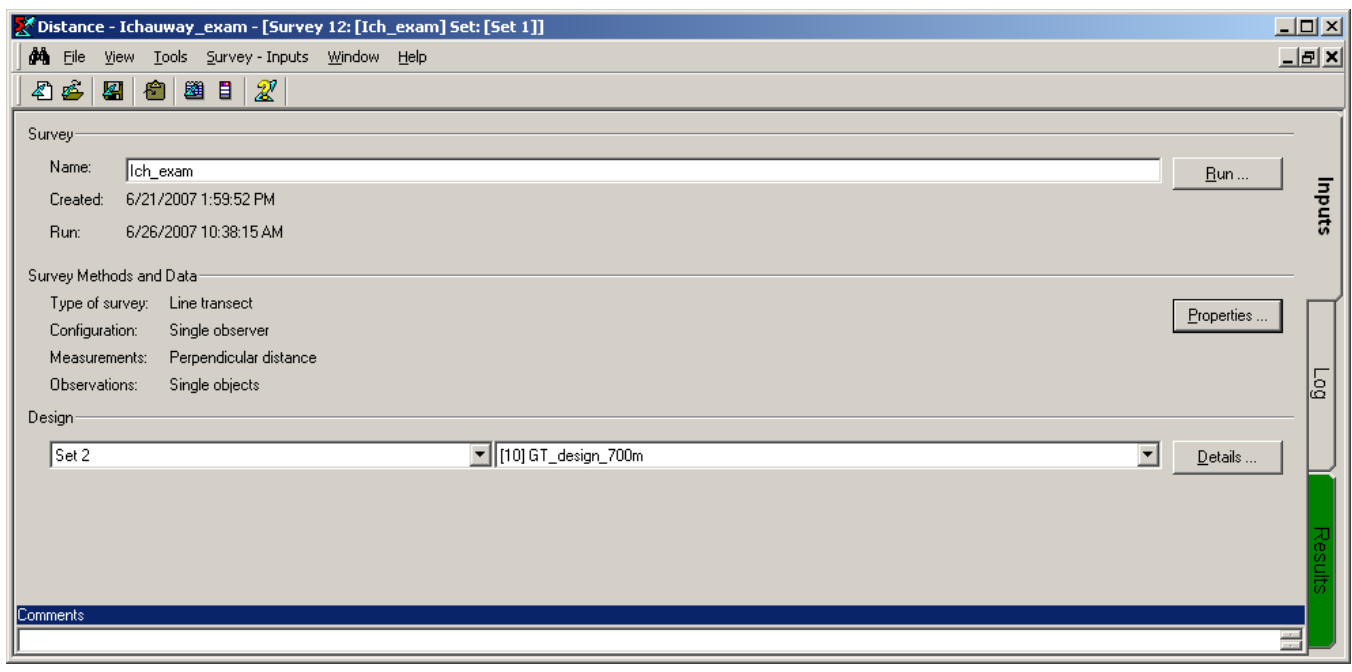

Figure A8. Survey tab. 
Click on properties button on the right and select the following options.

Under Survey methods and Data (Figure A9) chose all of the following options: "Line transect," "Single Observer," "Perpendicular distance," and "Single objects."

Select the Design in the pulldown boxes under "Design." Name the survey and click Run. After the job finishes, you will have three output pages: a summary page, a map with transects (Figure A10), and the location of the start and end points of the transects. You can take the XY coordinates of the transects, copy them into Notepad or Excel and convert them into an appropriate format for import into a GPS as waypoints and to display in a GIS.

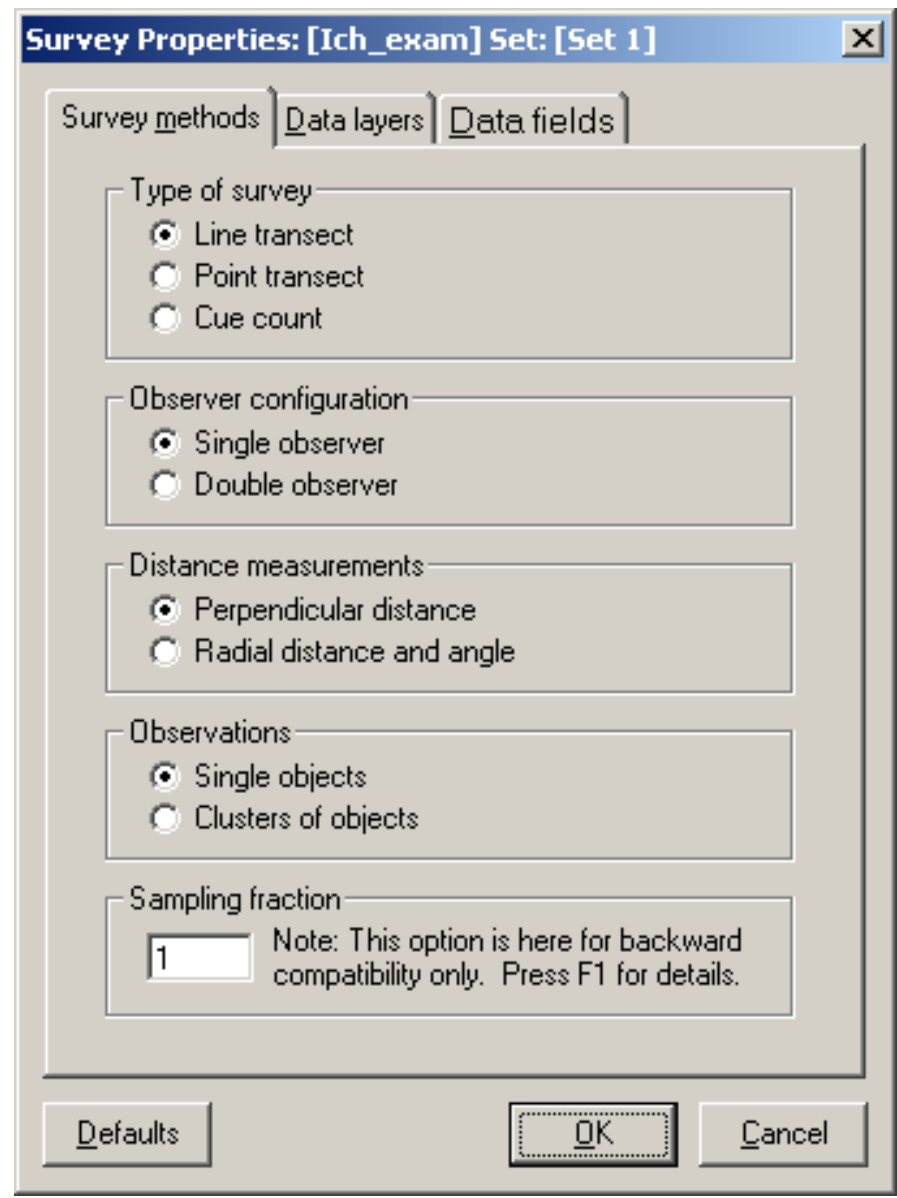

Figure A9. Survey methods tab. 


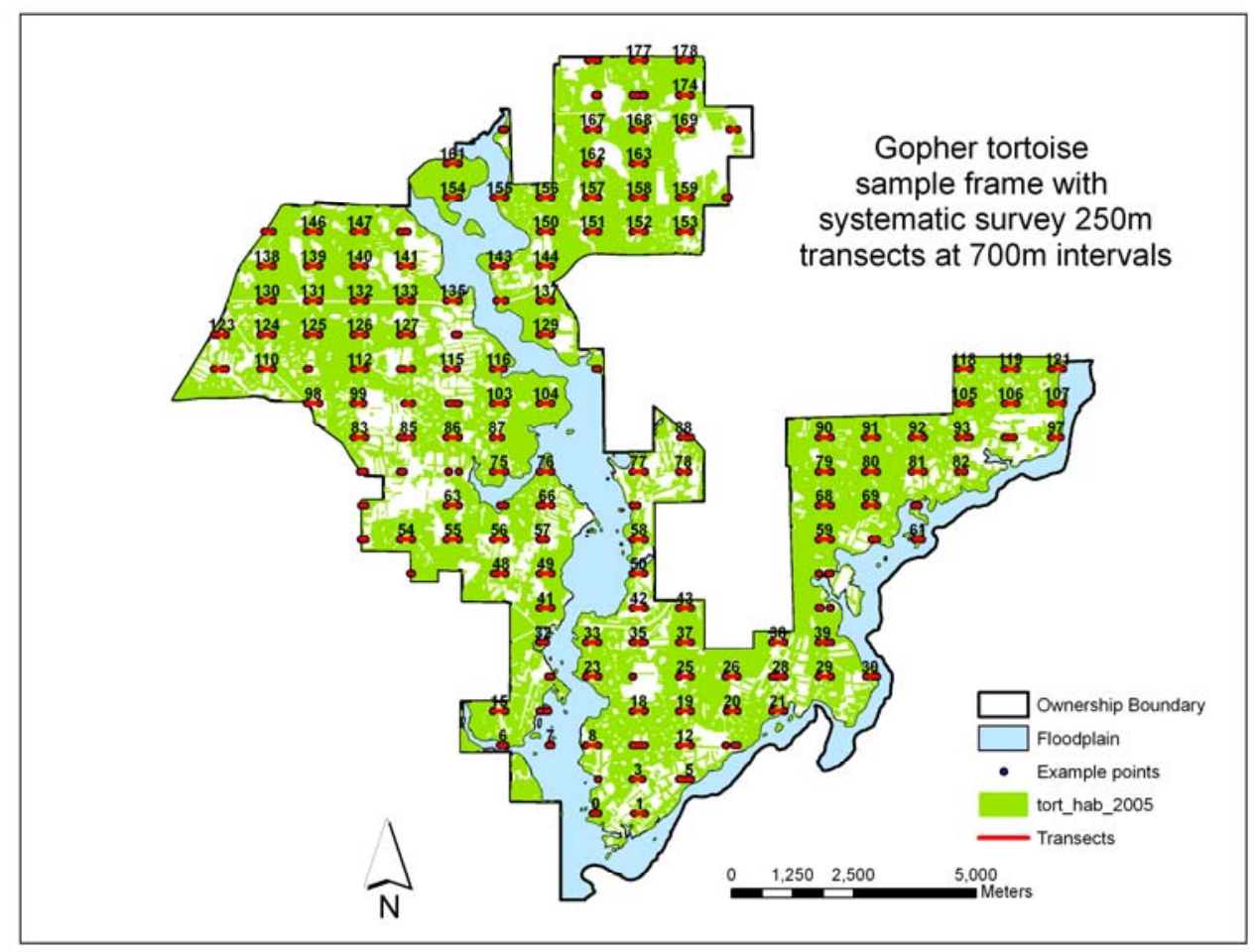

Figure A10. Equal probability sample with $250 \mathrm{~m}$ transects spaced at $700 \mathrm{~m}$ intervals. The eastern terminus of each transect is identified as the starting point.

\section{8) Summary and analysis of the data}

See example below (Step 9) for details on analysis of distance data.

\section{Example 2. Methods for sampling small populations}

This example demonstrates the use of conventional distance sampling for small tracts of habitat with relatively few tortoises. The example is based on actual surveys of a 48 ha property with an unknown number of gopher tortoises.

\section{Define the goal and objective of the survey}

Goal: Obtain density and abundance estimates for gopher tortoises on the site.

Objective: To obtain an estimate of gopher tortoise density and abundance with the lowest attainable CV. Note: With a small population, it is likely impossible to achieve the same rigor (e.g., $\mathrm{CV}<15$ percent) as with a large sample. Additional sampling may be conducted to achieve better esti- 
mates. For example, repeated surveys or re-sampling of the transects can be used to increase the precision of the estimate.

\section{Define the sample population}

Property boundary is outlined in blue and suitable tortoise habitat is designated in red (Figure A11). As before, sampling only suitable habitat is a necessity for accurate results.

\section{Method of measurement}

Do line transect distance sampling in the same format as the survey example above.

\section{Data to be collected}

See Table 1 (p 17).

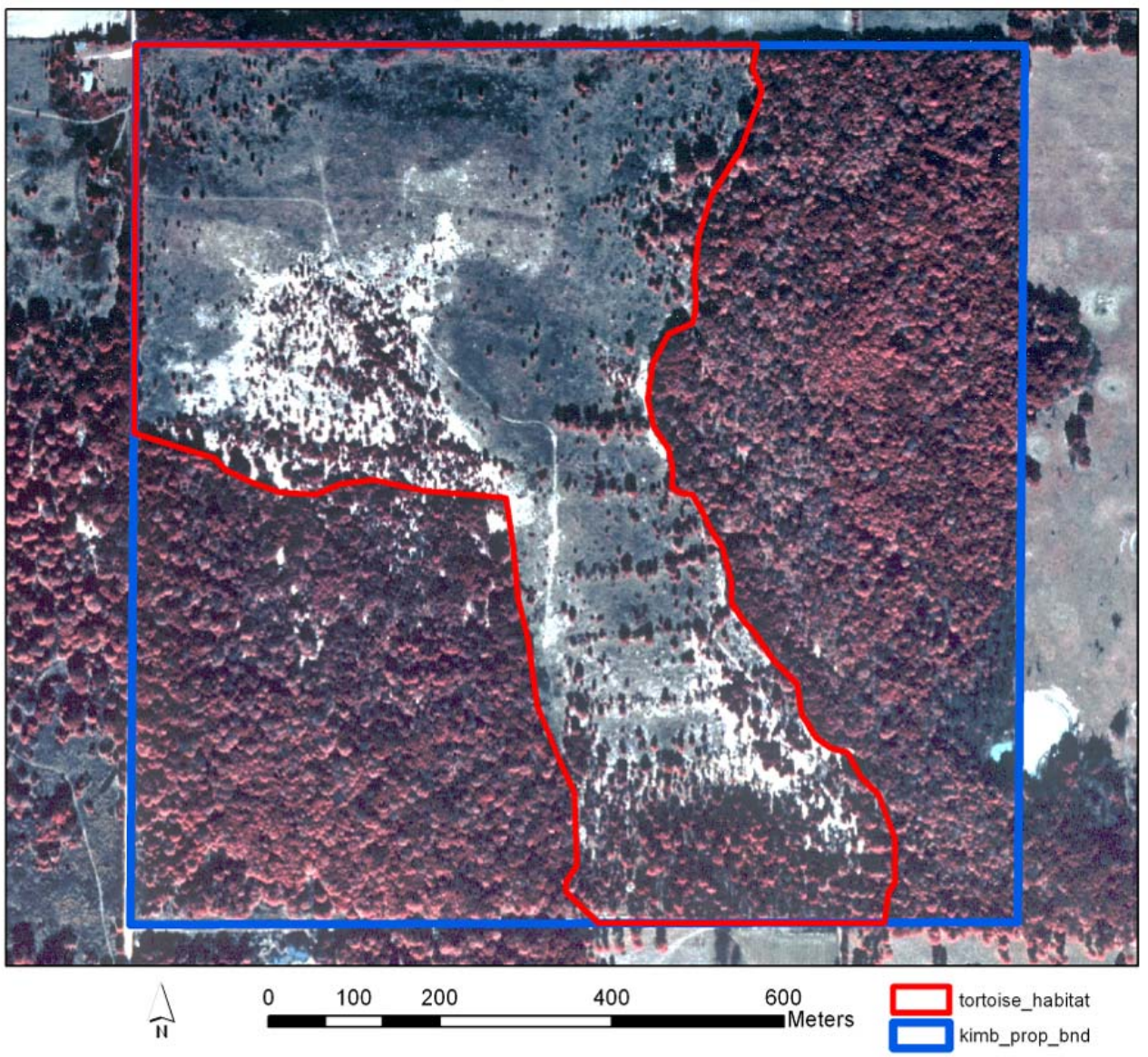

Figure A11. Sample population example. 


\section{Define the sampling frame}

The site has 48.7 ha of suitable gopher tortoise habitat and is comprised of abandoned Bahia grass (Paspalum notatum) pasture, a sand ridge with sand live oak scrub, and a patch of mature longleaf pine designated in the above figure as tortoise habitat. The remaining habitat is dominated by hardwood community over mesic flatwoods soils and is unsuitable habitat for tortoises.

\section{Design and conduct pilot survey}

Four transects that totaled $800 \mathrm{~m}$ in length (Figure A12) were surveyed to determine the tortoise encounter rate. Four tortoises were detected and the site has 48.7 ha of suitable habitat. The following calculation was done to estimate the length of transect needed to achieve a 15 percent CV:

$$
\begin{aligned}
& \mathrm{n}_{\circ}=4, \mathrm{~L}_{\circ}=800 \mathrm{~m}, \mathrm{~b}=3 \text {, and } \operatorname{cv}(\mathrm{D})=15 \% \\
& \mathrm{~L}=\left(\mathrm{b} / \mathrm{cv}(\mathrm{D})^{2}\right) \times\left(\mathrm{L}_{\circ} / \mathrm{n}_{\circ}\right) \\
& \mathrm{L}=\left(3 /(0.15)^{2}\right) 800 / 4=26,666 \mathrm{~m}
\end{aligned}
$$

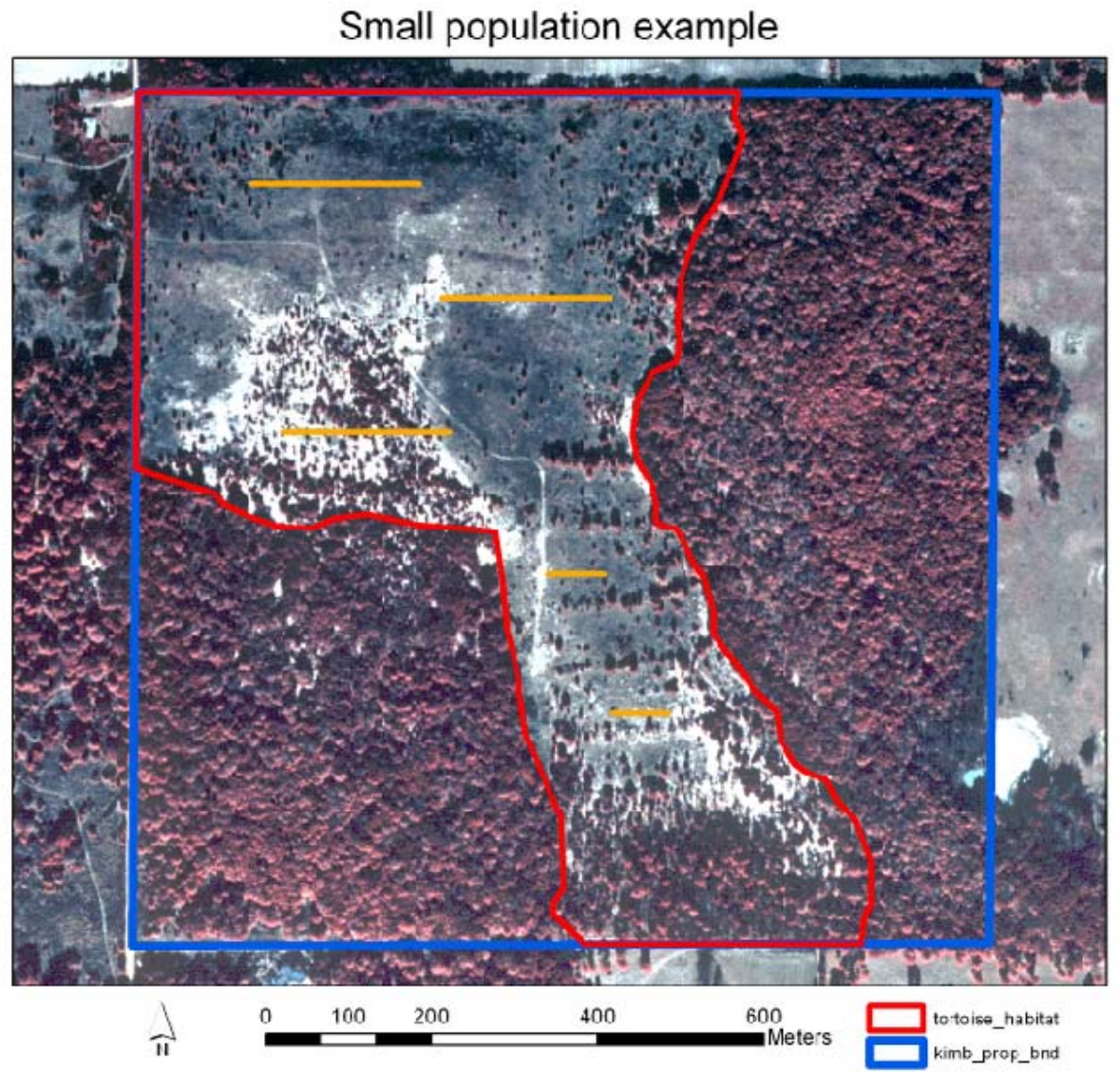

Figure A12. Available gopher tortoise habitat and pilot survey design. 
From this calculation, it was clear that achieving a 15 percent $\mathrm{CV}$ would not be feasible with a single survey since there were only $48,700 \mathrm{~m}^{2}$ of suitable habitat on the site. Therefore, the CV that could be obtained by sampling what was considered to be a reasonable survey effort $(10,800 \mathrm{~m}$ of transect) was back-calculated. Assuming a total width of the transect as $40 \mathrm{~m}$ for a total transect length of $10,800 \mathrm{~m}$, would cover $10800^{*} 40=$ $432,000 \mathrm{~m}^{2} / 10,000 \mathrm{~m}^{2} / \mathrm{ha}=43.2 \mathrm{ha}$, or 88 percent of the site. It took a field crew about $3 \mathrm{hrs}$ to survey $800 \mathrm{~m}$ of transect for the pilot survey; therefore, it was estimated that it would take $33.75 \mathrm{~h}$ (or about 4 days) to conduct the full survey using the basic protocol as compared to only 1 day using the high tech protocol.

To determine the CV obtained using a transect length $(\mathrm{L})=10,800 \mathrm{~m}$ :

$$
\operatorname{cv}(D)=(b /(L(n o / L o)))^{1 / 2}=\left(3 /(10,800(4 / 800))^{1 / 2}=0.235 * 100=23.5 \%\right.
$$

\section{Selection of the sample}

The shape file was imported into Distance and a design and survey were created (Figure A13). See the Distance Help for creating a new project, importing a shape file into distance, creating a grid, and viewing maps in distance.

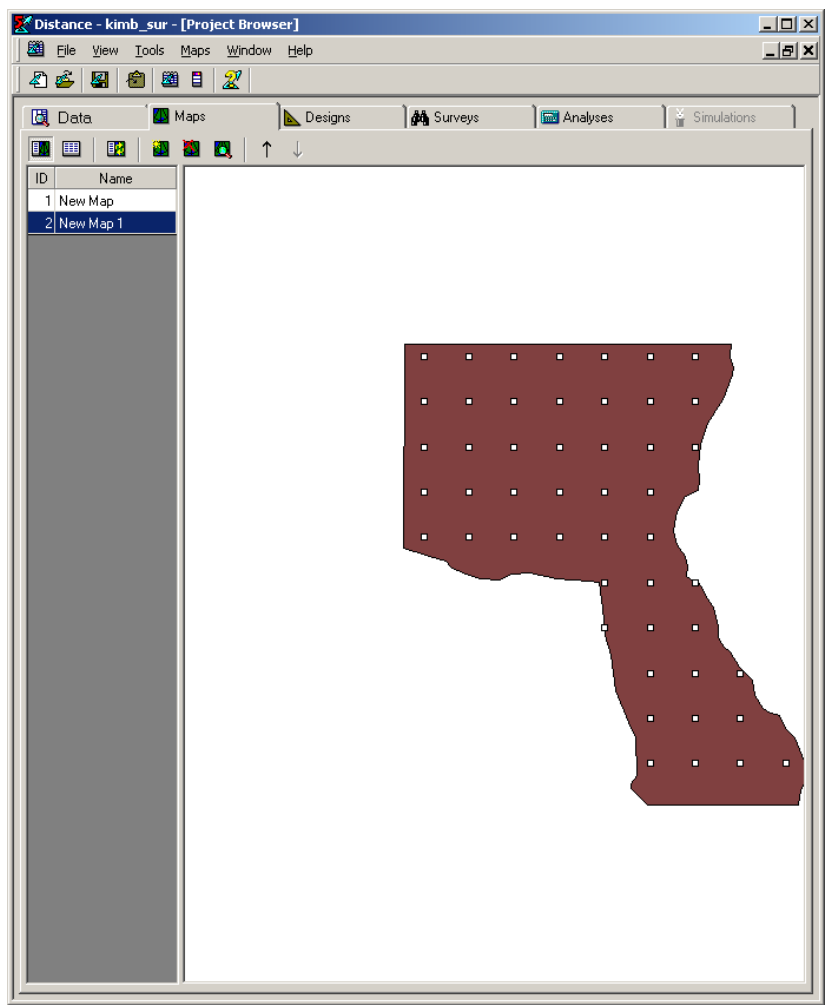

Figure A13. Shape file. 
Once the project is created and a shape file imported in Distance, move to the Design tab to generate a design. See above example for choices to be made in the design tab. Go to the Properties tab and set the defaults highlighted in Figure A14, then go to the Details and select the Survey type; in this instance, systematic parallel design was selected.

Running the design module yields the results above, which include $\sim 10,439 \mathrm{~m}$ of transect covering 80 percent of the site with 21 transects placed $50 \mathrm{~m}$ apart with a $20 \mathrm{~m}$ half width. There is a total transect width of $40 \mathrm{~m}$ leaving $\sim 10 \mathrm{~m}$ between transects to avoid double counting burrows. The survey design will provide coordinates for the start and end points of transects (Figure A15) that can be modified and ultimately imported into a GPS unit. Pilot study results (Figures A16 and A17) arrived at close to the $10,888 \mathrm{~m}$ of transect.
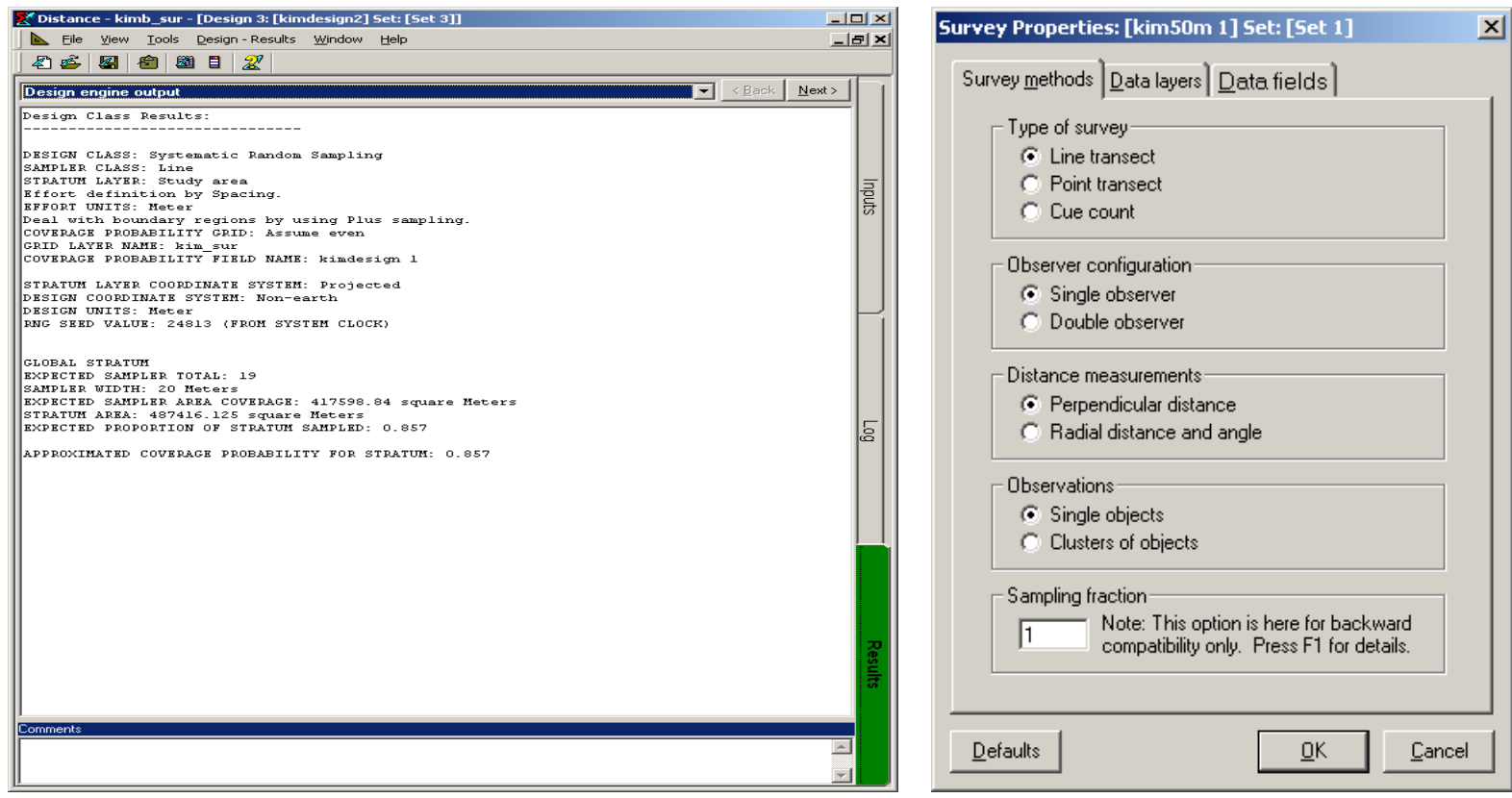

Figure A14. Survey method selection. 


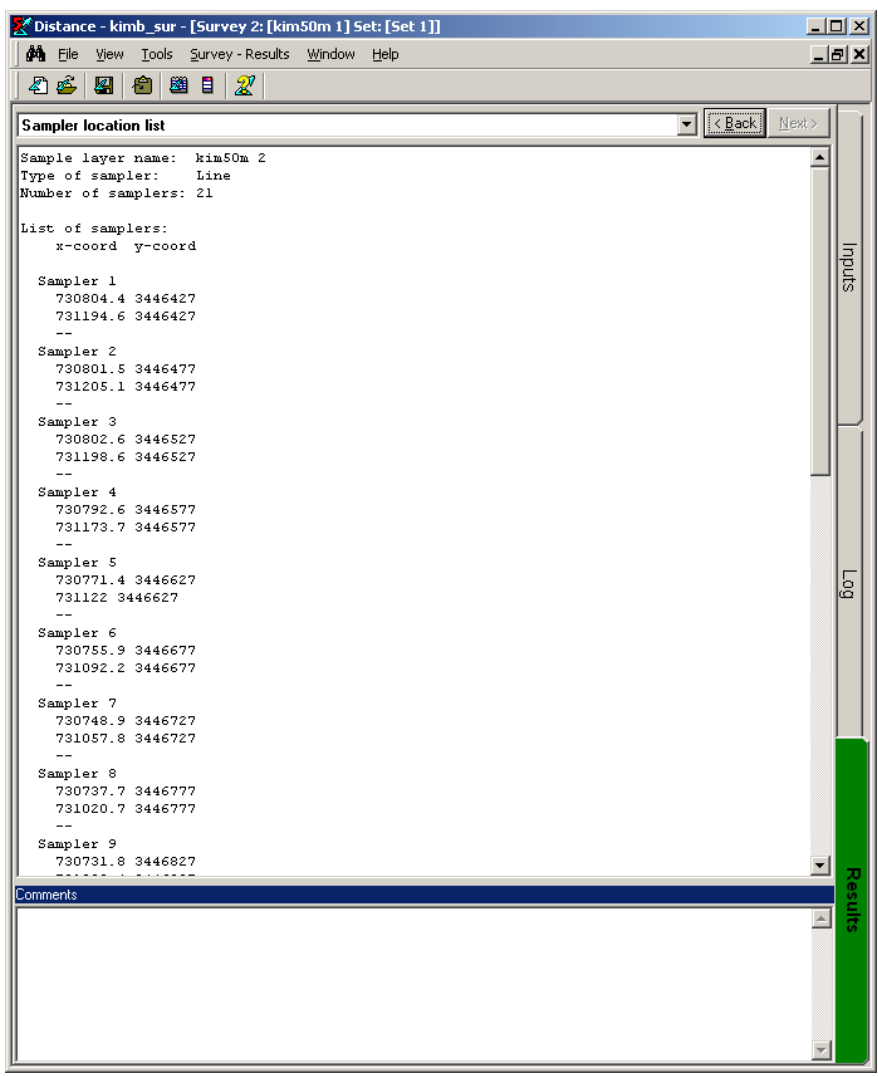

Figure A15. Start and end point coordinates of transect.

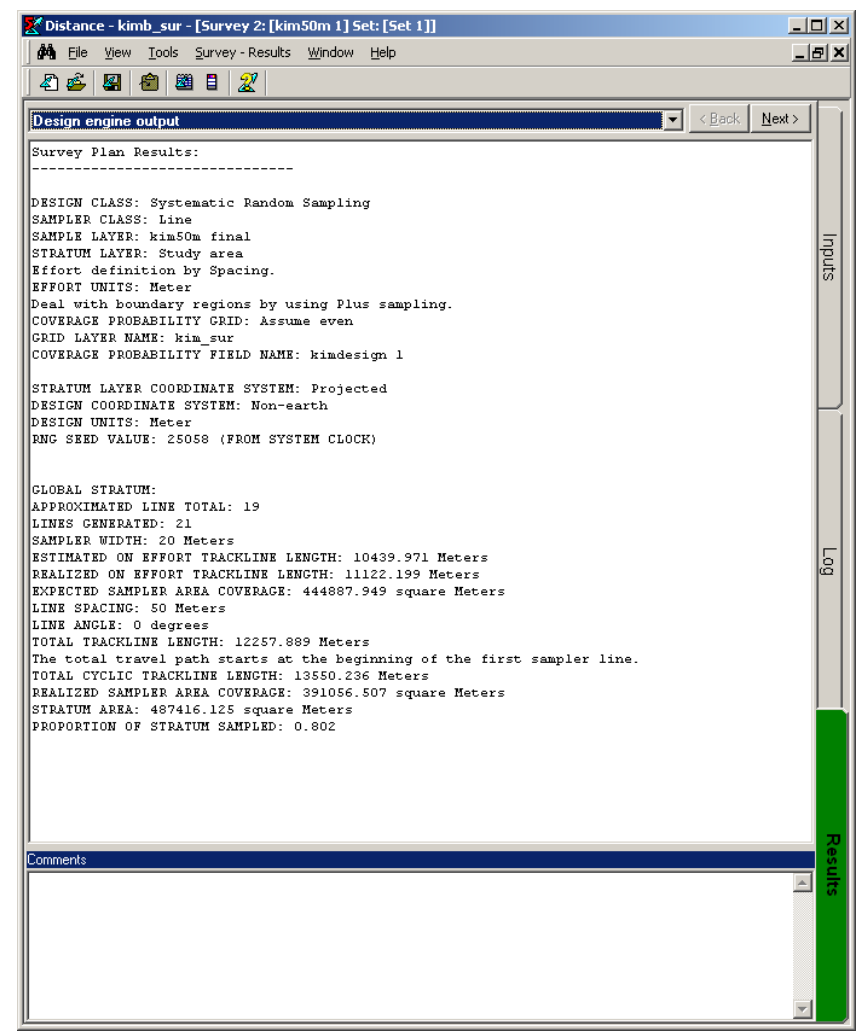

Figure A16. Survey results and map of transects to be surveyed. 


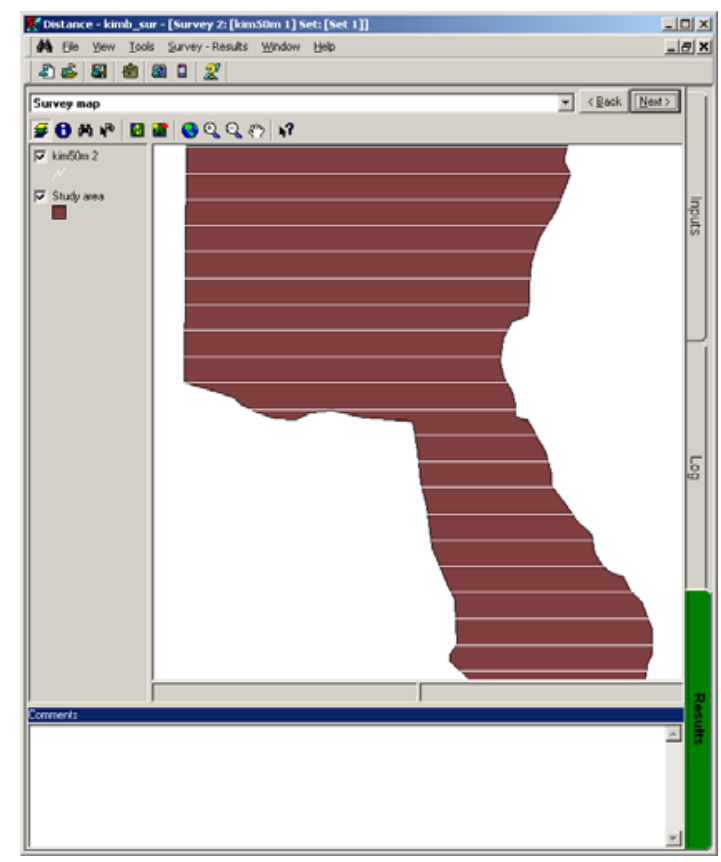

Figure A17. Distribution of the 21 transects selected within suitable tortoise habitat.

Figure A18 identifies the start and end point coordinates of transect. The coordinates listed were formatted as XY coordinates and were downloaded to a GPS unit as waypoints. The 21 transects were then surveyed using LTDS methods, in which 36 tortoises were observed.

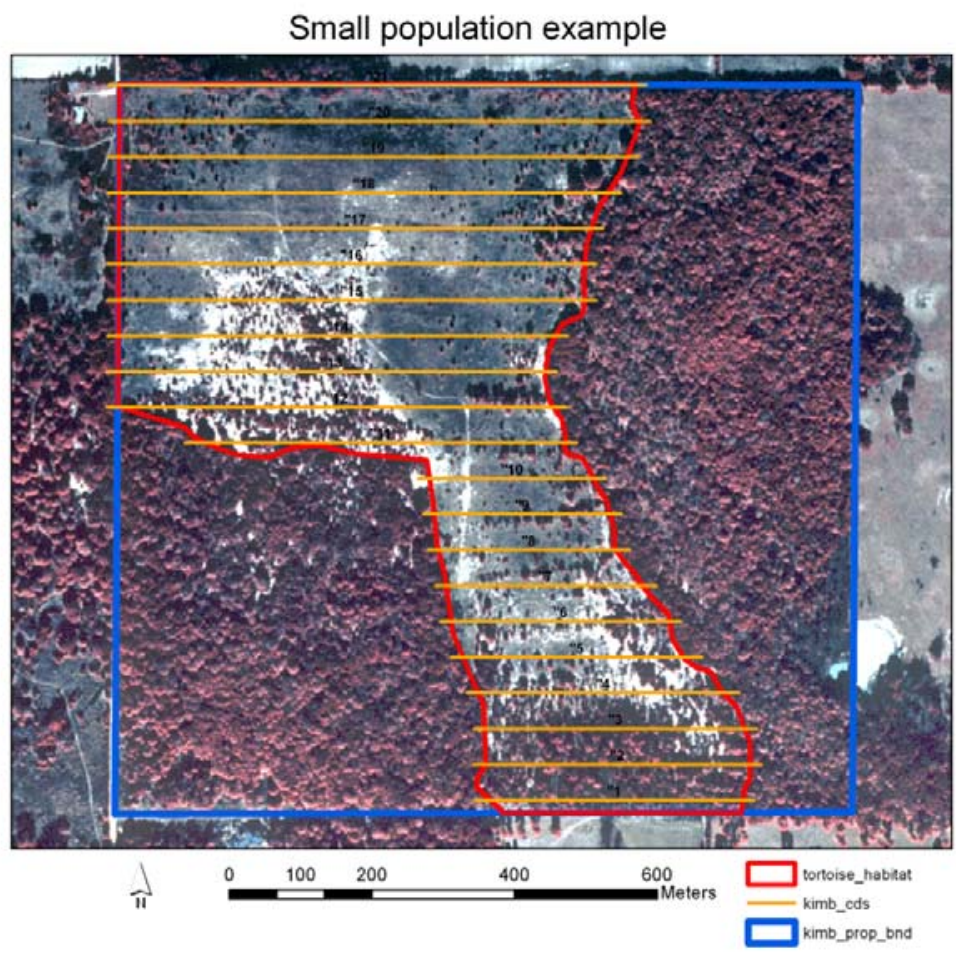

Figure A18. Start and end point coordinates of transect. 
Due to the small size of this population (36 observations), additional sampling was needed to obtain a more precise estimate of the population size (with a CV as close to 20 percent as possible). Sampling options for small populations include: sampling two sets of perpendicular transects or surveying the same transects twice. Assuming the population is closed (no immigration or emigration), sampling additional transects will increase the sample size and allow a more robust estimate to be calculated (Figure A19). After the initial survey, the site was re-sampled using perpendicular transects, which covered $>9 \mathrm{~km}$ of transect, and which resulted in observations of 27 tortoises. Combining results of the two surveys enabled us to derive the population estimate below. If the precision of this estimate was not adequate for the survey goals, additional surveys could have been conducted to derive more precise estimates.

\section{Creation of a distance project (data import)}

After downloading Program Distance from the web site, you will want to create a project. Use Excel or ArcGIS or another data management program to store and manipulate data (Figure A20). (Program Distance is not formatted for data manipulation.)

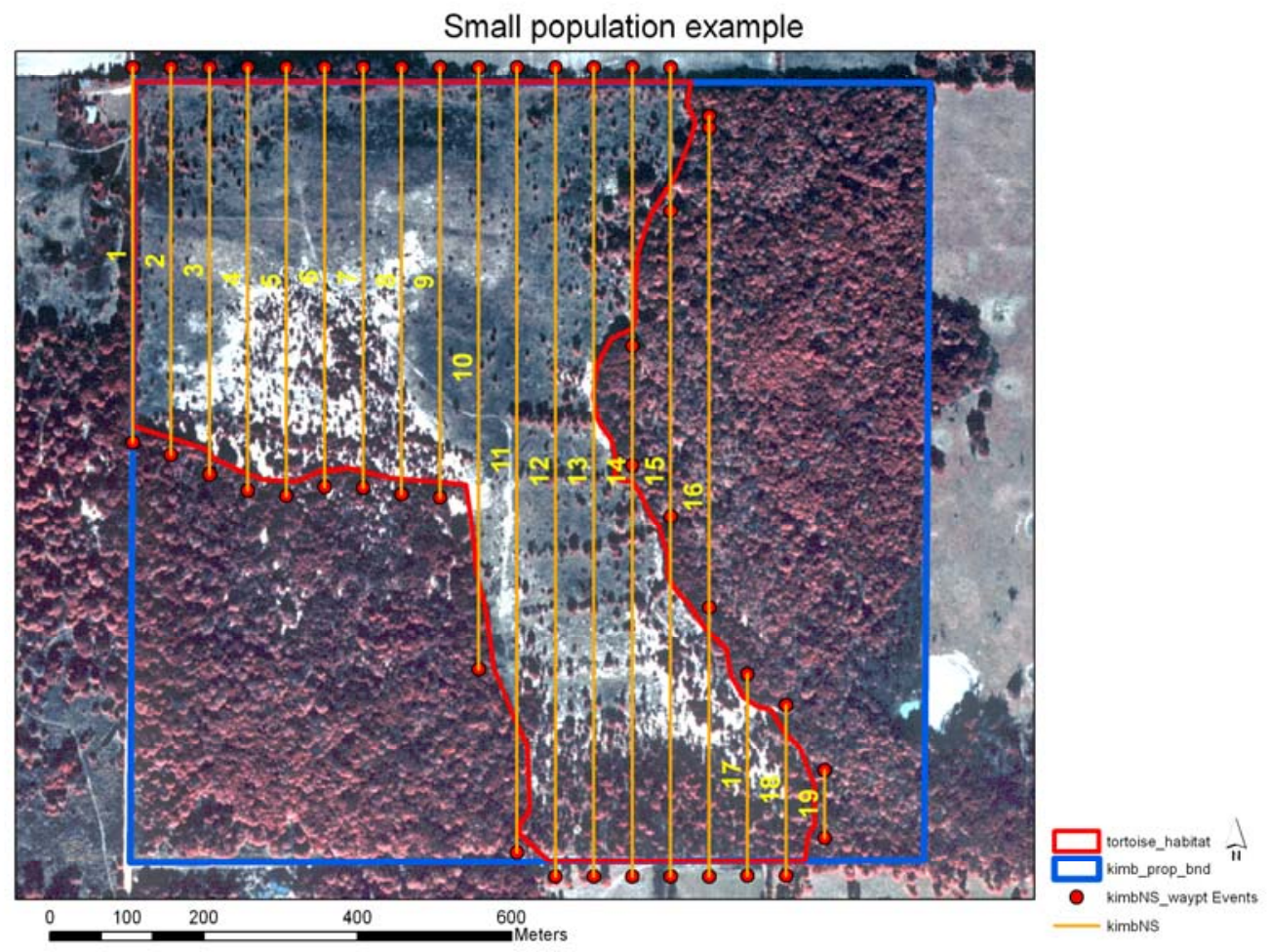

Figure A19. Additional transects placed perpendicular to the previous survey. 


\begin{tabular}{|c|c|c|c|c|c|c|c|c|}
\hline \multicolumn{8}{|c|}{ 区 Microsoft Excel - kimb_tort36.csy } & $-[\square$ \\
\hline \multicolumn{2}{|c|}{ File Edit } & View Inse & ert Format & Iools & windo & Help & Adobe PDF & $\begin{array}{r}-8 \\
x\end{array}$ \\
\hline \multirow{2}{*}{\multicolumn{3}{|c|}{ 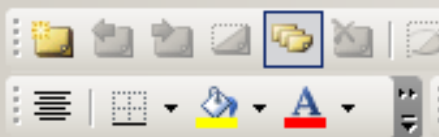 }} & $5 x \mid 2$ & 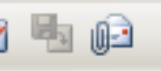 & & \multicolumn{3}{|c|}{ 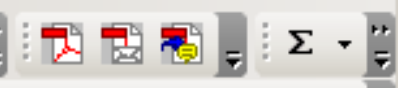 } \\
\hline & & & 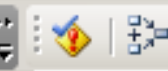 & 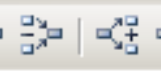 & $x_{\square}\left|O_{i}\right|$ & \multicolumn{3}{|c|}{ 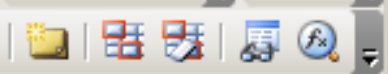 } \\
\hline \multicolumn{2}{|c|}{$\mathrm{A} 6$} & $-\quad y$ & $f_{x}$ & & & & & \\
\hline & $\mathrm{A}$ & $\mathrm{B}$ & $\mathrm{C}$ & $\mathrm{D}$ & $E$ & $F$ & $G$ & $\overline{-1}$ \\
\hline 1 & label & area & label & length & distance & count & label & \\
\hline 2 & small_pop & 48.7416 & 21 & 736 & & \multicolumn{3}{|c|}{0} \\
\hline 3 & & & 20 & 728 & 16 & \multicolumn{3}{|c|}{$1 \mathrm{~A}$} \\
\hline 4 & & & 19 & 650 & 5.6 & \multicolumn{3}{|c|}{$1 \mathrm{~A}$} \\
\hline 5 & & & 18 & 550 & 2.1 & \multicolumn{3}{|c|}{$1 \mathrm{~A}$} \\
\hline 6 & & & 17 & 451 & 34 & \multicolumn{3}{|c|}{$1 \mathrm{~A}$} \\
\hline 7 & & & & & 35 & \multicolumn{3}{|c|}{$1 \mathrm{~A}$} \\
\hline 8 & & & 16 & 611 & 41 & \multicolumn{3}{|c|}{$1 \mathrm{~A}$} \\
\hline 9 & & & & & 3.2 & \multicolumn{3}{|c|}{$1 \mathrm{~A}$} \\
\hline 10 & & & 15 & 652 & 0.5 & \multicolumn{3}{|c|}{$1 \mathrm{~A}$} \\
\hline 11 & & & & & 11.1 & \multicolumn{3}{|c|}{$1 \mathrm{~A}$} \\
\hline 12 & & & & & 15.8 & \multicolumn{2}{|r|}{11} & $\nabla$ \\
\hline \multicolumn{3}{|c|}{$|14 \cdot M|$ kimb_tort36 } & \multicolumn{3}{|r|}{$|1|^{\cdots}$} & \multicolumn{3}{|r|}{.11} \\
\hline \multicolumn{3}{|c|}{ Ready } & & & & \multicolumn{2}{|c|}{ NUM } & \\
\hline
\end{tabular}

Figure A20. Sample project data spreadsheet.

Convert files stored in Excel to *.csv files for import into Distance, or, if you are working in ArcGIS, simply export the ArcGIS data. In the most basic survey, you will have three tiers of information:

1. Region defines the tract name and the area of the sample frame

2. Transects identification and length of transect sampled

3. Observations for each transect with the perpendicular distance, tortoises found, and status information.

Open Program Distance. Under "File," create a new project. (Consult the Help or Wizard for guidance.) Create a template as seen below, and then import the data using the Import Data Wizard (located under "Tools"). You may also use the example as your template.

Figure A21 shows a template that can accept the data in the *.csv file above.

Work your way through the wizard following directions and import the data into Distance (Figure A22). Once the data have been imported, data can be analyzed as follows. 


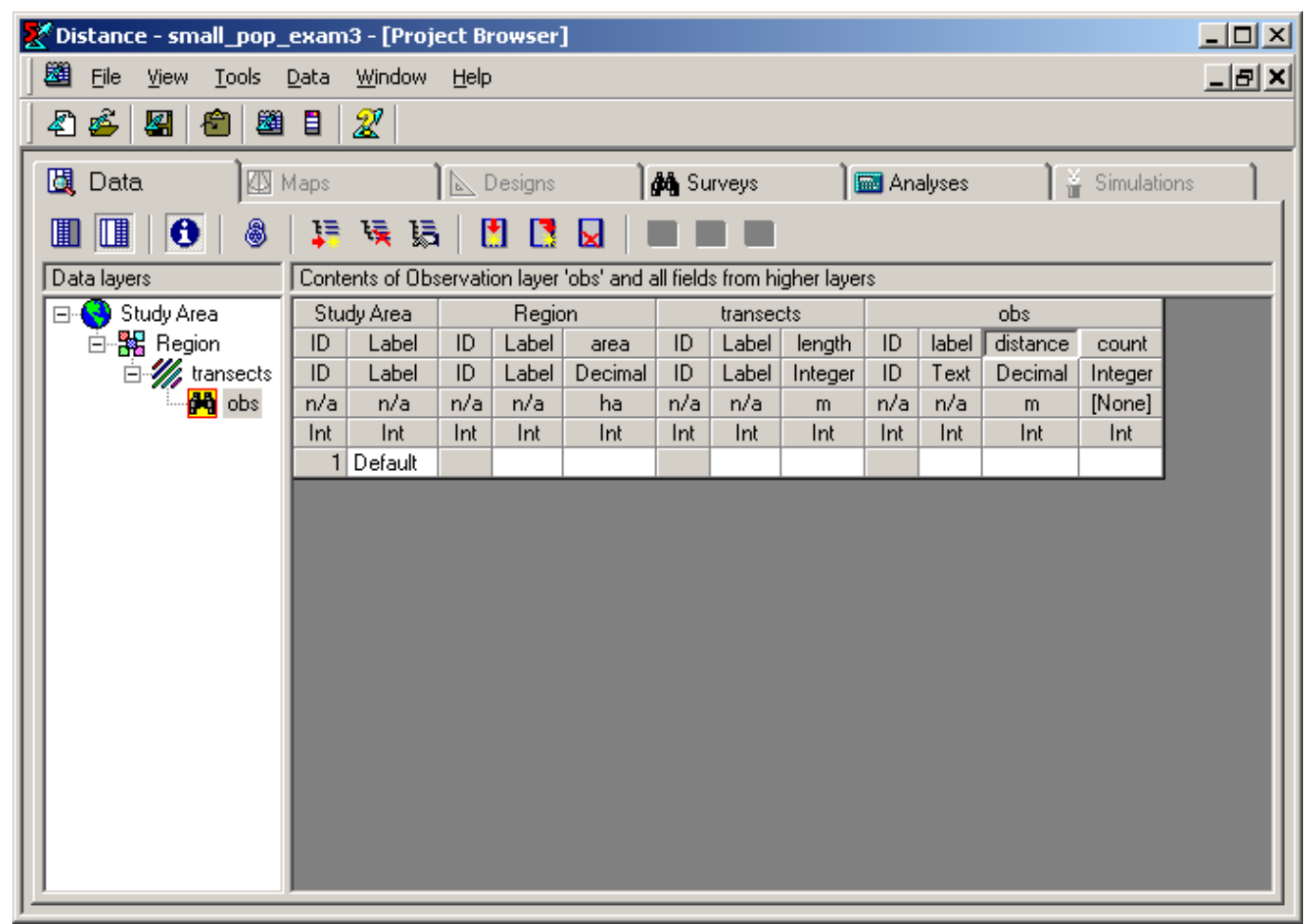

Figure A21. Sample Program Distance template.

\begin{tabular}{|c|c|c|c|c|c|c|c|c|c|c|c|c|c|}
\hline \multicolumn{13}{|c|}{ 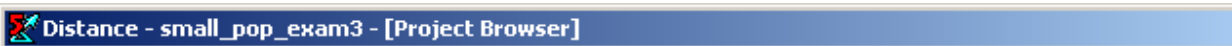 } & $-|\square| x$ \\
\hline \multicolumn{13}{|c|}{ 第 File View Iools Data window Help } & \multirow[t]{2}{*}{$-\underline{a} \underline{\underline{x}}$} \\
\hline \multicolumn{13}{|c|}{ 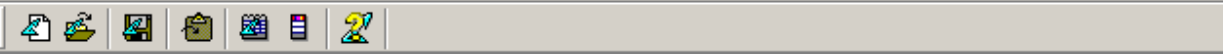 } & \\
\hline \multicolumn{3}{|c|}{ 固 Data } & \multicolumn{2}{|c|}{$\triangle$ Designs } & \multicolumn{3}{|c|}{ Surveys } & \multicolumn{3}{|c|}{ Analyses } & \multicolumn{2}{|c|}{ ii Simulations } & \\
\hline 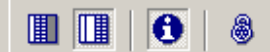 & & 绿 嘼 & & $\square$ & \multicolumn{3}{|c|}{$\square \square \square$} & & & & & & \\
\hline \multicolumn{14}{|c|}{ Data layers Contents of Observation layer 'obs' and all fields f Append New Record After Current } \\
\hline \multirow{20}{*}{ 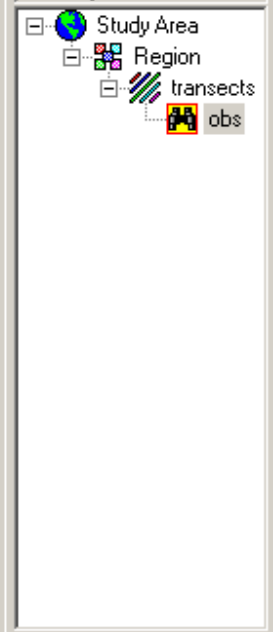 } & \multicolumn{2}{|c|}{ Study Area } & \multicolumn{3}{|c|}{ Region } & \multicolumn{3}{|c|}{ transects } & \multicolumn{4}{|c|}{ obs } & \multirow{20}{*}{$\Delta$} \\
\hline & ID & Label & ID & Label & area & ID & Label & length & ID & label & distance & count & \\
\hline & ID & Label & ID & Label & Decimal & ID & Label & Integer & ID & Text & Decimal & Integer & \\
\hline & $n / a$ & $n / a$ & $\mathrm{n} / \mathrm{a}$ & $n / a$ & ha & $n / a$ & $\mathrm{n} / \mathrm{a}$ & $\mathrm{m}$ & n/a & $\mathrm{n} / \mathrm{a}$ & $\mathrm{m}$ & [None] & \\
\hline & Int & Int & Int & Int & Int & Int & Int & Int & Int & Int & Int & Int & \\
\hline & & \multirow{15}{*}{ Default } & & \multirow{15}{*}{ kimbrel } & \multirow{15}{*}{48.7416} & & 21 & 736 & 1 & & 0 & 0 & \\
\hline & & & & & & & 20 & 728 & & A & 16 & 1 & \\
\hline & & & & & & & 19 & 650 & & A & 5.6 & 1 & \\
\hline & & & & & & & 18 & 550 & & A & 2.1 & 1 & \\
\hline & & & & & & & 17 & \multirow{2}{*}{451} & & A & 34 & 1 & \\
\hline & & & & & & & 18 & & & A & 35 & 1 & \\
\hline & & & & & & \multirow{2}{*}{\multicolumn{2}{|c|}{616}} & \multirow{3}{*}{611} & & A & 41 & 1 & \\
\hline & & & & & & & & & & A & 3.2 & 1 & \\
\hline & & & & & & \multirow{3}{*}{\multicolumn{2}{|c|}{715}} & & & A & 0.5 & 1 & \\
\hline & & & & & & & & \multirow[t]{2}{*}{652} & & A & 11.1 & 1 & \\
\hline & & & & & & & & & 11 & & 15.8 & 1 & \\
\hline & & & & & & & & & & A & 11.1 & 1 & \\
\hline & & & & & & & 14 & 599 & 13 & A & 4.2 & 1 & \\
\hline & & & & & & & & 505 & 14 & & 12.6 & 1 & \\
\hline & & & & & & & & & 15 & A & 5 & 1 & \\
\hline
\end{tabular}

Figure A22. Sample Program Distance data analysis. 


\section{Summary and analysis of the data}

The goal of distance sampling is to estimate the density (D) and abundance $(\mathrm{N})$ of tortoises over a defined area (A). Several tests are available in Distance to determine the model that best fits the distribution of your data. Initially, you should use your raw data without any constraints to be sure the data can be imported into the program, to determine whether any assumptions have been violated, and to examine the distribution of the data. Assuming that the data have been successfully imported and none of the assumptions of the model have been violated, an examination of the distribution of the data is needed to determine where it should be "truncated" (w). Simply put, truncation is putting a constraint on your transect width to eliminate outlying observations. Outliers do not add much information to the model you are creating and often make it difficult to fit the data to a model.

Typically, the most distant observations (5 percent) are removed from the data set as outliers. However, the distribution of the data should be reviewed to determine if there is possibly another clear break in the data where w should be chosen. Alternatively, data collection can be truncated in the field using a fixed transect width that eliminates outlying observations. However, you should only do this if you are certain you will have a sufficient number of detections (at least $60-80$ tortoises).

There are no cookbook methods to evaluate and definitively determine the best model for your dataset. Analysis must be initiated by running several different models, with adjustment terms, in different combinations to determine the model that best fits the data. Using Akaike's information criterion (AIC), which is a measure of the goodness of fit of an estimated statistical model, you will determine the model that best fits your data. For tortoise data you will typically be using a uniform, hazard-rate or half-normal model with one of the three series adjustments (cosine, simple polynomial, hermite polynomial). To determine the model that best fits your data, you must evaluate AIC values, QQ plots, Kolmogorov-Smirnov, and Cramervon Mises tests to help choose the most appropriate model.

When sample size (n) is low, use AICc. Models that are within about two AIC units are essentially equivalent. AIC CANNOT be used for model selection if data sets are different, for example:

1. Sample size differs (i.e., one analysis $n=60$ and another $n=65$ ),

2. Truncation distance $(\mathrm{w})$ differs among transects, 
3. Data are grouped or cutpoints differ, or

4. Data are grouped in one analysis and ungrouped in the other.

Since tortoise data consists of individual measured distances, goodness of fit tests are not a useful metric. Models that are within about two AIC units are essentially equivalent. The data analysis that follows includes some general guidelines.

Go to the analysis tab and accept all defaults to run the data (Figure A23). Do not use a data filter and do not stratify the analysis. This will give you a clear view of the data distribution and examine if any assumptions have been violated. Review assumptions of LTDS above.

Do not stratify the analysis (i.e., do not click on the "transform distance data into intervals for analysis" button, Figure A24). Run the analysis with the defaults and examine the results. Go to the results tab and look at the summary (Figure A25).

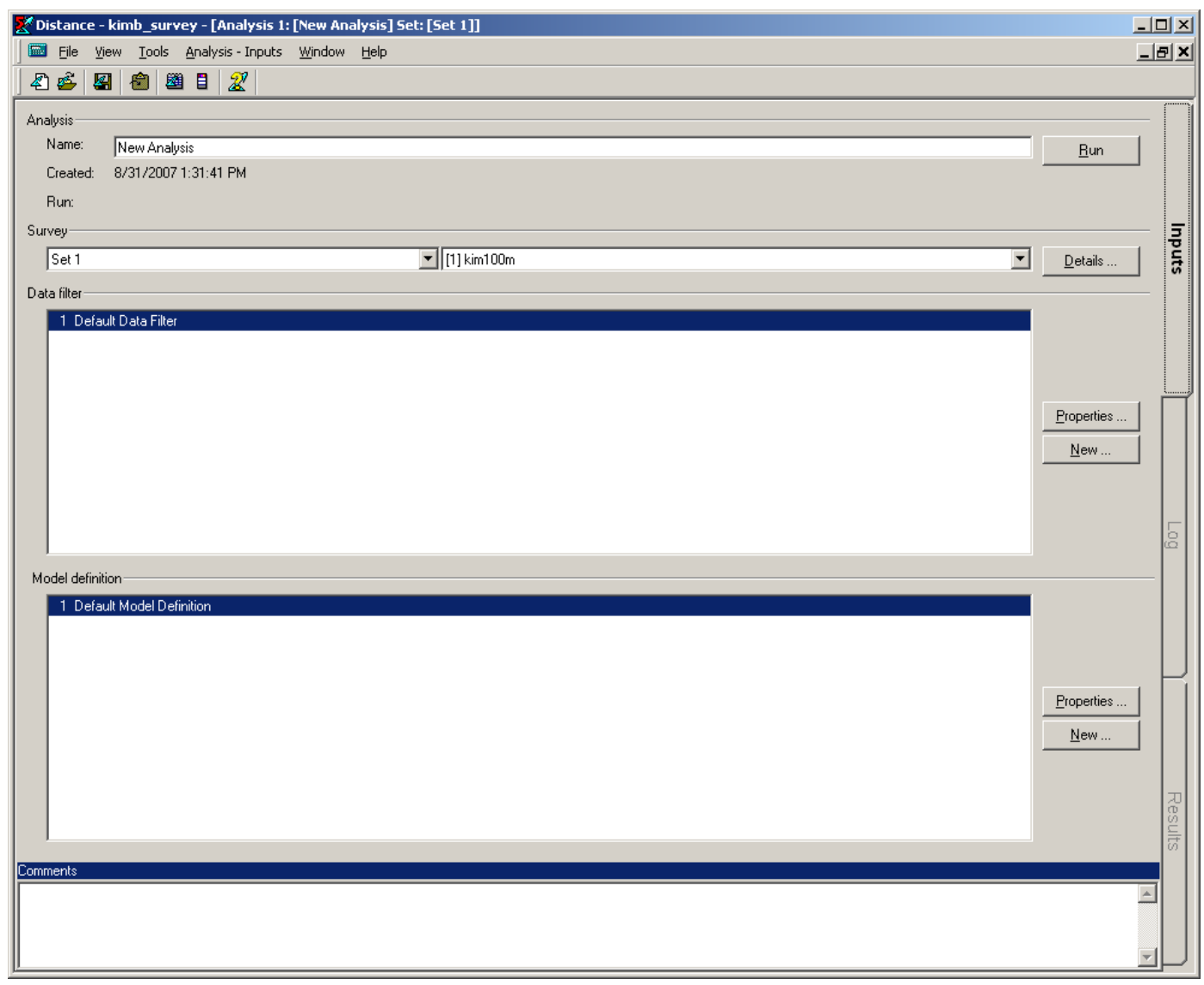

Figure A23. Analysis of individual measured distances data. 


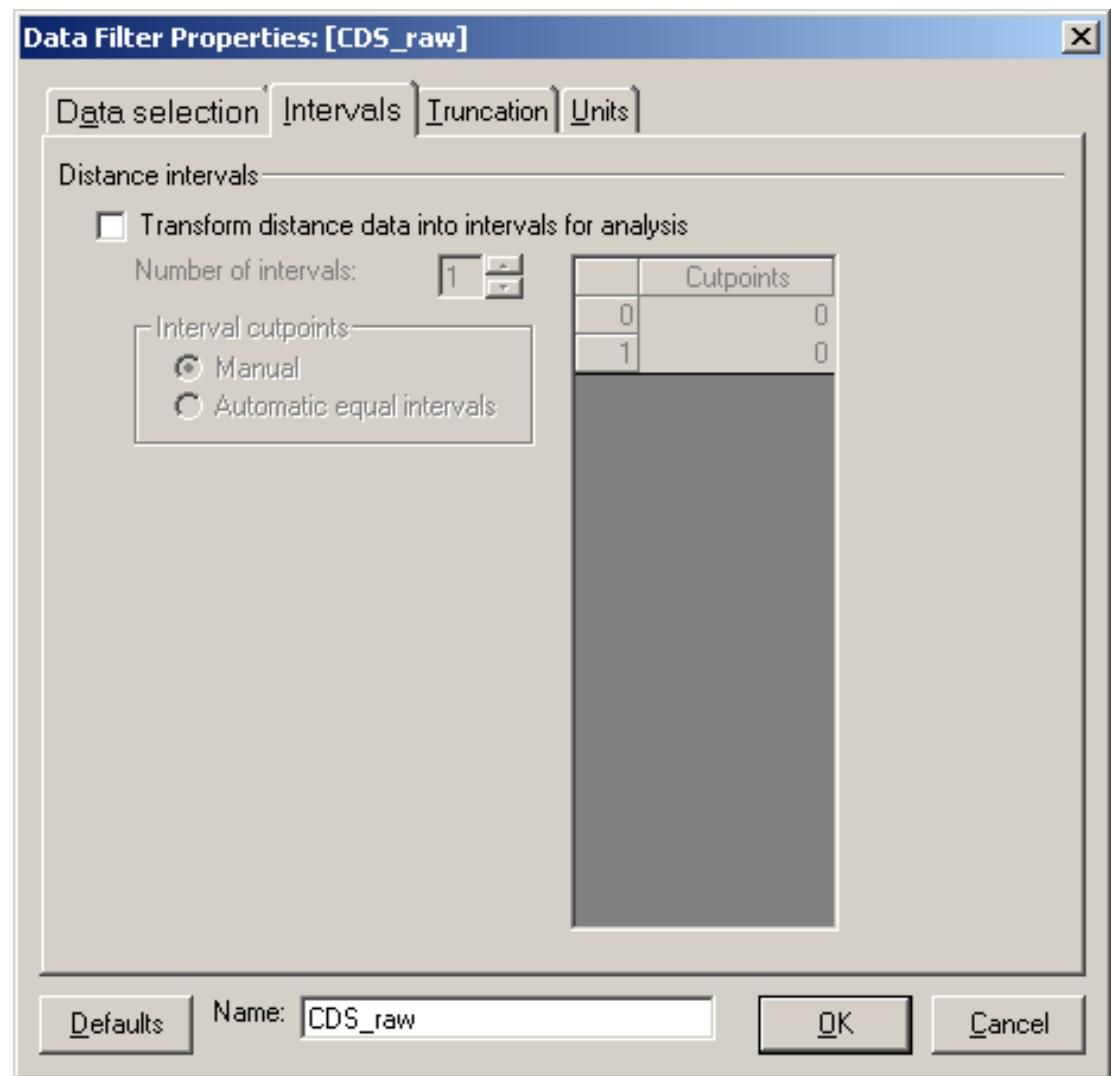

Figure A24. "Intervals" tab- do not click on "transform distance data into intervals for analysis" checkbox.

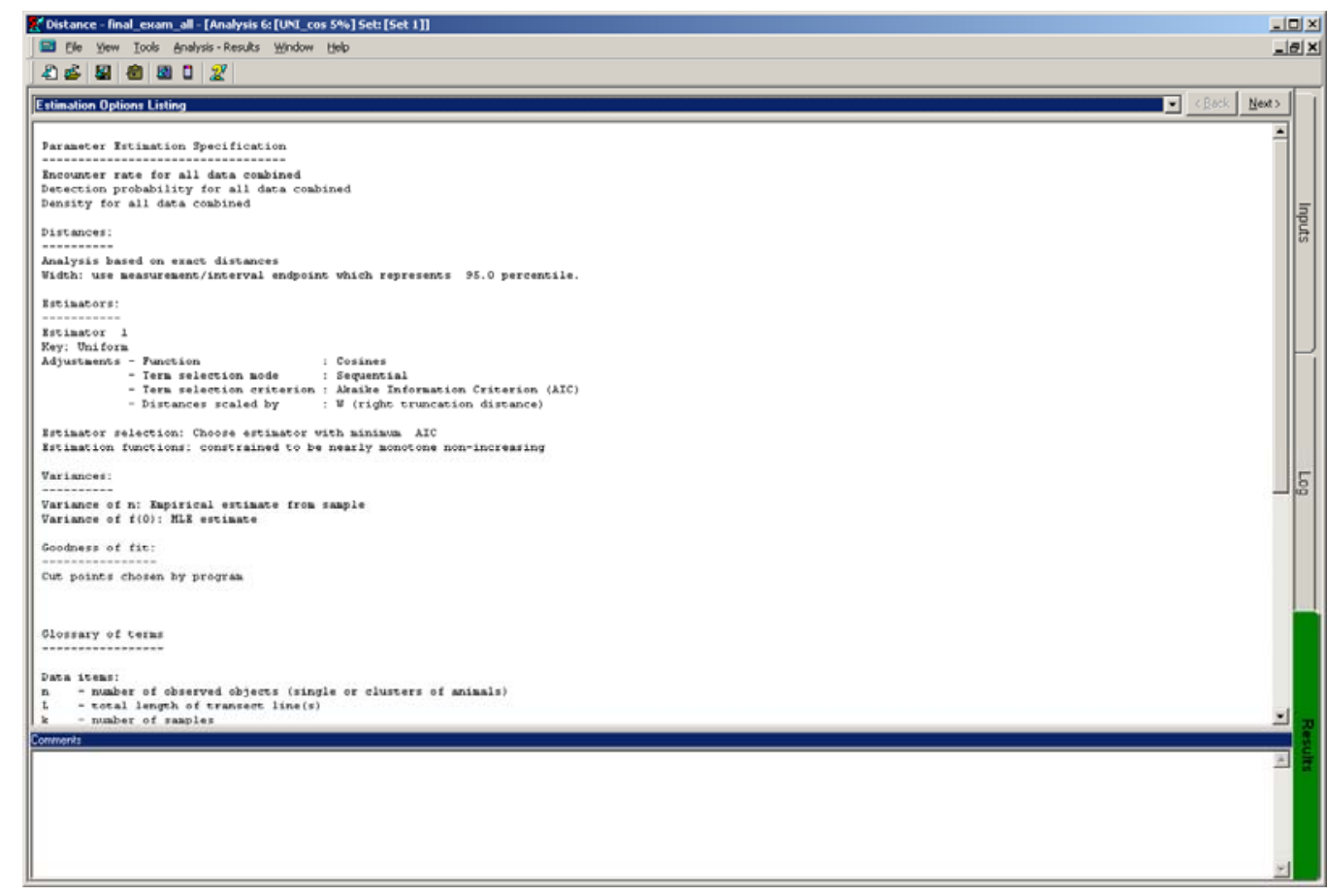

Figure A25. Results of individual measured distances data analysis. 
The Estimating Options Listing (Figure A26) includes a list of the models being tested and a glossary of terms and abbreviations found in the output.

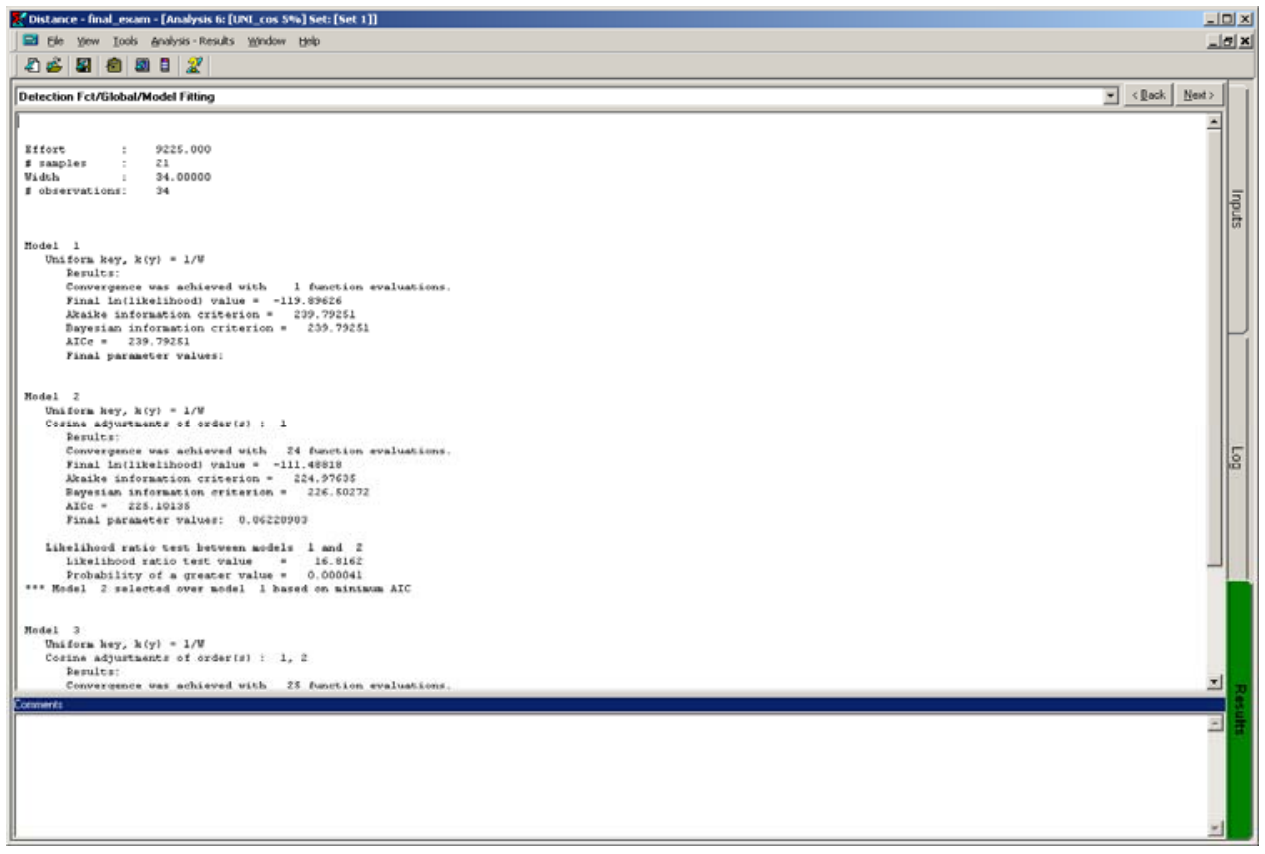

Figure A26. Estimating options listing.

The second page, Detection Fct/Golbal/Model Fitting (Figure A27) displays an evaluation of the model used to fit the distribution of the data and the relative AIC values along with the model that was selected.

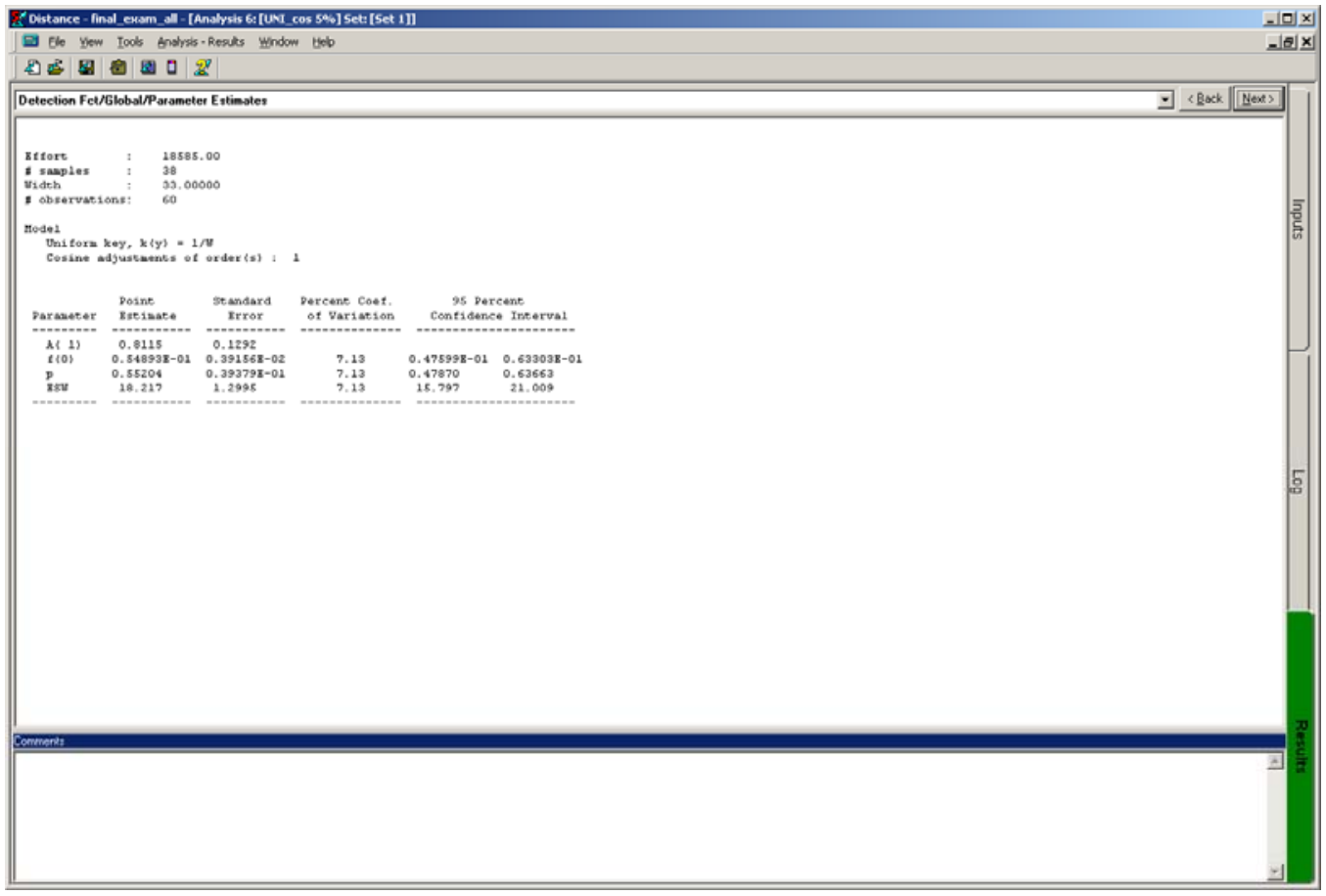

Figure A27. Detection Fct/Golbal/Model output. 
The Detection Fct/Global Estimates page lists results including the total length of transect sampled, number of transects, width of the transects, number of observations, and the model that was selected. The $\mathrm{f}(\mathrm{o})$ is the "fitted probability density function" (Figure A28), which is evaluated at zero distance from the center-line. The variable " $p$ " is the probability that a tortoise is detected given that it is somewhere within the area surveyed. The ESW is the effective strip width for all transects.

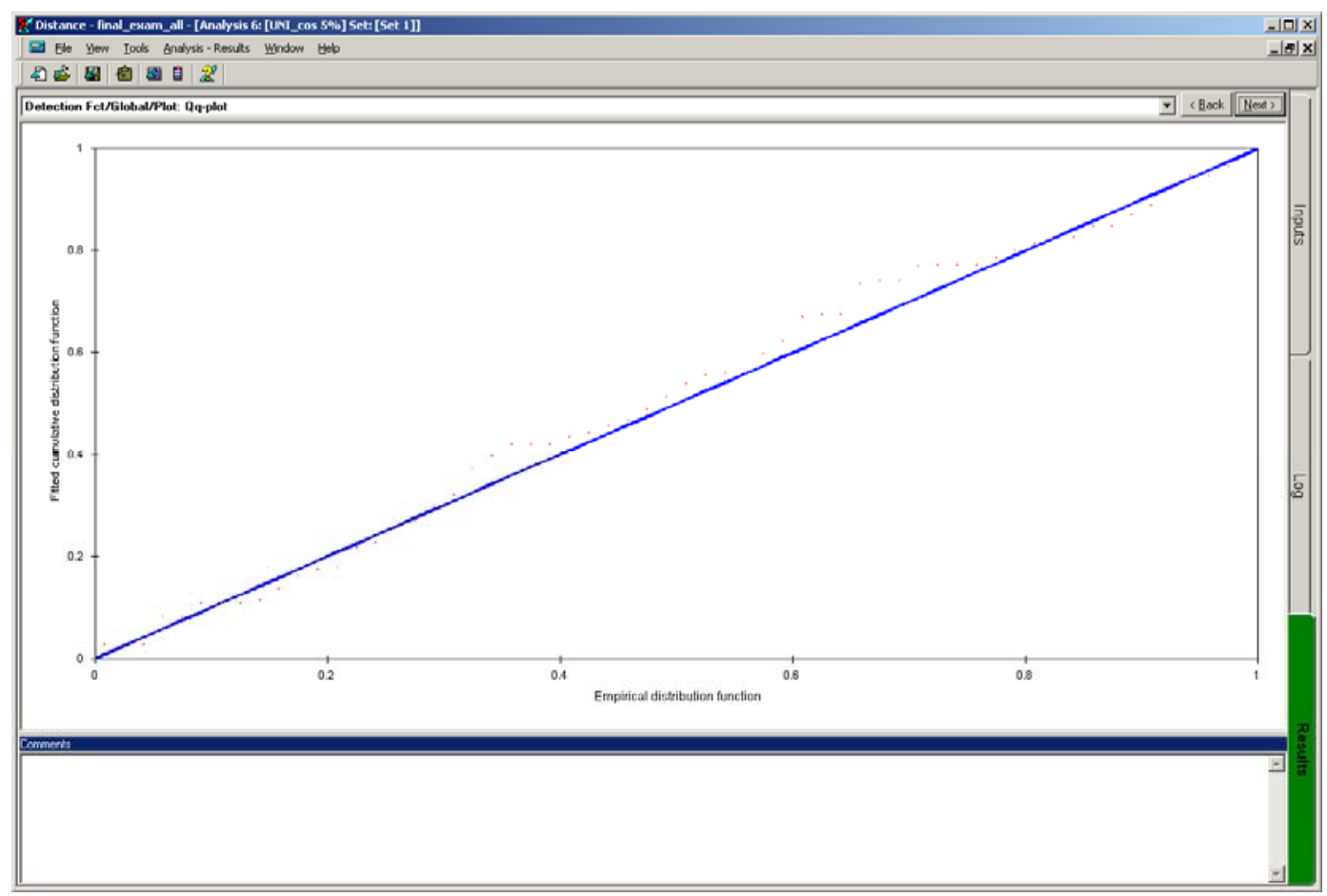

Figure A28. Graph of fitted probability density function.

The QQplot lets you see where there may be pooling in the distribution of the data. Ideally, the data should fall on the line; however, in the real world, the data should be distributed close to or along the line. If the data are pooled, i.e., the points are heaped together rather than falling along the line, you may have chosen the wrong model, or there may be problems with the data.

The Detection Fct/Global/K-S GOF test (Figure A29) assesses whether the model adequately fits the data. The next six pages include graphics of the distribution of the data and their associated GOF tests. GOF test are not a good indicator of model fit since tortoise measures exact distances.

The Density Estimates/Global page you will get the values of encounter rate, density and abundance with standard error, coefficient of variation (\%), and 95 percent confidence intervals. Below you will see a percentage 
breakdown of the variability in density by detection probability and encounter rate. Ideally, you would like to lower detection probability and place more variability in the encounter rate percentage. The survey is conducted with the intent of detecting all the animals.

Several distributions of the data will then be represented with Goodness of Fit results (Figure A30). Check these distributions to determine if there is any heaping in the data.

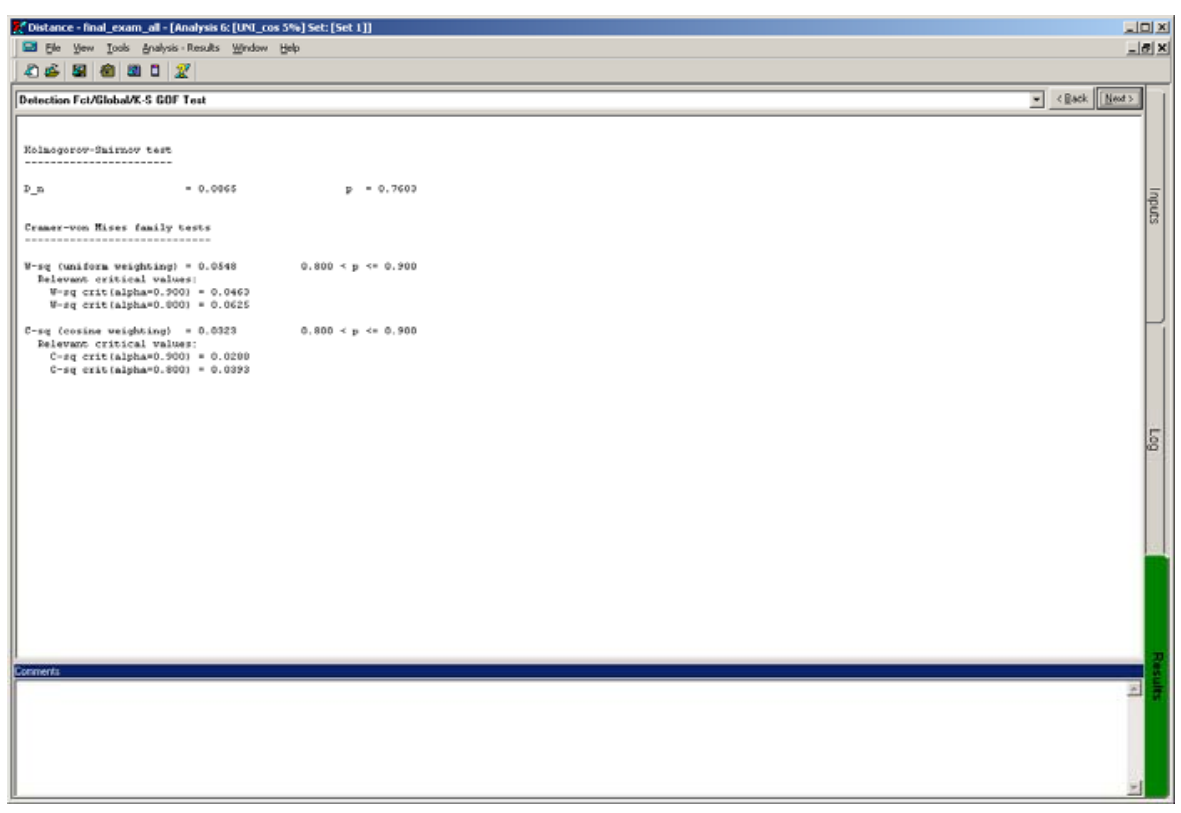

Figure A29. Output of Detection Fct/Global/K-S GOF test.

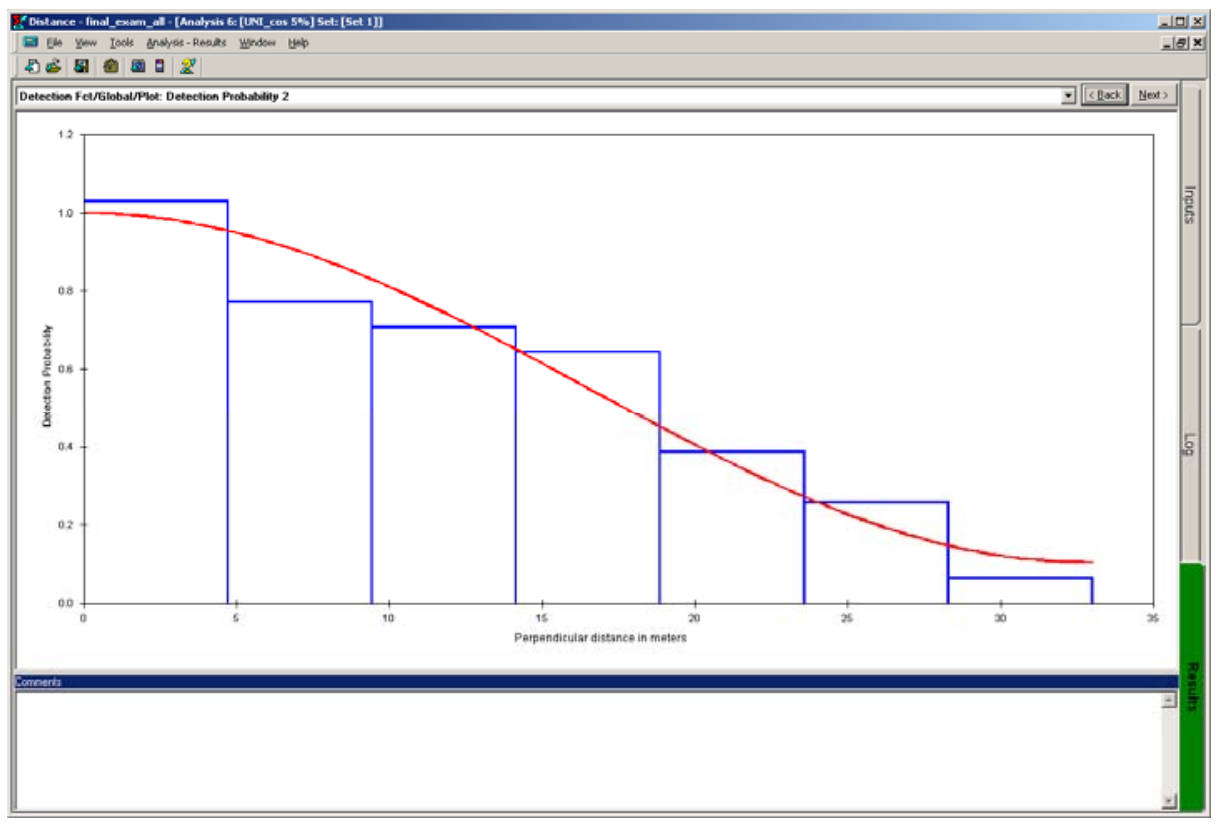

Figure A30. “Goodness of Fit” results. 
The next tab (Figure A31) has information of effort, (n) number of tortoises encountered, $(\mathrm{k})$ number of transects surveyed, (L) total length of the transects, $(\mathrm{n} / \mathrm{L})$ the encounter rate and the width $(\mathrm{w})$ used in the analysis.

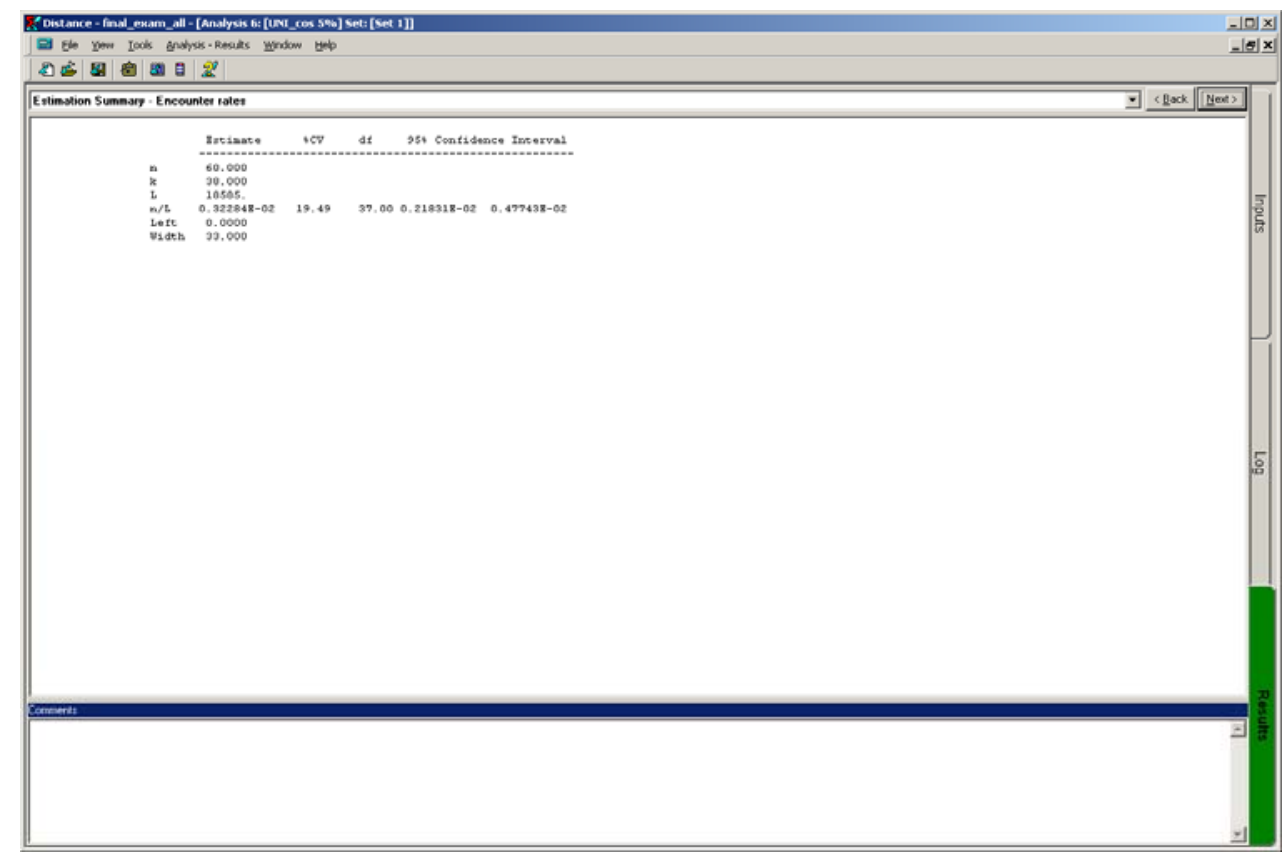

Figure A31. Values for parameters used in the analysis.

The Estimation Summary-Detection probability tab (Figure A32) gives the AIC values for the summary for the chosen model.

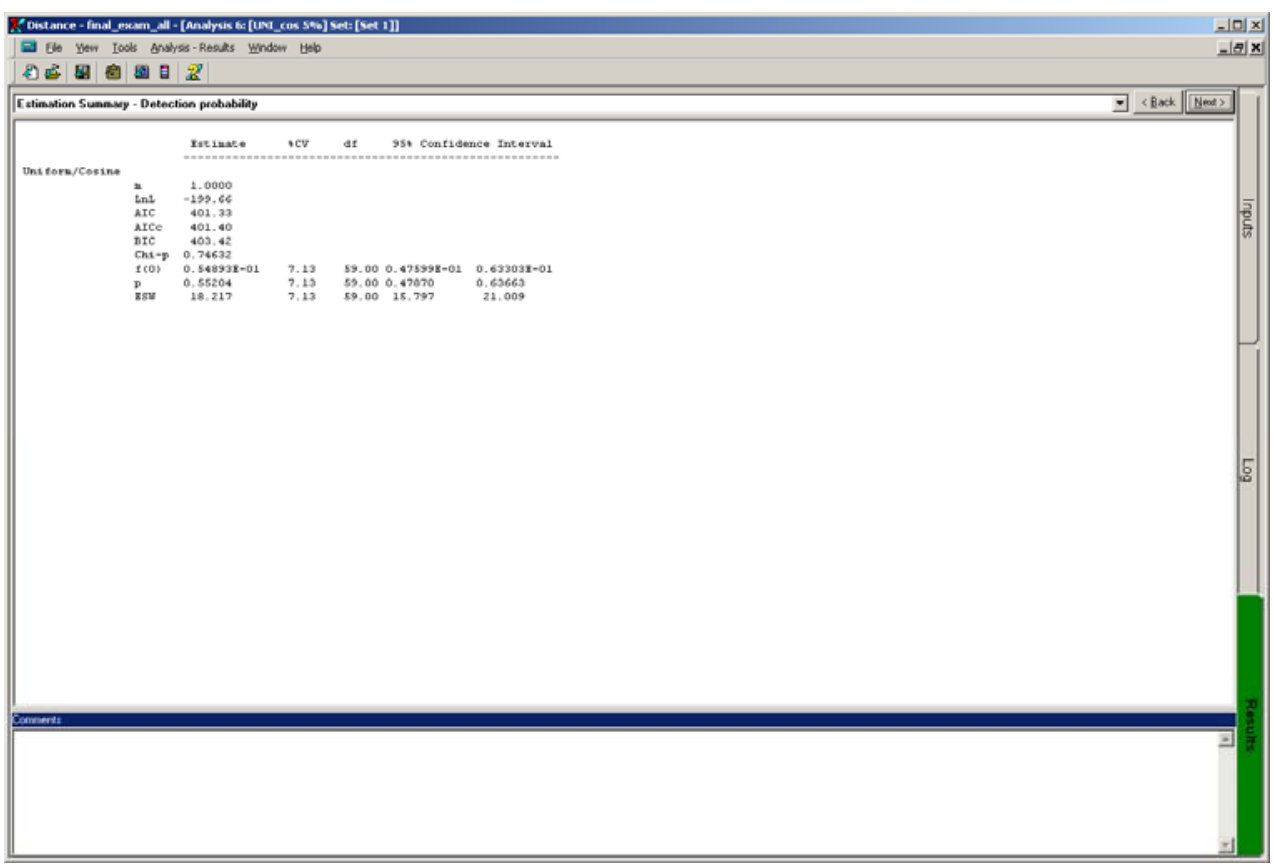

Figure A32. Estimation summary-detection probability tab. 
The final tab gives the estimates for $\mathrm{D}$ and $\mathrm{N}$ for the area surveyed (Figure A33).

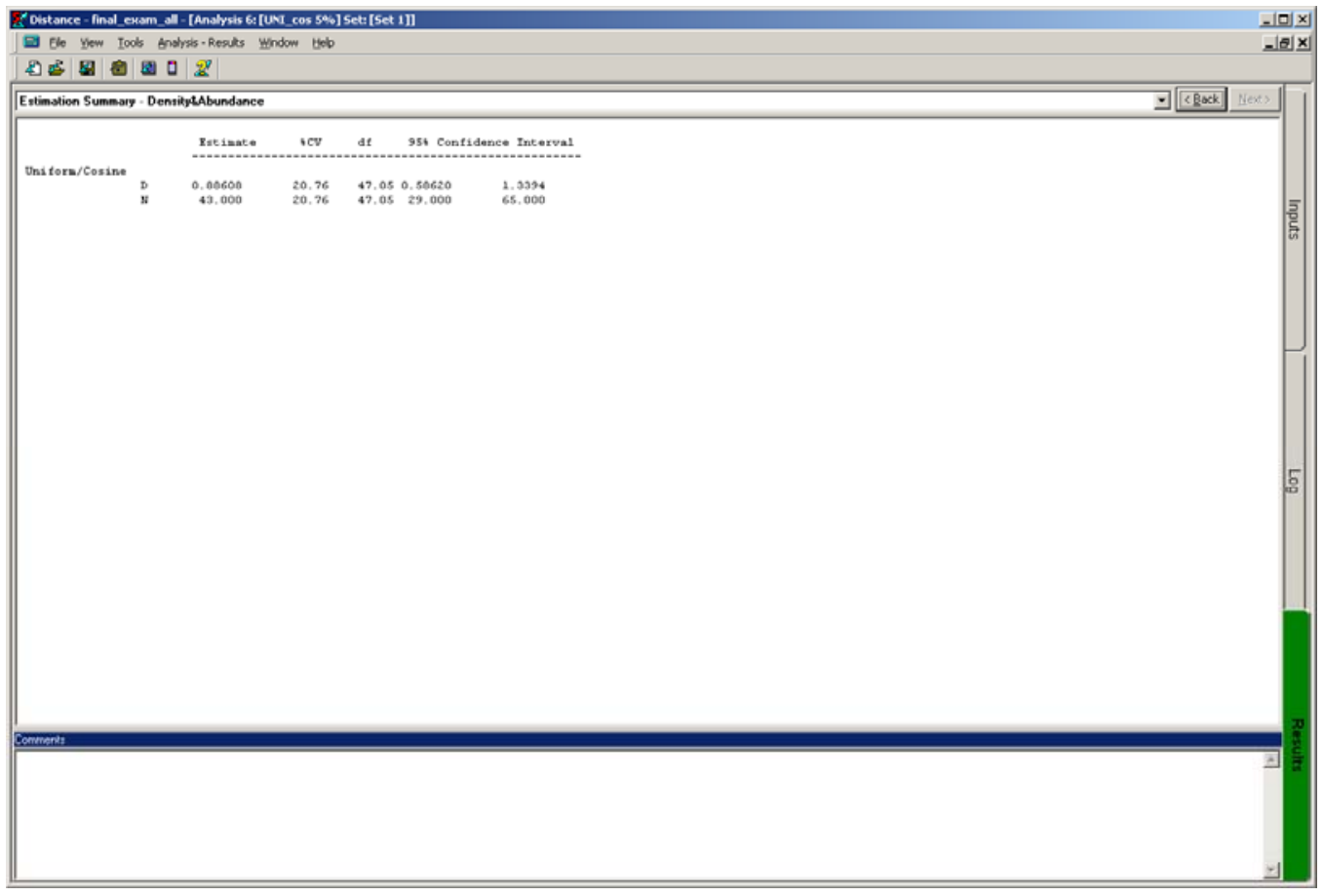

Figure A33. Estimates for $\mathrm{D}$ and $\mathrm{N}$ for the area surveyed.

There are three basic models that you might use to model the distribution of the data: Uniform, Half-normal, and Hazard-Rate. There are adjustment terms that can be used to better fit the models; Cosine, simple polynomial, and hermite polynomial. For most gopher tortoise models, you will be using either uniform or half normal models with a cosine adjustment term. You can place all possible models with the same truncation point and run them all to determine the best model. The naming convention for each model run is the model used, adjustment term, and truncation point for the dataset.

Go back to the analysis main page (Figure A34). Create a new analysis, select the gray ball, and enter the analysis page (Figure A35). 


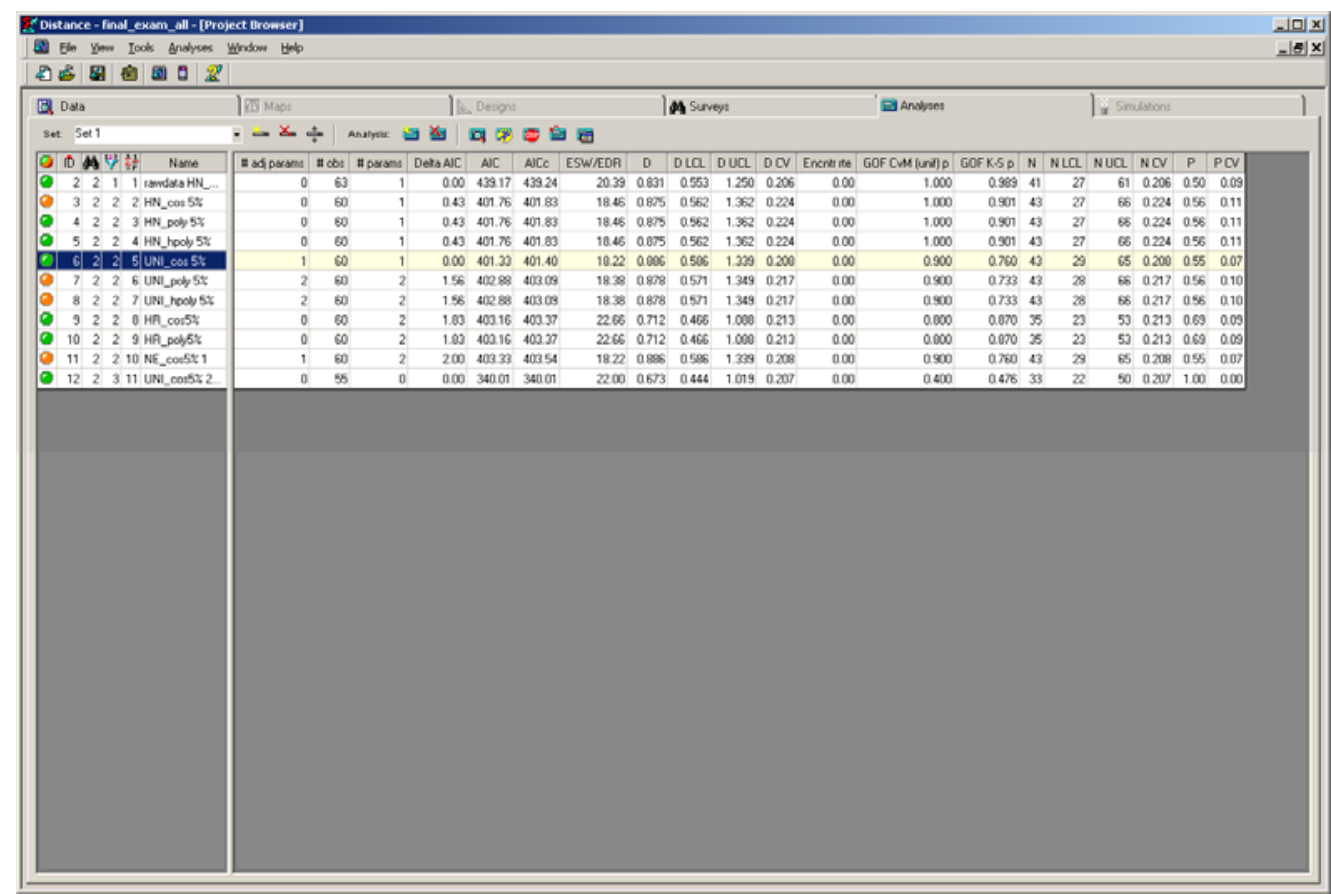

Figure A34. Analysis main page.

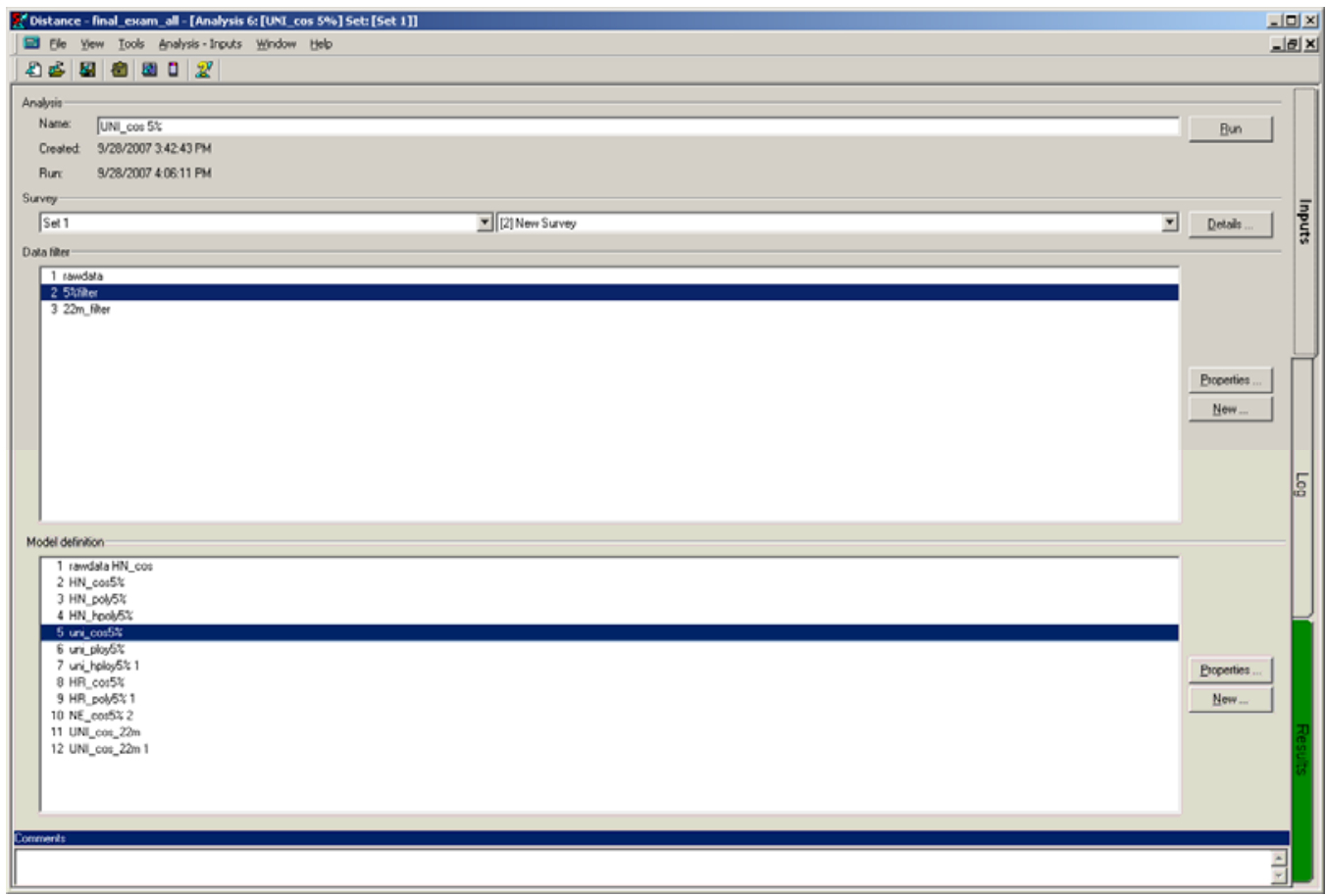

Figure A35. Entering the analysis page.

With 63 observations, a 5 percent cut would reduce the number of observations to 6o. However, depending on distributions of the observations, a different truncation point might be useful. 
Using the data filter above, run one model at a time (Figure A36). You can set up the project to run a list of the most likely models and run them all at once as seen above.

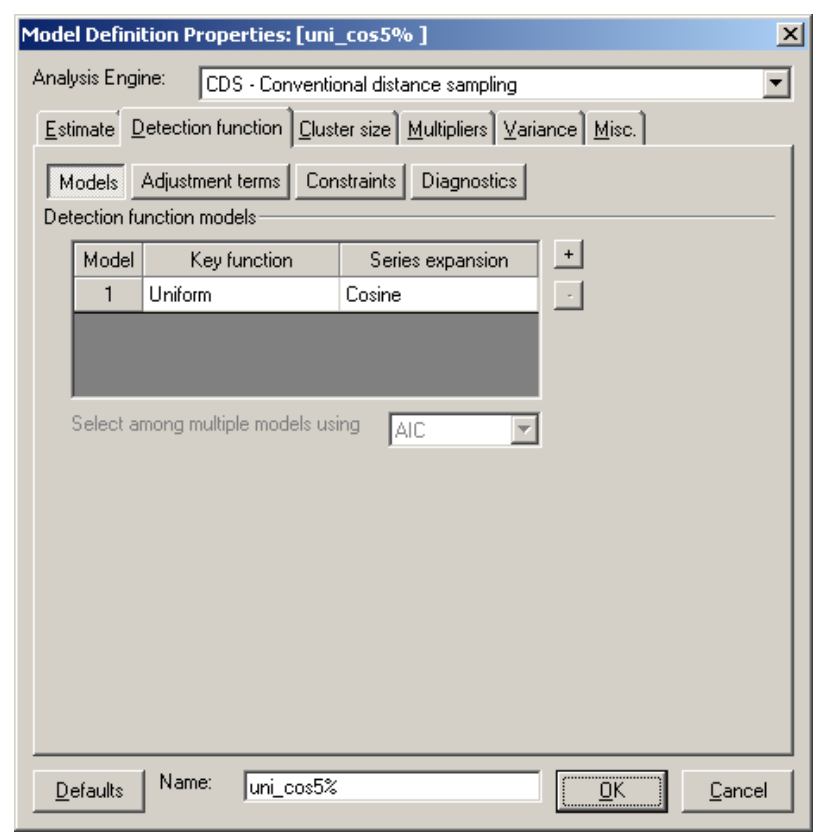

Figure A36. Use the data filter to run one model at a time.

For a small population with a limited sample size, the CV achieved in this example (20.76 percent) is acceptable for long-term population monitoring of population trends (Figure A37).

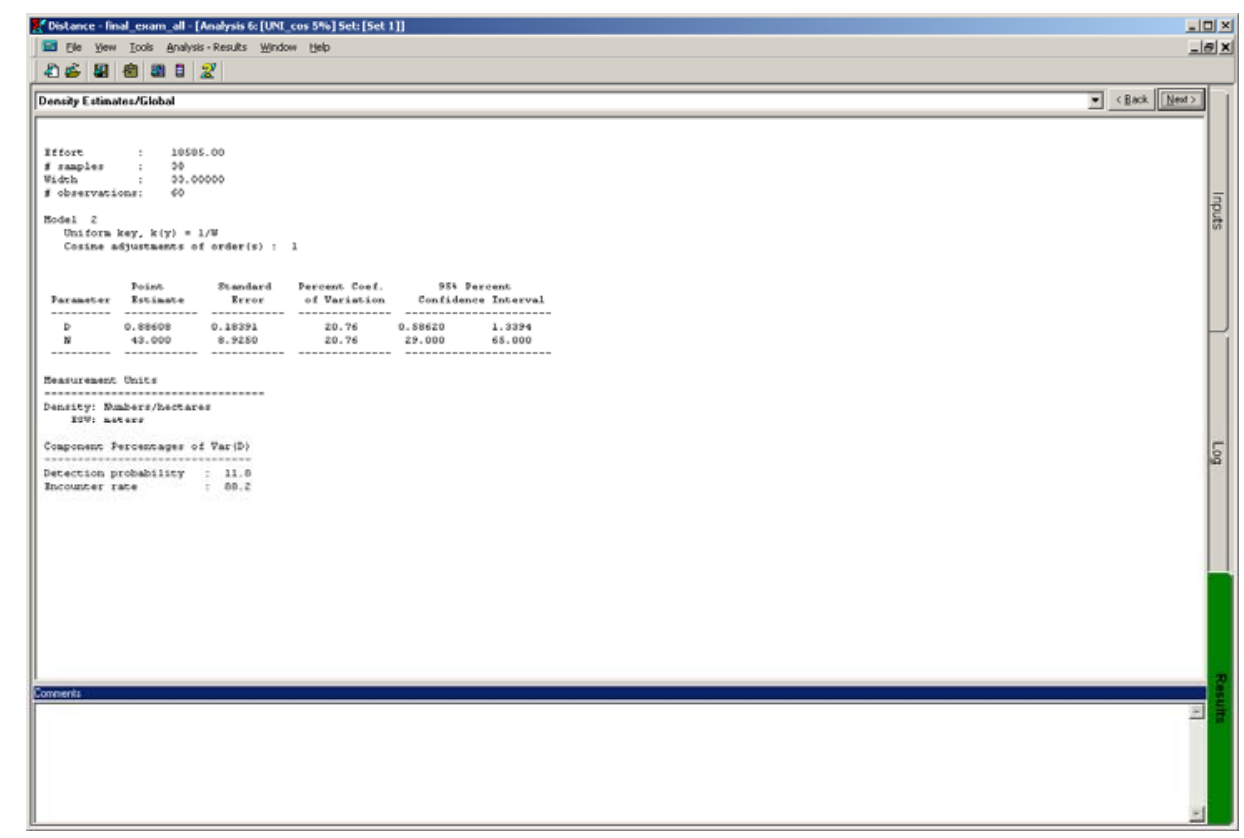

Figure A37. Acceptable CV (20.76\%) for a small population with a limited sample size. 
After running several different models, you will have a table with the results of each model run (Figure A38).

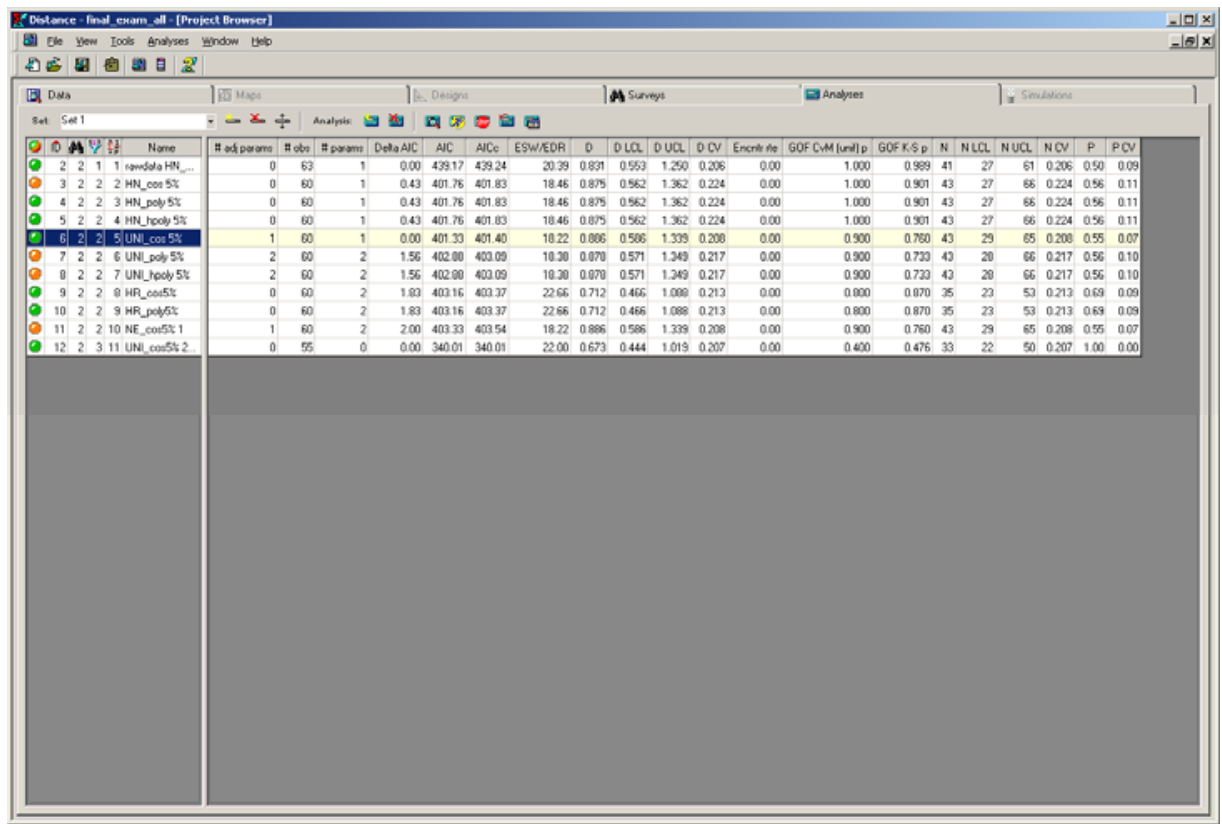

Figure A38. Review the different models on analysis main page.

Review the different models and determine the model that best fits the distribution of distances. Evaluate the AIC values, the number of adjustment parameters, the QQplot, and the GOF test, and determine the model that best fits the data. This will be your population estimate. In this example, all the models gave estimates well within the 95 percent CI for the others. Replicating this survey over time will reveal population trends. See the bootstrap analysis in the manual to determine the model that is best among a group of models with similar AIC values. 


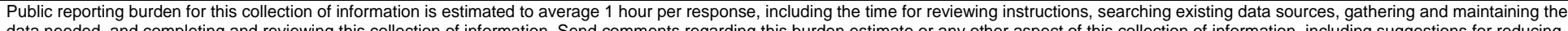

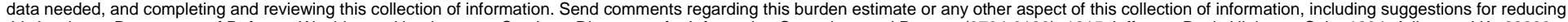

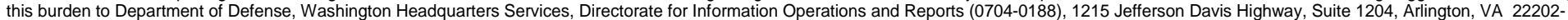

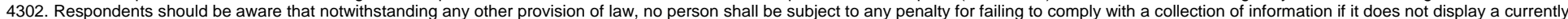
valid OMB control number. PLEASE DO NOT RETURN YOUR FORM TO THE ABOVE ADDRESS.
1. REPORT DATE (DD-MM-YYYY)
2. REPORT TYPE
3. DATES COVERED (From - To)

15-03-2009

Final

4. TITLE AND SUBTITLE

Gopher Tortoise Survey Handbook

5a. CONTRACT NUMBER

5b. GRANT NUMBER

5c. PROGRAM ELEMENT

6. AUTHOR(S)

Lora S. Smith, Jonathan Stober, Harold E. Balbach, and William D. Meyer

5d. PROJECT NUMBER

622720A896

5e. TASK NUMBER

5f. WORK UNIT NUMBER

G9K2FL

7. PERFORMING ORGANIZATION NAME(S) AND ADDRESS(ES)

U.S. Army Engineer Research and Development Center (ERDC)

Construction Engineering Research Laboratory (CERL)

PO Box 9005,

Champaign, IL 61826-9005

8. PERFORMING ORGANIZATION REPORT NUMBER

ERDC/CERL TR-09-7

9. SPONSORING I MONITORING AGENCY NAME(S) AND ADDRESS(ES)

Environmental Division Office

10. SPONSOR/MONITOR'S ACRONYM(S)

600 Army Pentagon

Washington, DC 20310-0600

DAIM-ED

11. SPONSOR/MONITOR'S REPORT NUMBER(S)

12. DISTRIBUTION I AVAILABILITY STATEMENT

Approved for public release; distribution is unlimited.

13. SUPPLEMENTARY NOTES

\section{ABSTRACT}

The gopher tortoise (Gopherus polyphemus) has some legal protection, Federal or state, in all of the states where they occur. Threats to the species vary regionally, and it is often desirable to determine in a statistically acceptable manner whether tortoise populations are being maintained or are declining. There is currently no reliable information on the status and trends of populations across the range, although most studies appear to show that declines are almost universal. Gopher tortoise surveys may be conducted for different purposes, e.g., detection of tortoise presence; estimating population size, density, and trends; and temporal monitoring to assess effects of habitat management or restoration. A survey’s specific objectives determine its timing and method. Survey and population monitoring methods have not been standardized. This makes it difficult to establish reliable gopher tortoise population information because it is not possible to reliably compare reports among different studies. This handbook outlines standardized methods for gopher tortoise surveys, and suggests which methodologies are best applied to each type of need and habitat setting. The conclusion is that Line Transect Distance Sampling is the most statistically reliable when accurate measurement of tortoise population numbers is needed.

\section{SUBJECT TERMS}

gopher tortoise, survey, natural resources management, threatened and endangered species (TES)

\begin{tabular}{|l|c|c|}
\hline \multicolumn{2}{|c|}{ 16. SECURITY CLASSIFICATION OF: } \\
\hline $\begin{array}{c}\text { a. REPORT } \\
\text { Unclassified }\end{array}$ & $\begin{array}{c}\text { b. ABSTRACT } \\
\text { Unclassified }\end{array}$ & $\begin{array}{c}\text { c. THIS PAGE } \\
\text { Unclassified }\end{array}$ \\
\hline
\end{tabular}

\begin{tabular}{|c|c|c|} 
17. LIMITATION & $\begin{array}{c}\text { 18. NUMBER } \\
\text { OF ABSTRACT }\end{array}$ & 19 \\
OF PAGES & 60 & \\
\cline { 3 - 3 } & &
\end{tabular}

Standard Form 298 (Rev. 8-98)

Prescribed by ANSI Std. 239.1 